\title{
PERFORMANCE ANALYSIS AND
}

\section{RADIATION DAMAGE ESTIMATION}

OF THE STOPPING TARGET

\section{MONITOR DETECTORS IN MU2E}

A dissertation submitted to the University of Manchester FOR the Degree of Master of ScIence by Research in the Faculty of Science and Engineering

2020

\section{George M Sweetmore}

Department of Physics and Astronomy in the School of Natural Sciences 


\section{Contents}

\begin{tabular}{lr}
\hline Abstract & 8
\end{tabular}

\begin{tabular}{lr}
\hline Declaration & 9
\end{tabular}

\begin{tabular}{ll}
\hline Intellectual Property Statement & 10
\end{tabular}

\begin{tabular}{ll}
\hline Acknowledgements & 11
\end{tabular}

$\begin{array}{lll}1 & \text { Introduction } & 12\end{array}$

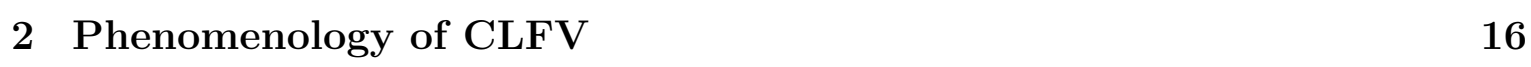

2.1 Fermions in the Standard Model . . . . . . . . . . . . . . . 16

$2.2 \quad$ Majorana Mechanism and Massive Neutrinos $\ldots \ldots \ldots \ldots \ldots$

2.3 Renormalizable Gauge Calculation for a CLFV Process . . . . . . . . . 21

2.4 Model-Independent Effective Lagrangian . . . . . . . . . . . . . . . 24

3 Mu2e: Experimental Overview 27

3.1 Proton Beam . . . . . . . . . . . . . . . . . . . . 30

$3.2 \quad$ Mu2e Solenoids . . . . . . . . . . . . . . . . . . . . . . . . . . . . . . 32

3.2 .1 Production Solenoid $\ldots \ldots \ldots$

3.2 .2 Transport Solenoid . . . . . . . . . . . . . . . . . 34

3.2 .3 Detector Solenoid . . . . . . . . . . . . . . . . . . . . . . 35

3.3 Muon-Stopping Target $\ldots \ldots \ldots \ldots \ldots \ldots$

3.4 Tracker . . . . . . . . . . . . . . . . . . . . . . . . . . 36

$3.5 \quad$ Electromagnetic Calorimeter $\ldots \ldots \ldots \ldots \ldots$

3.6 Cosmic-Ray Veto $\ldots \ldots \ldots \ldots$

3.7 Trigger and Data Acquisition $\ldots \ldots \ldots$ 
4 Stopping Target Monitor 41

4.1 STM Beamline . . . . . . . . . . . . . . . . . . . . . . . . . . . . . . . . 44

$4.1 .1 \quad$ Muon-Beam-Stop Endplug . . . . . . . . . . . . . . . . . . . . . 44

4.1 .2 Instrumentation-Feedthrough-Bulkhead (IFB) Vacuum Window 45

$4.1 .3 \quad$ End Cap Shielding (ECS) . . . . . . . . . . . . . . . . 45

4.1 .4 Cosmic-Ray Veto (CRV) Shielding . . . . . . . . . . . . . . 45

$4.1 .5 \quad$ Sweeper Magnet . . . . . . . . . . . . . . . . . . . . 46

4.1 .6 Field-of-View Collimator . . . . . . . . . . . . . . . . . . . . 46

$4.1 .7 \quad$ Spot-Size Collimator and Shielding House . . . . . . . . . . . . 48

4.2 Stopping Target Monitor (STM) Detectors . . . . . . . . . . . . . . . . 48

$4.2 .1 \quad$ Crystal Choice $\ldots \ldots \ldots$. . . . . . . . . . . . . . . . . . . 49

5 STM Detector Simulation Studies in Mu2e Offline 54

5.1 Radiation Damage to HPGe . . . . . . . . . . . . . . . . . . . . . . 54

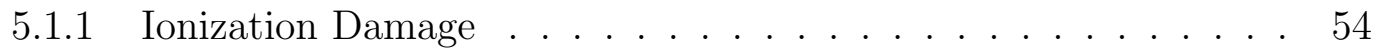

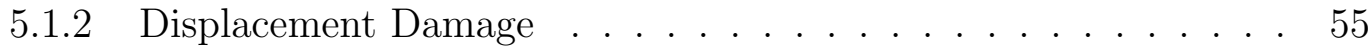

5.2 Mu2e Offline Software and Simulation Studies . . . . . . . . . . . . . . 58

$5.2 .1 \quad$ Estimation of Neutron-Induced Radiation Damage. . . . . . . . 58

5.2 .2 Shielding Effects of Spot-Size Collimator for

Fast Neutrons . . . . . . . . . . . . . . . . . . . . . . . . . . . . 69

$5.2 .3 \quad$ Estimation of Positron-Induced Radiation Damage . . . . . . . 73

$\begin{array}{lll}6 & \text { Conclusion } & 76\end{array}$

\begin{tabular}{lr}
\hline Bibliography & 78
\end{tabular}

Word count 12852 


\section{List of Tables}

5.1 Values of parameters calculated for the Gaussian functions used in fourGaussian resampling scheme . . . . . . . . . . . . . . . 68 


\section{List of Figures}

1.1 Overview of the limits set by experimental $\mu \rightarrow e$ CLFV searches including predicted limits for future experiments [8]. . . . . . . . . . . 15

2.1 Generic Feynman diagram for $\mu \rightarrow e \gamma$ where the loop can include wouldbe Goldstones in renormalizable gauges [24]. . . . . . . . . . . . . . . 21

2.2 Generic Feynman diagrams for the CLFV $\mu \rightarrow e$ process. The top row represents the photonic diagrams (SUSY, Heavy- $\nu$, 2-Higgs-Doublet).

The bottom row represents the contact diagrams (Compositeness, Leptoquarks, Anomalous-couplings). . . . . . . . . . . . . 26

$3.1 \quad$ Energy spectrum of electrons produced via DIO muon decays (red) compared to those produced via free Michel muon decay (blue) [43]. . . . . 29

3.2 Layout of the Fermilab accelerator complex and relative position of the Mu2e facility [4]. . . . . . . . . . . . . . . . . . 30

$3.3 \quad$ Principal design and operation of the three harmonic AC dipole/collimator system [41]. . . . . . . . . . . . . . . . . . . . . . . . 32

3.4 Cross-section design of the Mu2e apparatus (CRV not shown) [41]. . . . 32

3.5 Cross-section design of the Mu2e production solenoid [41]. . . . . . . . 33

3.6 Cross-section design of the Mu2e transport solenoid [41]. . . . . . . . . 34

3.7 Cross-section design of the Mu2e detector solenoid [41]. . . . . . . . . 35

3.8 Muon-beam momentum spectrum compared with the spectrum of stopped muons [41]. $\ldots \ldots \ldots \ldots$

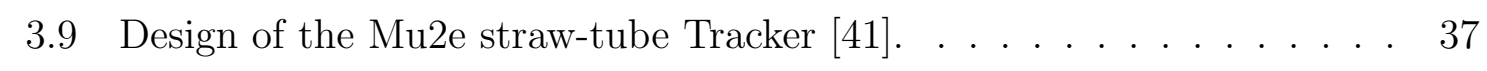

3.10 CAD drawing of the Mu2e electromagnetic calorimeter [43]. . . . . . . 38 
4.1 Data from the AlCap experiment, detailing the prominent, $347 \mathrm{keV}$, $844 \mathrm{keV}$ and $1809 \mathrm{keV}$ peaks respectively [50]. . . . . . . . . . . . . . 44

4.2 Geant4 geometry design detailing the muon-beam stop (MBS), instrumentfeedthrough-bulkhead (IFB) and the end-cap shielding [49]. . . . . . . . 45

$4.3 \quad$ Geant4 geometry design detailing the CRV-shielding and sweeper magnet $[49]$.

4.4 Geant4 geometry design detailing the field-of-view collimator [49]. . . . 47

4.5 Line-of-sight requirements for the field-of-view collimator to ensure full t view of stopping target [53]. . . . . . . . . . . . . . 47

$4.6 \quad$ Geant4 geometry design detailing the spot-size (SS) collimator and detector positions (shielding house not shown) [49]. . . . . . . . . . . 48

4.7 Linear absorption coefficients versus gamma-ray energy for Ge [57]. . . 50

4.8 Efficiency-Energy curve comparison of the Low-Energy Photon Spectrometer (LEPS) (GLP planar), GEM (p-type coaxial) and GMX (ntype coaxial, shown on the right) crystal detectors [56]. . . . . . . . . 52

4.9 Resolution spectra comparison of $\mathrm{NaI}(\mathrm{Tl}), \mathrm{LaBr} 3(\mathrm{Ce})$ and HPGe [61]. . 53

5.1 Diagram of the BNL HPGe detector setup in the irradiation area [63]. .55

5.2 Diagram to show displacement effects in a crystal lattice (a) energetic \begin{tabular}{|c|}
\hline particle creates a Frenkel pair (b) vacancy migrates throughout the \\
\hline
\end{tabular} lattice [65]. . . . . . . . . . . . . . . . . . . . 56

5.3 Geant4 geometry in Mu2e Offline with the Virtual Detectors VD115 and VD101 shown in green. . . . . . . . . . . . . . . . . 59

$5.4 \quad$ Kinetic energy spectrum for electrons and positrons (top row: left to right) and photons and neutrons (bottom row: left to right) detected at VD115 used as input source for simulations. . . . . . . . . . . . 59

5.5 ORTEC study results of Resolution(FWHM) vs Neutron rate for a conventional p-type coaxial detector and a GMX n-type coaxial detector for neutrons from $\mathrm{a}^{252} \mathrm{Cf}$ source [56]. . . . . . . . . . . . . . 61

5.6 Neutron flux distribution from a bare ${ }^{252} \mathrm{Cf}$ source [64]. . . . . . . . . . 62

\begin{tabular}{|ll|l|l|l|l|}
\hline 5.7 & Extraction of Figure & 5.6 & obtained from [64] & (left) and extraction used \\
\hline
\end{tabular} for calculations (right). . . . . . . . . . . . . . . . . . 63 
5.8 NIEL distribution for several particles as a function of incident energy ${ }^{252} \mathrm{Cf}$ source [64] (left) and extraction of neutron NIEL used for calculations (right). . . . . . . . . . . . . . . . . 63

5.9 Calculated KERMA distribution as a function of energy for the ORTEC ${ }^{252} \mathrm{Cf}$ source radiation test. . . . . . . . . . . . . . . . . . . . . . . 63

5.10 Momentum distribution of primary neutron data with the resampled data plotted in red for 35 events and 42 events respectively, demonstrating the smoothing of the resampling scheme. . . . . . . . . . . . 66

5.11 KERMA as a function of neutron energy from 35 and 42 events resampled respectively using the 2016 resampling scheme'. . . . . . . . . . . 67

5.12 KERMA as a function of neutron energy from 35 and 42 events resampled respectively using four-Gaussian resampling scheme'. . . . . . . . 69

$5.131 \mathrm{MeV}$ neutron flux rate at virtual detectors from G4 particleGun for $\mathcal{O}\left(10^{7}\right)$ and $\mathcal{O}\left(10^{8}\right)$ events respectively. . . . . . . . . . . . . . . 70

$5.141 \mathrm{MeV}$ neutron kinetic energy spectrum at virtual detectors from G4 particleGun for 107 events at VD101 and VD89-90 (Left and right respectively). . . . . . . . . . . . . . . . . . . 71

5.15 High energy neutron flux rate at virtual detectors from G4 particleGun for $\mathcal{O}\left(10^{7}\right)$ events. . . . . . . . . . . . . . . . . . . . . . . . 72

5.16 High energy neutron kinetic energy spectrum at VD101 and VD89-90 (left and right respectively) from G4 particleGun for $\mathcal{O}\left(10^{7}\right)$ events. . . 72

5.17 Kinetic energy spectrum of photons at virtual detectors VD101 and VD89 (left and right respectively) produced by $1 \mathrm{MeV}$ and high-energy neutrons (top and bottom row respectively) from G4 particleGun for $\mathcal{O}\left(10^{7}\right)$ events. . . . . . . . . . . . . . . . . . 74

5.18 Positron extraction and data used for KERMA calculation. . . . . . . . 75 5.19 KERMA distribution as a function of positron energy for Mu2e. . . . . 75 


\section{Abstract}

The Mu2e experiment aims to search for the charged lepton flavour violating (CLFV) process of a coherent, neutrinoless, conversion of a muon into an electron within the proximity of an aluminium nucleus. Mu2e seeks to measure the ratio $\left(R_{\mu e}\right)$ of the rate of this conversion process, relative to that of ordinary muon capture. Mu2e will achieve world-leading sensitivity, improving the current limit of $R_{\mu e}<7 \times 10^{-13}$ (90\% C.L.), set by the SINDRUM-II experiment, by an order of $10^{4}$. This corresponds to a singleevent sensitivity of $R_{\mu e}<2.87 \times 10^{-17}(90 \%$ C.L. $)$. Many beyond Standard Model (BSM) theories require CLFV to occur at a rate accessible by Mu2e. Any observation of CLFV at Mu2e would have profound implications on particle physics.

The Stopping Target Monitor (STM) will be comprised of both a HPGe and a LaBr3 detector which will monitor the signals of photons produced in stopped-muon processes to a required accuracy of $10 \%$. To achieve the desired sensitivity, it is imperative that the detectors perform at their optimal level. The STM detectors are placed within a harsh radiation environment in the form of a high energy 'flash' of gamma radiation as well as a flux of fast neutrons.

Estimations of radiation damage to HPGe in literature is limited. This thesis reports an approach which utilizes the Kinetic-Energy Released in Matter (KERMA) parameter that considers the energy dependence of the incident flux and the cross-section information for the incident particle. Resampling schemes have been adopted to give a realistic rate over the experiment run time. The results have then been normalized to the radiation damage caused by a ${ }^{252} \mathrm{Cf}$ source.

The estimated time before annealing of the detector is necessary is found to be 75 and 78 months in the case of neutron damage and $\tau \sim 24$ years, 11 months in the case of electron/positron induced damage. This shows significant improvement on the previous estimations. 


\section{Declaration}

No portion of the work referred to in the dissertation has been submitted in support of an application for another degree or qualification of this or any other university or other institute of learning. 


\section{Intellectual Property Statement}

i. The author of this dissertation (including any appendices and/or schedules to this dissertation) owns certain copyright or related rights in it (the "Copyright") and s/he has given The University of Manchester certain rights to use such Copyright, including for administrative purposes.

ii. Copies of this dissertation, either in full or in extracts and whether in hard or electronic copy, may be made only in accordance with the Copyright, Designs and Patents Act 1988 (as amended) and regulations issued under it or, where appropriate, in accordance with licensing agreements which the University has entered into. This page must form part of any such copies made.

iii. The ownership of certain Copyright, patents, designs, trade marks and other intellectual property (the "Intellectual Property") and any reproductions of copyright works in the dissertation, for example graphs and tables ("Reproductions"), which may be described in this dissertation, may not be owned by the author and may be owned by third parties. Such Intellectual Property and Reproductions cannot and must not be made available for use without the prior written permission of the owner(s) of the relevant Intellectual Property and/or Reproductions.

iv. Further information on the conditions under which disclosure, publication and commercialisation of this dissertation, the Copyright and any Intellectual Property and/or Reproductions described in it may take place is available in the University IP Policy (see http://documents.manchester.ac.uk/DocuInfo.aspx?DocID=487), in any relevant Dissertation restriction declarations deposited in the University Library, The University Library's regulations (see http://www.manchester.ac.uk/library/aboutus/regulations) and in The University's Guidance on Presentation of Dissertations. 


\section{Acknowledgements}

I would like to thank my supervisors

Dr. Marco Gersabeck and Dr. Robert Appleby

and my colleague

Dr. Sophie Middleton

for their continued support, assistance and patience throughout the time on my project as well as providing me with the opportunity to undergo research on this project.

I would like to thank the Mu2e Collaboration as a whole for the opportunity to present my research and continued support throughout.

Lastly I would like to thank my family and friends without the support of which I would have not been able to complete the research detailed within this dissertation. 


\section{Chapter 1}

\section{Introduction}

"Who ordered that?" The quip of Nobel laureate Isidor Isaac Rabi and a sentiment held across the scientific community in light of the discovery of the elusive cousin to the electron, a discovery that not only solved the problems faced within the theoretical framework of the electrodynamic model but also the problems faced with Bethe and Heitler's theory of energy loss in the cosmic-ray energy regime. The discovery of the 'mu-meson' later to be renamed the muon came on 30 March 1937 when Neddermeyer and Anderson announced the results of their infamous cloud chamber experiment [2]. The experiment measured the Bethe-Heitler energy loss of cosmic-ray particles passing through a cloud chamber with a $1 \mathrm{~cm}$ platinum plate placed inside and revealed two distinct types of particle. The first type were those that followed the electromagnetic, multiplicative shower process as outlined by Carlson and Oppenheimer [1] and the second type was a new class of so-called penetrating particles to which two solutions were offered.

"(a) that an electron ( + or - ) can possess some property other than its charge and mass which is capable of accounting for the absence of numerous large radiative losses in a heavy element; or (b) that there exist particles of unit charge, but with a mass (which may not have a unique value) larger than that of a normal free electron and much smaller than that of a proton [2]"

These results were then confirmed in April 1937 when Street and Stevenson published 
similar findings [3]. They discovered that particles that did not undergo electromagnetic showers were penetrating over $6 \mathrm{~cm}$ in lead more than the Bethe-Heitler theory permitted for electrons of this momentum. Not only this but the particles also produced significantly less ionization than that of protons. Thus the conclusion was drawn that there must be a particle in cosmic-rays with an intermediate mass between that of the electron and proton. What followed was a series of events that solidified the presence of the muon and pinned down its characteristics such as mass and life-time.

When neutrinos were discovered to oscillate [4] this opened up an entirely new possibility that in the Standard Model, lepton flavour conservation could be violated. As such, Charged Lepton Flavour Violation (CLFV) could occur through oscillations in radiative decays, at loop level, where a charged lepton would decay into a charged lepton of another flavour. In the theoretical framework of the massive neutrino extension to the Standard Model the rates of CLFV are calculated to be much smaller than the rates calculated for many Beyond the Standard Model (BSM) theories thus experimental detection of a CLFV process would be a direct signature of new BSM phsyics [5]. Not only this but these processes also play a crucial role in testing BSM models that deal with leptogenesis [6] and as such could shed light on the matter/antimatter asymmetry which cannot be explained, in full, by the CP-violation of the quark sector described by the CKM matrix.

The muon is an optimal particle to test for these violating processes because the muon has a much longer life-time than the tau lepton, the muon can be produced much more easily through pi-meson decays and finally the muon has, unlike the tau lepton, a mass small enough to disable major decay channels to hadrons. However to fully understand the entire scope of physics behind the CLFV process, all possible $\tau \rightarrow e, \mu$ decays would also need to be explored.

The muon has three major CLFV decay modes [5]. The first is a direct decay $\mu^{+} \rightarrow e^{+} \gamma$ from a muon to a final state electron with the excess muon rest energy being transferred to a final state photon. The second is a decay into three final state electrons $\mu \rightarrow e^{+} e^{-} e^{+}$. Finally, there is the coherent, neutrinoless conversion of a stopped muon to an electron in the field of a nucleus $\mu^{-} N \rightarrow e^{-} N$. Over the years experiments have set stringent limits on the branching ratios for these decay processes. This dissertation 
will focus on the Mu2e experiment based at Fermilab which aims to probe the final decay mode $\mu^{-} N \rightarrow e^{-} N$ and improve on the limits set by its predecessors.

CLFV in $\mu \rightarrow e$ transitions is primarily experimentally studied in fixed target experiments, however CLFV processes can also be probed in collider experiments [7]. The limits set for the CLFV branching ratio for different processes are displayed in Figure 1.1 including past limits as well as predicted future limits [8]. The MEG experiment at The Paul Scherrer Institut (PSI) in Switzerland offers the best current limit on the $\mu^{+} \rightarrow e^{+} \gamma$ branching ratio in which a muon undergoes a two-body final state decay into a positive electron and a photon emitted back-to-back, both carrying energy equivalent to half the rest mass of the muon. The limit as of 2016 is set at $\mathcal{B}\left(\mu^{+} \rightarrow e^{+} \gamma\right)=4.2 \times 10^{-13}(90 \%$ C.L.) [9]. The SINDRUM-II experiment also situated at PSI offers the best current limit on the ratio parameter for the $\mu \rightarrow e$ conversion in the presence of various different nuclei. The limits for these processes are set at $R_{\mu e}(\mathrm{Au})=7 \times 10^{-13}(90 \%$ C.L. $)$ [10], $R_{\mu e}(\mathrm{Ti})=4.3 \times 10^{-12}(90 \%$ C.L. $)$ [11] and, $R_{\mu e}(\mathrm{~Pb})=4.6 \times 10^{-11}(90 \%$ C.L. $)[12]$. 


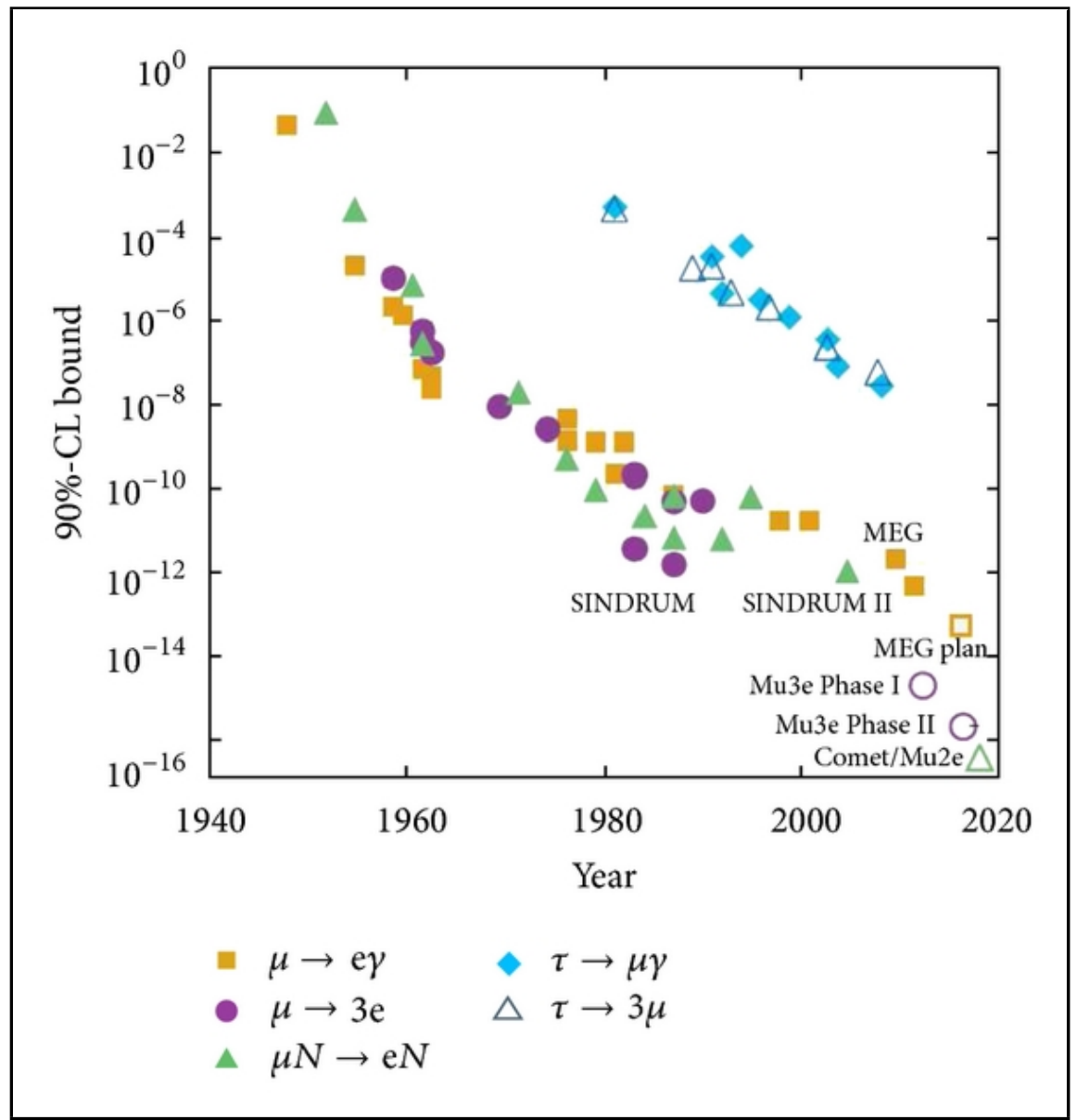

Figure 1.1: Overview of the limits set by experimental $\mu \rightarrow e$ CLFV searches including predicted limits for future experiments [8]. 


\section{Chapter 2}

\section{Phenomenology of CLFV}

Outlined within this chapter is an overview of the phenomenological framework that allows for sizeable rates of CLFV processes to be determined. This is achieved by reviewing the lepton flavour sector of the Standard Model, introducing minimal extensions to the Standard Model to account for neutrino mass, demonstrating renormalisable gauge calculations of the $\mu \rightarrow e \gamma$ CLFV process and, finally, introducing an effective CLFV Lagrangian that accounts for Beyond the Standard Model (BSM) processes.

\subsection{Fermions in the Standard Model}

The Standard Model of electroweak interactions proposed by Glashow, Weinberg and Salam [13] can be used to describe interactions of fermions. The Lagrangian for the electroweak sector takes the form of

$$
\mathcal{L}_{S M}=\mathcal{L}_{g}+\mathcal{L}_{f}+\mathcal{L}_{H}+\mathcal{L}_{Y}
$$

where $g, f, H, Y$ represent the gauge, fermion, Higgs and Yukawa Lagrangian terms, respectively.

To fully understand the lepton sector it is important to highlight the Yukawa Lagrangian term, which describes the fermion mass generation from spontanteous symmetry breaking $(\mathrm{SSB})$ of the electroweak $S U(2)_{L} \times U(1)_{Y}$ symmetry and takes the form 14

$$
\mathcal{L}_{Y}=\Gamma_{m n}^{u} \bar{q}_{m, L} \tilde{\phi} u_{n, R}+\Gamma_{m n}^{d} \bar{q}_{m, L} \phi d_{n, R}+\Gamma_{m n}^{e} \bar{l}_{m, L} \phi e_{n, R}+\Gamma_{m n}^{\nu} \bar{l}_{m, L} \tilde{\phi} \nu_{n, R}+\text { h.c. }
$$


This is summed over the family, indices $m, n$, and $\Gamma_{m n}^{u, d, e, \nu}$ are matrices that describe the so called Yukawa couplings between the Higgs doublet denoted as $\phi$ and the fermions $(\mathrm{u}, \mathrm{d}, \mathrm{e}, \nu$ represent the up quark, down quark, electron and neutrino respectively) and $\mathrm{L} / \mathrm{R}$ represents the chirality of the particle. It is important to note that, as we have $S U(2)_{L}$ singlets from the combinations $\bar{L} \phi R$, that this Lagrangian is of course gauge invariant. It is also important to establish that as the mass terms of the Lagrangian should have no hypercharge $£^{1}$, we must define two separate representations of the Higgs field with hypercharges $Y=+\frac{1}{2}$ and $Y=-\frac{1}{2}$ to allow for mass terms of the down-type quarks and charged leptons and up-type quarks and neutrinos, respectively. In the spinor representation of $S U(2)_{L}$ these fields take the form

$$
\text { for } Y(\phi)=+\frac{1}{2} \text { we have: } \phi=\left(\begin{array}{c}
\phi^{+} \\
\phi^{0}
\end{array}\right)
$$

and we define $\tilde{\phi}_{i}=\epsilon_{i j} \phi_{j}^{*}$, where $\epsilon_{i j}$ is the Levi-Civita symbol, such that

$$
\text { for } Y(\tilde{\phi})=-\frac{1}{2} \text { we have: } \tilde{\phi}=\left(\begin{array}{c}
\phi^{0 *} \\
-\phi^{-}
\end{array}\right)
$$

where $\phi^{+/-}$represents the spin-up and spin-down states respectively. These fields then transform under $S U(2)$ like

$$
\phi_{i} \rightarrow \phi_{i}^{\prime}=U_{i j} \phi_{j}
$$

likewise

$$
\tilde{\phi}_{i} \rightarrow \tilde{\phi}_{i}^{\prime}=U_{i j} \tilde{\phi}_{j}
$$

Now we have these representations for our Higgs fields we can construct mass terms for the fermions using a single Higgs doublet and using $\phi$ and $\tilde{\phi}$, so our Lagrangian now reads

$$
\mathcal{L}_{Y}=f_{\eta} \bar{l}_{L} \phi \eta_{R}+f_{u} \bar{q}_{L} \tilde{\phi} u_{R}+f_{d} \bar{q}_{L} \phi d_{R}+\text { h.c. }
$$

where $\eta=e, \mu, \tau$ and $u, d$ represent up-type and down-type quarks of the same generation respectively.

Now if we choose our fields such that we only include the terms responsible for the fermion mass generation

$$
\phi=\frac{1}{\sqrt{2}}\left(\begin{array}{c}
0 \\
v+h
\end{array}\right) \rightarrow \frac{1}{\sqrt{2}}\left(\begin{array}{l}
0 \\
v
\end{array}\right), \quad \tilde{\phi}=\frac{1}{\sqrt{2}}\left(\begin{array}{l}
v \\
0
\end{array}\right)
$$

\footnotetext{
${ }^{1}$ It is clear to see the hypercharge terms cancel from $Y_{R}+Y_{\phi}+Y_{\bar{L}}=-2+1+1=0[15]$
} 
then our Lagrangian now reads [14]

$$
\mathcal{L}_{Y}=\frac{f_{\eta} v}{\sqrt{2}}\left(\bar{\eta}_{L} \eta_{R}+\bar{\eta}_{R} \eta_{L}\right)+\frac{f_{u} v}{\sqrt{2}}\left(\bar{u}_{L} u_{R}+\bar{u}_{R} u_{L}\right)+\frac{f_{d} v}{\sqrt{2}}\left(\bar{d}_{L} d_{R}+\bar{d}_{R} d_{L}\right)
$$

From this it is easy to see that the mass terms for the fermions can be defined as

$$
m_{i}=-\frac{f_{i} v}{\sqrt{2}}, \quad i=\eta, u, d
$$

Finally, it is important to note that as the Standard Model does not allow for a right-handed counterpart to the neutrino its mass terms simply cannot be due to Yukawa couplings thus the neutrino term in our Lagrangian is non-existent without introduction of right-handed neutrinos and total flavour conservation of leptons can be deduced simply from the minimality of the Lagrangian.

Neutrino mass can be added by a simple Dirac extension to the SSB of the electroweak symmetry in which case the neutrino gets a mass term like other fermions with a Lagrangian [16]

$$
\mathcal{L}_{D}=-\Gamma_{m n}^{\nu} \bar{\nu}_{m, R} \widetilde{\Phi}^{\dagger} L_{n, L}+\text { h.c. } \quad \Longrightarrow \quad\left(m_{\nu}^{D}\right)_{m n}=\frac{v}{\sqrt{2}} \Gamma_{m n}^{\nu}
$$

This extension is only allowed for very small values of $\Gamma^{\nu}$, the maximum of which is $\lesssim 10^{-12}$ to allow for the $\mathcal{O}(\mathrm{eV})$ neutrino mass in accordance with the observed limits. Thus, this model is not ideal and an alternate idea is needed.

\subsection{Majorana Mechanism and Massive Neutrinos}

Ettore Majorana in 1937 [17] posited a mechanism that described particles with real wave equations. This mechanism added new non-renormalisable operators as an extension to the Standard Model [18], which allowed for mass generation of left-handed fermions directly without the necessity of adding a right-handed counterpart. However, this required the fermion to be its own anti-particle in this case. Many theories have since been proposed that suggest the neutrino is indeed a Majorana fermion with a Majorana mass from which the lepton flavour violation would be allowed.

The so called Majorana mechanism is a crucial precursor to the notion of charged lepton flavour violation. Majorana's theory was motivated by the idea of whether it was necessary to describe fermions with equations involving complex numbers. The 
solution to this problem was to construct a new series of Dirac gamma matrices [19] that both satisfied the Clifford algebra and were purely imaginary. These matrices were constructed in the form of tensor products as [20]

$$
\begin{aligned}
& \tilde{\gamma}^{0}=\sigma_{2} \otimes \sigma_{1} \\
& \tilde{\gamma}^{1}=i \sigma_{1} \otimes \nVdash \\
& \tilde{\gamma}^{2}=i \sigma_{3} \otimes \nVdash \\
& \tilde{\gamma}^{3}=i \sigma_{2} \otimes \sigma_{2}
\end{aligned}
$$

where $\sigma_{1,2,3}$ denote the Pauli matrices. From these newly constructed matrices the Dirac equation could be altered to become.

$$
\left(i \widetilde{\gamma}^{\mu} \partial_{\mu}-m\right) \widetilde{\psi}=0
$$

So we can now express our Majorana Lagrangian as:

$$
\mathcal{L}_{M}=-\frac{1}{2} m_{\nu}^{M} \overline{\nu_{L}^{c}} \nu_{L}+\text { h.c. }
$$

From this it is clear to see the feature of the neutrino mass generation requiring it be its own antiparticle. It is important to note that $\nu_{R}$ are complete singlets in the Standard Model and therefore there is no reason that we can't also have $\overline{\nu_{R}^{c}} \nu_{R}$ terms instead of the left-handed terms. Terms of this nature naturally violate total lepton number (L) and can be generated with the addition of dimension-five operators that are compatible with the Standard Model symmetries and are of the form [16]

$$
\mathcal{L} \supset \frac{C_{i j}}{\Lambda}\left(\bar{L}_{m, L}^{c} \sigma_{2} \Phi\right)\left(\Phi^{T} \sigma_{2} L_{n, L}\right)+h . c . \quad \Longrightarrow \quad\left(m_{\nu}^{M}\right)_{m n}=\frac{C_{m n} v^{2}}{\Lambda}
$$

Here $\Lambda$ denotes the mass-scale of the extra degrees of freedom from the newly broken symmetry $\mathrm{L}$ that have been integrated out, $\sigma_{2}$ is the second Pauli matrix and $C_{i j / m n}$ is the associating Wilson coefficient. It follows naturally that in the case where $\Lambda \gg v$ then $\left(m_{\nu}^{M}\right)_{m n} \ll v$.

It is interesting to note that as this requires SSB of the symmetry L from Goldstone's theorem [21] which states that for a Lagrangian with a symmetry group $G$ which can be spontaneously broken to a smaller symmetry group $H \subset G$ that there exists a massless boson for each broken generator of the symmetry group G. This implies that should the neutrino be a Majorana fermion and L is a spontaneously breakable symmetry 
that there should exist a massless Goldstone boson namely a 'Majaron' which could mediate an entirely new class of decays [22].

If we go back to the original Yukawa Lagrangian 2.2 we find that the Yukawa matrices $\left(\Gamma_{f}\right)$ can be diagonalized via unitary rotations such that

$$
\Gamma_{f}=V_{f} \hat{\Gamma}_{f} W_{f}^{\dagger}
$$

The interactions via neutral currents therefore do not induce any sort of flavour violation, however, from the two rotations the charged current interactions of fermions with the $\mathrm{W}$ boson do induce flavour violation and we can therefore construct a chargedcurrent (cc) Lagrangian as follows [16]

$$
\mathcal{L}_{c c}=\frac{g}{\sqrt{2}}\left(\bar{u}_{L} \gamma^{\mu}\left(V_{u}^{\dagger} V_{d}\right) d_{L}+\bar{\nu}_{L} \gamma^{\mu}\left(V_{\nu}^{\dagger} V_{e}\right) e_{L}\right) W_{\mu}^{+}+\text {h.c. }
$$

This clearly shows the flavour violation in the quark sector and assuming the neutrino is massless this results in the flavour conservation of the lepton sector. However, we know the neutrino is not massless and therefore flavour violation in the lepton sector is now possible. This flavour violation caused by massive neutrinos can be described by the Pontecorvo-Maki-Nakagawa-Sakawa (PMNS) matrix described as [23]

$$
U_{P M N S}=\left(V_{e}^{\dagger} V_{\nu}\right)
$$

This matrix can diagonalise the neutrino mass matrix and thus connects the flavour and mass eigenstates for the neutrino as

$$
\nu_{\alpha}=\sum_{k=1,3} U_{\alpha k} \nu_{k}, \quad \alpha=e, \mu, \tau
$$

The PMNS matrix as well as describing neutrino oscillations can also describe CLFV which is forbidden at tree level but can occur through loop diagrams that involve neutrinos and $W$ bosons. 


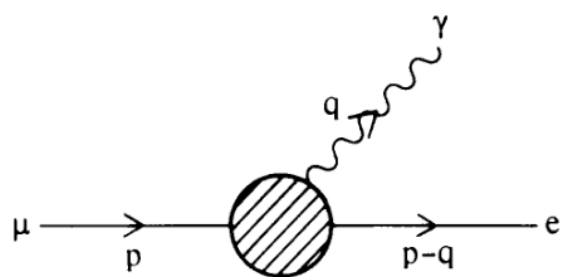

Figure 2.1: Generic Feynman diagram for $\mu \rightarrow e \gamma$ where the loop can include would-be Goldstones in renormalizable gauges [24].

\subsection{Renormalizable Gauge Calculation for a CLFV Process}

It is now important to establish the $R_{\xi}$ gauge calculation for the decay of $\mu \rightarrow e \gamma$ to highlight the calculable, sizeable rate of the CLFV $\mu \rightarrow e$ conversion in minimal extensions to the Standard Model. A detailed account of this calculation can be found in [24], from which the following derivation has been outlined. First it needs to be established that the decay amplitude for the diagram as shown in Figure 2.1 takes the form

$$
T(\mu \rightarrow e \gamma)=\epsilon^{\lambda}\left\langle e\left|J_{\lambda}^{E M}\right| \mu\right\rangle
$$

where, in this notation, $\epsilon^{\lambda}(q)$ denotes the so-called photon polarization and we note that $\mathrm{T}$ has the following Lorentz decomposition where we define $A_{i}(i=1,2, \ldots 6)$ as our invariant amplitudes

$$
\left\langle\mathrm{e}\left|J_{\lambda}^{E M}\right| \mu\right\rangle=\overline{\mathrm{u}}_{\mathrm{e}}(p-q)\left[\mathrm{i} q^{v} \sigma_{\lambda v}\left(A_{1}+A_{2} \gamma_{\mathrm{s}}\right)+\gamma_{\lambda}\left(A_{3}+A_{4} \gamma_{\mathrm{s}}\right)+q_{\lambda}\left(A_{5}+A_{6} \gamma_{\mathrm{s}}\right)\right] \mathrm{u}_{\mu}(p)
$$

Remembering that we have the electromagnetic gauge invariance

$$
\delta^{\lambda} J_{\lambda}^{E M}=0
$$

we find the condition

$$
-m_{\mathrm{e}}\left(A_{3}+A_{4} \gamma_{\mathrm{s}}\right)+m_{\mu}\left(A_{3}-A_{4} \gamma_{\mathrm{s}}\right)+q^{2}\left(A_{5}+A_{6} \gamma_{\mathrm{s}}\right)=0
$$

From which it is found that

$$
A_{3}=A_{4}=0 \text {. }
$$

From this we find that for the on-shell condition where $q^{2}=0$ and thus $\epsilon^{\lambda} q_{\lambda}=0$

$$
T(\mu \rightarrow \mathrm{e} \gamma)=\varepsilon^{2} \overline{\mathrm{u}_{\mathrm{c}}}(p-q)\left[\mathrm{i} q^{v} \sigma_{\lambda v}\left(A_{1}+A_{2} \gamma_{5}\right)\right] \mathrm{u}_{\mu}(p)
$$


If we take the limit where $m_{e}=0$ we find that $A_{1}=A_{2}$ such that the final state electron always be left-handed and using the Gordon decomposition we find that

$$
\begin{aligned}
T & =A_{1} \overline{\mathrm{u}}_{\mathrm{e}}(p-q)\left(1+\gamma_{\mathrm{s}}\right) \mathrm{i} \sigma_{\lambda v} q^{v} \varepsilon^{\lambda} \mathrm{u}_{\mu}(p) \\
& =A_{1} \overline{\mathrm{u}}_{\mathrm{e}}(p-q)\left(1+\gamma_{\mathrm{s}}\right)\left(2 p \cdot \varepsilon-m_{\mu} \gamma \cdot \varepsilon\right) \mathrm{u}_{\mu}(p)
\end{aligned}
$$

such that we only need to factor in the $p \cdot \epsilon$ term into the calculation of our amplitude. In this derivation it is assumed that the neutrinos have the aforementioned Dirac mass, however, this can be extended to the case of Majorana mass [25].

Neglecting contributions from diagrams where the photon vertex is external to the loop $2^{2}$ and by summing over all of the corresponding diagrams, to find the total contribution we find that

$$
A_{1}=A_{2}=e \frac{g^{2}}{8 M^{2}} \frac{m_{\mu}}{32 \pi^{2}} \delta_{v}
$$

Where $\delta_{v}$ is the so called GIM suppression factor that suppresses flavour changing neutral currents (FCNC)

$$
\delta_{v}=\sum_{i} U_{\mathrm{e} i}^{*} U_{\mu i}\left(m_{i}^{2} / M^{2}\right) .
$$

Our dependence on the gauge parameter $\xi$ has now been cancelled which is consistent with the fact that in the limit where $\xi \rightarrow \infty$ (the unitary gauge) the Goldstone bosons decouple.

Thus we obtain for the decay width the expression

$$
\Gamma(\mu \rightarrow \mathrm{e} \gamma)=\frac{m_{\mu}^{3}}{8 \pi}\left(\left|A_{1}\right|^{2}+\left|A_{2}\right|^{2}\right)
$$

Using $\Gamma(\mu \rightarrow e \nu \bar{\nu})=m_{\mu}^{5} G_{F}^{2} / 192 \pi^{3}$ and by defining $G_{\mathrm{F}} / \sqrt{2}=g^{2} / 8 M^{2}$, the branching ratio, of this decay process, as obtained from [24] can be found as

$$
\begin{aligned}
B(\mu \rightarrow \mathrm{e} \gamma) & \equiv \Gamma(\mu \rightarrow \mathrm{e} \gamma) / \Gamma(\mu \rightarrow \mathrm{e} \nu \bar{\nu}) \\
& =\frac{3 \alpha}{32 \pi} \delta_{v}^{2}
\end{aligned}
$$

where $\alpha$ is the fine structure constant. This gives a branching ratio of $\mathcal{O}\left(\sim 10^{-55}\right)$ by utilising the best fit neutrino oscillation parameters taken from [26]

$$
\begin{gathered}
\left|\Delta m_{31(32)}^{2}\right|=2.47(2.46) \times 10^{3} \mathrm{eV}^{2}, \quad \Delta m_{21}^{2}=7.54 \times 10^{5} \mathrm{eV}^{2} \\
\sin ^{2} \theta_{12}=0.307, \quad \sin { }_{13}=0.0241(0.0244), \quad \sin ^{2} \theta_{23}=0.39
\end{gathered}
$$

\footnotetext{
${ }^{2}$ These terms are proportional to $\overline{U_{e}} \gamma U_{\mu}$ and cancel from similar terms from the diagrams where the photon vertex is internal in the loop.
} 
Where, the terms in brackets, correspond to the case where the neutrino mass hierarchy is inverted.

Throughout this derivation total lepton number was assumed to be a global symmetry and thus it has to remain conserved. If instead we extend the $S U(2)_{L} \times U(1)_{Y}$ such that both Dirac and Majorana terms for neutrino mass are included, a new derivation can be formed that is outlined here from a full detailed description to be found in [27]. This hybrid Dirac-Majorana Lagrangian takes the form

$$
\mathcal{L}_{\mathrm{DM}}=\bar{\chi} H \chi
$$

Where we define $\chi$ to be a representation of the six possible self-conjugate fields

$$
\chi=\left(\begin{array}{c}
\nu_{a L}+\left(\nu_{a}^{c}\right)_{L} \\
\nu_{b R}+\left(\nu_{b}^{c}\right)_{R}
\end{array}\right), \quad a, b=e, \mu, \tau
$$

and we define $H$ to be a so-called $6 \times 6$ Majorana mass matrix, which is symmetric

$$
H=\left(\begin{array}{cc}
0 & \frac{1}{2} D \\
\frac{1}{2} D^{T} & \Upsilon
\end{array}\right)
$$

With $D, \Upsilon$ representing the Dirac and Majorana terms respectively. This can be diagonalized by an orthogonal matrix, $W$, such that if we introduce simplifying terms $\Upsilon_{a b}=\Upsilon \delta_{a b}$ and $D_{a b}=D \delta_{a b}$, which don't effect the physics used within the calculation, we obtain $W$ in the form

$$
W=\left(\begin{array}{ll}
U & 0 \\
0 & V
\end{array}\right)\left(\begin{array}{cc}
\hat{C} & \hat{S} \\
-\hat{S} & \hat{C}
\end{array}\right)
$$

Here $U$ and $V$ are orthogonal matrices that represent the diagonalization of the neutrino mass matrix for both chiral states $\left(\nu_{L}, \nu_{R}\right.$ respectively) and $\hat{S}, \hat{C}$ are diagonal matrices such that

$$
S_{i j}=\sin \theta_{i} \delta_{i j}, \quad C_{i j}=\cos \theta_{i} \delta_{i j}, \quad \tan 2 \theta_{i}=D_{i} / \Upsilon
$$

Using this matrix to diagonalise $H$ we find that both chiral states of the neutrino represent superpositions of the six mass-eigenstates $\left(\nu_{i}, N_{i}\right)$ with eigenvalues $\left(m_{i}, M_{i}\right)$, where, $m_{i}$ and $M_{i}$, have values of $\frac{1}{2}\left[\Upsilon \mp\left(\Upsilon^{2}+D_{i}^{2}\right)^{\frac{1}{2}}\right]$ respectively.

Hence the following decay rate for $\mu \rightarrow e \gamma$ is obtained [27]

$$
T_{i}=U_{\mu i} U_{e i} \cos ^{2} \theta_{i} F\left(m_{i}^{2} / M_{w}^{2}\right)+U_{\mu i} U_{e i} \sin ^{2} \theta_{i} F\left(M_{i}^{2} / M_{W}^{2}\right)
$$


Where we define

$$
F(x)=2(x+2) I^{(3)}(x)-2(2 x-1) I^{(2)}(x)+2 x I^{(1)}(x)+1
$$

where

$$
I^{(n)}(x)=\int_{0}^{1} \frac{z^{n}}{[z+(1-z) x]} d z
$$

This introduction of the two eigenstates proposes two distinct solutions. In the first, both eigenvalues are equal $\left(M_{i}\right.$ is also small). This solution allows the usual neutrino oscillations but also allows neutrino-antineutrino oscillations [28]. This results in complete GIM cancellation when summed over the contributing diagrams, as for the case with only Dirac terms and thus has a rate with the same order of magnitude. In the second case where $M_{i} \gg m_{i}$ [29] the GIM cancellation loses its effectiveness. However the mixing between the left-handed chiral states becomes extremely small and thus this process is also highly suppressed.

\subsection{Model-Independent Effective Lagrangian}

CLFV in general becomes very model-dependent in extensions to the Standard Model and thus, following from the maths of [30] [31], it is important to establish a so-called model-independent effective Lagrangian, which provides parameters to reflect the relative size of two distinct types of CLFV models. This effective Lagrangian can be taken in the nucleon regime of mass dimension-six operators as follows

$$
\begin{aligned}
\mathcal{L}_{\mathrm{CLFV}}= & \sum_{f_{q}} \frac{1}{\Lambda^{2}}\left[\lambda_{f}^{S, L} \bar{e} P_{L} \mu+\lambda_{f}^{S, R} \bar{e} P_{R} \mu+\text { h.c. }\right] \bar{q}_{f} q_{f} \\
& +\sum_{f_{q}} \frac{1}{\Lambda^{2}}\left[\lambda_{f}^{V, L} \bar{e} \gamma^{\nu} P_{L} \mu+\lambda_{f}^{V, R} \bar{e} \gamma^{\nu} P_{R} \mu+\text { h.c. }\right] \bar{q}_{f} \gamma_{\nu} q_{f}
\end{aligned}
$$

Here the superscripts $S, V$ represent Scalar and Vector current terms respectively, $L, R$ represent the chiral states, which are determined by $P_{L, R}$, and $f_{q}$ represents all quark flavours from the three generations. Here we have neglected to include contributions from pseudo-scalar and axial-vector terms as these are suppressed in coherent conversions by contributions of the scalar and vector terms. Introducing from here the notation of $q_{l}=u, d, s$ and $q_{h}=c, b, t$ for light and heavy quarks, respectively. We introduce $\varsigma=L, R, \Lambda$ as the energy scale associated with new physics 
contributions $\Lambda \sim 1 \mathrm{TeV}$. Finally we introduce $m_{N}$ as the nucleon mass. Integrating out the heavy quark terms through trace anomaly of the stress-energy tensor [32] we obtain the following Wilson coefficients:

$$
\begin{aligned}
C_{f_{q}}^{S, \varsigma} & =\lambda_{f_{q}}^{S, \varsigma}-\frac{2}{27} \sum_{q_{h}} \frac{m_{f_{q}}}{m_{q_{h}}} \lambda_{q_{h}}^{S, \varsigma} \\
C_{f_{q}}^{V, \varsigma} & =\lambda_{f_{q}}^{V, \varsigma} \\
C_{\Theta}^{\varsigma} & =\frac{2}{27} \sum_{q_{h}} \frac{m_{N}}{m_{q_{h}}} \lambda_{q_{h}}^{S, \varsigma} .
\end{aligned}
$$

If we now introduce the following simplifying terms

$$
\begin{aligned}
F_{q_{l}} & =C_{q_{l}}^{S, L} \bar{e} P_{L} \mu+C_{q_{l}}^{S, R} \bar{P}_{R} \mu+\text { h.c. } \\
F_{q_{l}}^{\nu} & =C_{q_{l}}^{V, L} \bar{e} \gamma^{\nu} P_{L} \mu+C_{q_{l}}^{V, R} \bar{e} \gamma^{\nu} P_{R} \mu+\text { h.c. } \\
F_{\Theta} & =\frac{1}{M_{N}}\left[C_{\Theta}^{L} \bar{e} P_{L} \mu+C_{\Theta}^{R} \bar{e} P_{R} \mu+\text { h.c. }\right]
\end{aligned}
$$

we get an effective Lagrangian of the form

$$
\mathcal{L}_{\mathrm{CLFV}}=\sum_{q_{l}} \frac{1}{\Lambda^{2}} F_{q_{l}} \bar{q}_{l} q_{l}+\sum_{q_{l}} \frac{1}{\Lambda^{2}} F_{q_{l}}^{\nu} \bar{q}_{l} \gamma_{\nu} q_{l}+\frac{1}{\Lambda^{2}} F_{\Theta} \Theta_{\mu}^{\mu} .
$$

It is frequently more useful to represent the effective Lagrangian in a so-called 'lepton only' form [33]

$$
\mathcal{L}_{\mathrm{CLFV}}=\frac{m_{\mu}}{(1+\kappa) \Lambda^{2}} \bar{\mu}_{R} \sigma_{\mu \nu} e_{L} F^{\mu \nu}+\frac{\kappa}{(1+\kappa) \Lambda^{2}} \bar{\mu}_{L} \gamma_{\mu} e_{L}\left(\bar{e} \gamma^{\mu} e\right)+\text { h.c. }
$$

Here we introduce $m_{\mu}$ as the muon mass and $F^{\mu \nu}$ as the photon field strength. The parameter $\kappa$ has been introduced to reflect the relative size of the two operators, the magnetic-moment type operator in the first term and the four-fermion type operator in the second term. The first and second term are often referred to as the photonic and contact terms respectively and represent the contributions from diagrams as shown in Figure 2.2. If $\kappa \ll 1$ the photonic term dominates. If $\kappa \gg 1$ the contact term dominates.

Each of the diagrams shown in Figure 2.2 can have its own model-dependent, phenomenological framework for CLFV processes where:

- SUSY refers to the supersymmetry model. 34.

- Heavy- $\nu$ refers to the existence of a heavy sterile neutrino. 35.

- 2-Higgs-doublet refers to the model where the Higgs boson exists in two doublets 
and can have five physical states: two CP-even, neutral Higgs bosons $h$ and $H(H$ is heavier than $h$ ), the CP-odd pseudoscalar A and two charged Higgs bosons $H^{ \pm}$. [36] - Compositeness refers to the model where the Higgs boson is taken to be of finite size and represents a Nambu-Goldstone field. 37]

- Leptoquarks refers to interactions via the exchange of a so-called leptoquark which are colour-triplet bosons that carry both Lepton and Baryon number. [38]

- Anomalous-couplings refers to the anomalous couplings in three to four gauge boson interactions introduced in operators after EW SSB. 39]

However, these in-depth analyses are beyond the scope of this dissertation and therefore have been omitted. More thorough phenomenological analyses can be found in [33] 40].

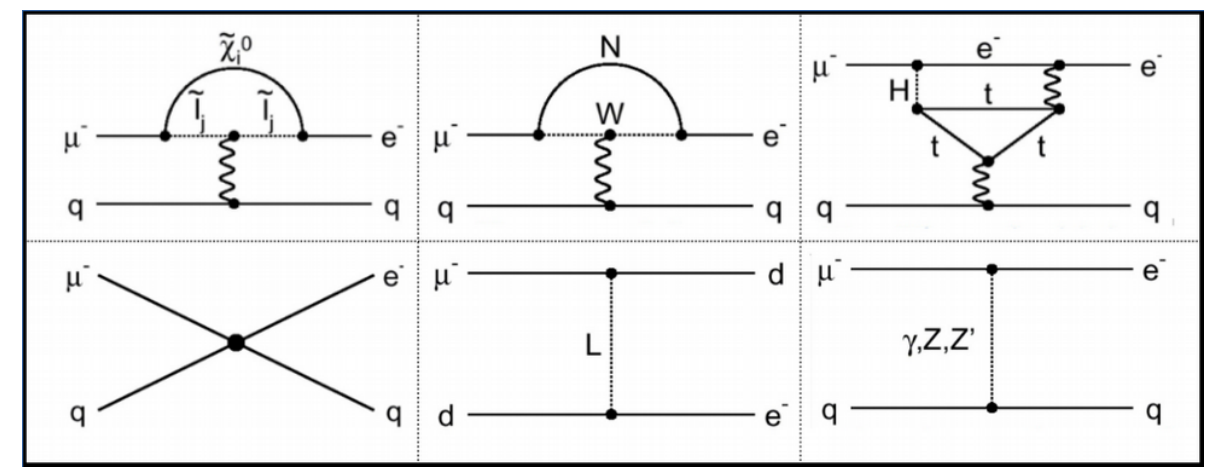

Figure 2.2: Generic Feynman diagrams for the CLFV $\mu \rightarrow e$ process. The top row represents the photonic diagrams (SUSY, Heavy- $\nu$, 2-Higgs-Doublet). The bottom row represents the contact diagrams (Compositeness, Leptoquarks, Anomalous-couplings). 


\section{Chapter 3}

\section{Mu2e: Experimental Overview}

This Chapter provides a brief overview of the Mu2e experiment. A more detailed account can be found in the experiment-specific, technical design report [4]

The Mu2e experiment utilises the distinct process of stopped muon-capture in the field of a target-material nucleus. This process is paramount to probing CLFV effects within and beyond the Standard Model, as once stopped, the muons can cascade to the 1S orbital energy level, where they can undergo the following distinct decay processes. The muon can undergo decay-in-orbit (DIO) which follows the Standard Model decay of $\mu^{-} \rightarrow e^{-} \nu_{\mu} \overline{\nu_{e}}$, or may undergo the process of standard weak nuclear capture $\mu^{-} p \rightarrow \nu_{\mu} n$, however the muon can undergo the CLFV conversion decay process of $\mu^{-} N \rightarrow e^{-} N$. This final process offers a perfect probe of CLFV effects in the Mu2e experiment as the muon rest energy is transferred almost entirely to a monoenergetic electron in the conversion process and thus producing a precise measurable signal.

The binding energy of the muonic atom with the atomic number $Z$ is given by

$$
E_{b} \sim \frac{m_{\mu}^{2} Z^{2} \alpha^{2}}{2}
$$

with $m_{\mu}$ being the muon rest-mass energy. The nuclear recoil energy is given by $E_{\mu}=m_{\mu}-E_{b}$. The nuclear recoil energy the energy of the so-called 'conversion electron' (ce) are related [42] by the expression:

$$
E_{c e}=m_{\mu}-E_{b}-\frac{E_{\mu}^{2}}{2 m_{N}}
$$

where $m_{N}$ is the atomic mass of the target nucleus. This signal electron has an energy of approximately $105 \mathrm{MeV}$. The experiment aims to measure the quantity $R_{\mu e}$ which 
signifies the rate of $\mu \rightarrow e$ CLFV conversion events normalised to the number of stopped muons in the aluminium target. This quantity is defined as:

$$
R_{\mu e}=\frac{\mu^{-}+A(Z, N) \rightarrow e^{-}+A(Z, N)}{\mu^{-}+A(Z, N) \rightarrow v_{\mu}+A(Z-1, N)}
$$

As mentioned previously the current limits for this parameter have been set by Mu2e's predecessor SINDRUM-II at $R_{\mu e}<7 \times 10^{-13}$ (90\% C.L.) [10]. The SINDRUM-II experiment that achieved this rate in 2000 [10] focused a $53 \mathrm{MeV} / \mathrm{c}$ muon beam on a Gold target. During a live time of 75 days, $4.4 \times 10^{13}$ muons were stopped. The electron spectrum was then measured via a spectrometer consisting of several cyclindrical detectors encapsulating the target on the beam-axis which was placed within a $1 \mathrm{~T}$ field superconducting solenoid. Pion contamination was reduced by a factor of $10^{6}$ by directing the beam through a thin moderator to reduce the muon flux. Electrons from the radiative pion capture process within the moderator could reach the target and subsequently scatter into the solid angle of the detector. After removing forward prompt events the measured electron spectrum agreed with the predictions for the spectrum produced via muon decay-in-orbit with no electrons measured in the conversion signal region.

The Mu2e experiment aims to increase the sensitivity of this parameter with a singleevent sensitivity of $R_{\mu e}<2.87 \times 10^{-17}\left(90 \%\right.$ C.L.) [41] which is an order of $10^{4} \mathrm{im}$ provement on the current limit. To achieve this sensitivity Mu2e has to increase the intensity of stopped muons to $1.5 \times 10^{10} \mathrm{~Hz}$ by using a graded solenoidal field. It is also necessary to improve background rejection to ensure conversion only events are selected. There are three distinct backgrounds which can mimic the signal of the conversion electron.

The first background is the DIO process previously mentioned. When the muons undergo this process the nucleus undergoes a coherent recoil, this recoil, in turn, pushes the Michel spectrum endpoint up to the conversion energy peak. Near this conversion endpoint the DIO spectrum rapidly falls as $\left(E-E_{\text {endpoint }}\right)$ [43]. Therefore it is critical that precise momentum measurements are made to enable rejection of the DIO electron background. The spectrum for this process can be seen in Figure 3.1 .

The second major source of background is the possibility of cosmic-ray particles scattering off the target material this, in turn, can produce electrons which mimic the 


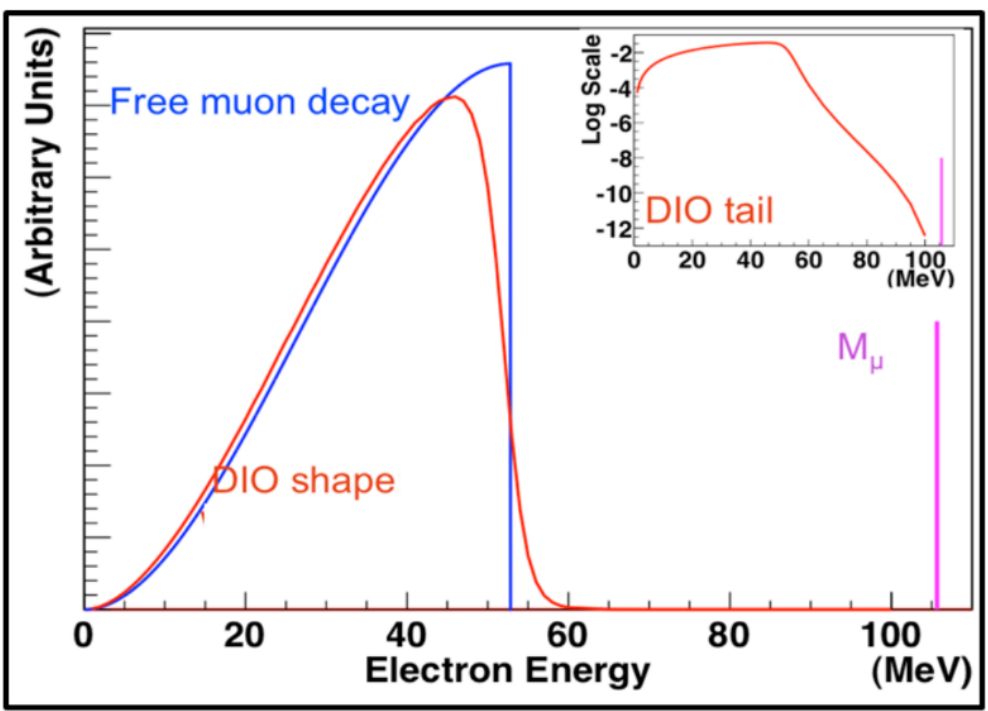

Figure 3.1: Energy spectrum of electrons produced via DIO muon decays (red) compared to those produced via free Michel muon decay (blue) [43].

$105 \mathrm{MeV}$ signal. These backgrounds are indistinguishable from the conversion electrons and therefore it is paramount that the experiment has an active Cosmic Ray Veto (CRV) in place to remove this background contribution as much as possible.

The final major source of background comes from processes in the incident muon beam itself. One example of this is the possibility of pions reaching the stopping target before decaying into muons; these, pions can then undergo a radiative pion capture (RPC) in the target material; this, in turn, creates a gamma which can then experience conversion which can produce a conversion-electron like signal in the detector.

This RPC process provided the strongest limitations on previous muon conversion experiments. Mu2e aims to reduce this background by using a pulsed proton beam to introduce timing cuts which mitigate these backgrounds. 


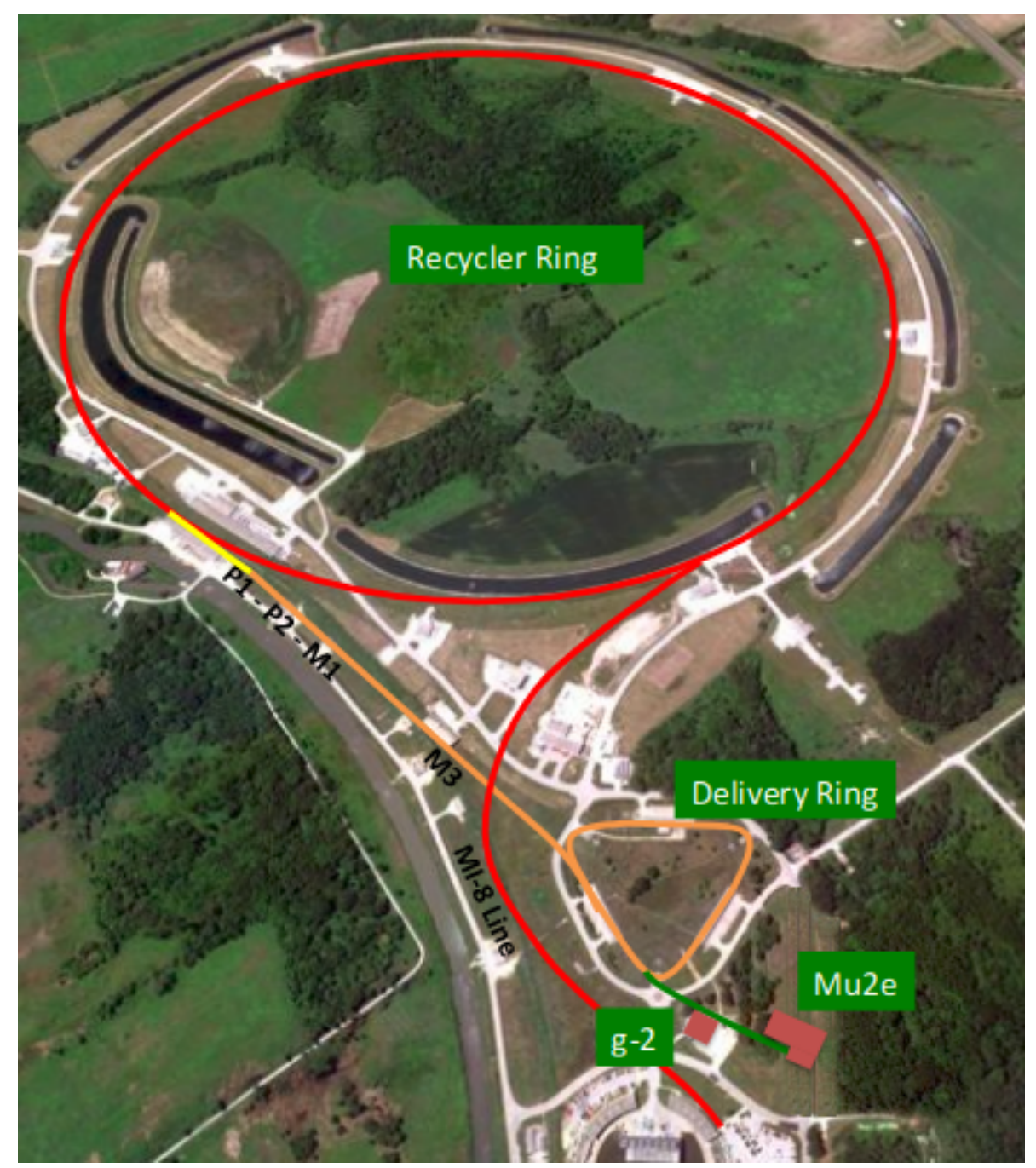

Figure 3.2: Layout of the Fermilab accelerator complex and relative position of the Mu2e facility [41].

\subsection{Proton Beam}

The main feature necessary for the pulsed proton beam is to ensure the secondary muon beam is produced in a time-frame that allows for the stopped muons to undergo the decay processes before the subsequent pulse arrives. The lifetime for stopped muons in aluminium averages to approximately $864 \mathrm{~ns}$ [43. The beam also needs to be of a high enough intensity to produce a high-intensity beam of low-energy muons within this time frame. The proton beam consists of two bunches of $8 \mathrm{GeV}$ protons extracted from the Fermilab Booster ring. These protons are then injected into the Recycler ring where they are allowed to circulate for $90 \mathrm{~ms}$ to allow for an RF sequence to be performed that coalesces the bunches into four $250 \mathrm{MHz}$ bunches which occupy one seventh of the Recycler Ring circumference. These bunches are then injected in 
succession into the Delivery ring. From the delivery ring, the bunches then undergo a resonant extraction where for each turn, a small fraction of the beam is separated and redirected through the extraction beamline. During the resonant extraction the bunch is subjected to an RF knockout technique which allows for fast transverse beam heating and fine control of the spill rate as a feedback tool. This resonant extraction injects the protons into the experiment beamline at a rate of $1695 \mathrm{~ns}$ in accordance with the revolution period of the Delivery Ring.

To suppress the generated background events from beam produced between the successive pulses an extinction system consisting of a high-frequency AC dipole is put in place. This $\mathrm{AC}$ dipole system is based on three distinct harmonics to ensure in-time protons are transmitted to the production target and the out-of-time protons are directed into collimators. The first $300 \mathrm{kHz}$ harmonic is phased to ensure in-time protons pass through the collimator. The second $4.5 \mathrm{MHz}$ harmonic reduces slewing response (the response of an electronic device to a sudden, large change of voltage or current per unit of time.) and ensures minimal loss of in-time beam. Finally a $900 \mathrm{kHz}$ harmonic is used to reduce the maximum amplitude, which, in turn, minimises scraping effects upstream of the collimator. It is important to ensure the extinction system has a high efficiency and, therefore, it is important to optimise the magnet specification. The scaling behaviour of the minimum stored energy within the bending magnets $(U)$ can be defined as

$$
U \propto \frac{1}{\sqrt{\beta_{x} L}}
$$

where $\beta_{x}$ is the so-called beta function that relates to the amplitude and wavelength of a pseudo-harmonic oscillator solution to the Hill equation and $L$ is the magnet length with the assumption of a waist in the non-bending plane [41]. A system of collimators is used to ensure beams outside the admittance are not directed into the transmission of the AC dipole. The general design of this system can be seen in Figure 3.3 .

Once the beam has transversed the extinction system it is then delivered as a beam of $1 \mathrm{~mm}$ transverse radius (RMS) with a duration of $250 \mathrm{~ns}$ to the production target. The production target is a radiatively cooled tungsten rod placed in the evacuated warm bore of a high-field superconducting solenoid. Tungsten is chosen due to its high atomic number and density, its large pion production cross-section and, its ability to radiate the generated heat load without the need of a coolant at a beam power of 

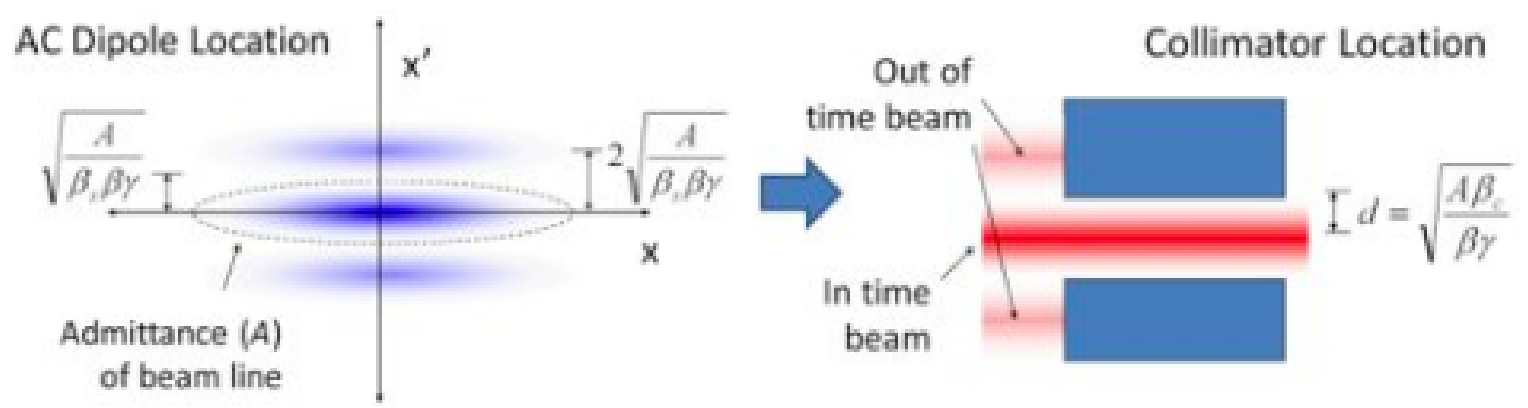

Figure 3.3: Principal design and operation of the three harmonic AC dipole/collimator system [41].

$8 \mathrm{~kW}$.

\subsection{Mu2e Solenoids}

The main beamline of the Mu2e experiment consists of three distinct solenoids as shown in Figure 3.4 the production solenoid, the transport solenoid and the detector solenoid. The production solenoid ensures the proton beam is directed on to the production target and ensures the produced pions are directed to the transport solenoid. The transport solenoid ensures the low-energy negatively charged muon beam is produced and transported to the detector solenoid. The detector solenoid houses the muon-stopping target and all components required to analyze and identify the desired signal and to reduce backgrounds. These solenoids and the subsequent components are discussed in more detail below.

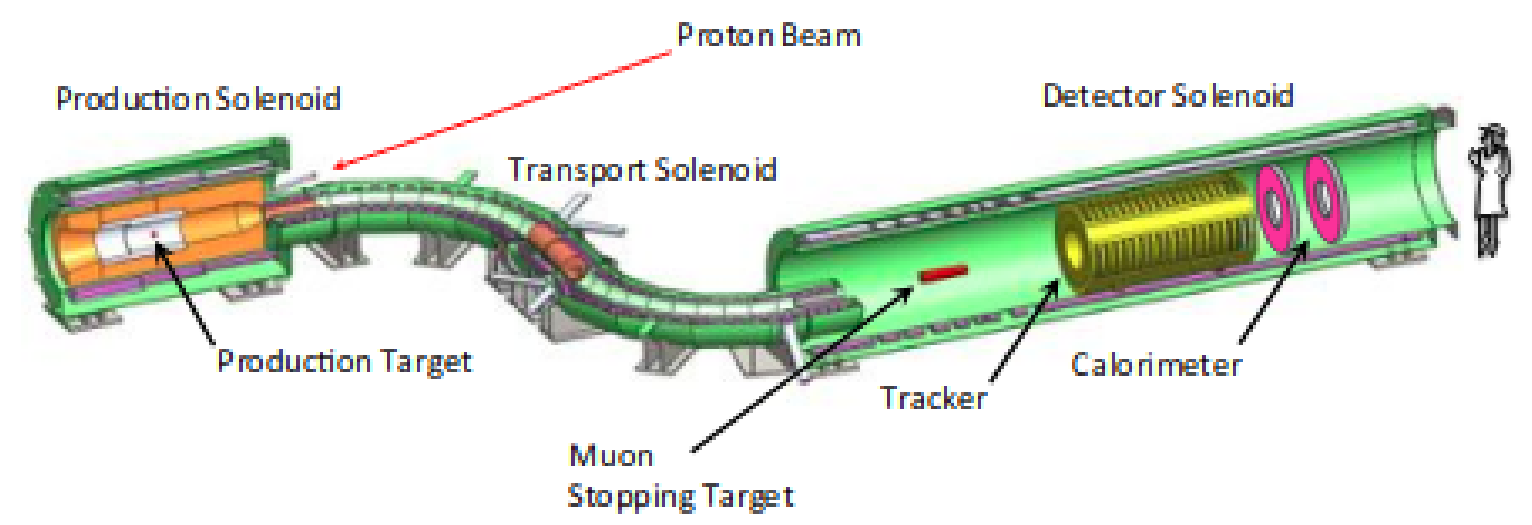

Figure 3.4: Cross-section design of the Mu2e apparatus (CRV not shown) [41]. 


\subsubsection{Production Solenoid}

The production solenoid (PS) as shown in Figure 3.5 is evacuated to $10^{-5}$ Torr and is $4 \mathrm{~m}$ in length with an inner bore diameter of $1.5 \mathrm{~m}$. The solenoid consists of three coils with three, two and two-layer high-current, low-inductance, aluminium-stabilized NbTi axial coils with a decreasing number of windings on each successive coil. These coils form a graded solenoidal field that transitions smoothly from $4.6 \mathrm{~T}$ to $2.5 \mathrm{~T}$. The aluminium stabilizer is used over the alternative copper stabilizer as it can be annealed at room temperature to reverse atomic displacements that cause degradation over time. This atomic displacement is caused primarily by neutrons. This aluminium stabilizer also ensures nuclear heating as a result of the large flux of secondaries is reduced. Finally, aluminium has a smaller density than that of copper thus the overall weight of the solenoid is reduced.

At the centre of the solenoid sits the tungsten production target with a diameter of $12.6 \mathrm{~mm}$ and $16 \mathrm{~cm}$ in length. The protons are injected through a small port at the low-field end where they are directed on to the target producing a large flux of secondary particles. Protons that are not stopped by the target and very-forward going produced secondary particles exit the solenoid through the high-field end. Pions produced at an angle of $\sim 30^{\circ}$ to the solenoid axis in the forward-direction are reflected backwards by the higher-field where they join the backwards-going produced particles and travel helically towards the transport solenoid. The production solenoid warm bore is lined with a bronze heat and radiation shield. This shield limits the damage to the superconductor insulation and aluminium-stabilizer layers and prevents quenching by limiting the heat-load in the cold mass caused by the secondary particle flux.

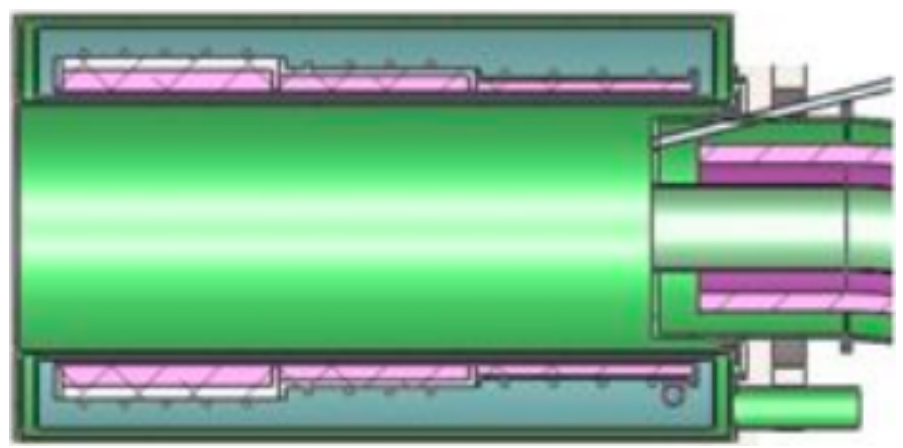

Figure 3.5: Cross-section design of the Mu2e production solenoid [41]. 


\subsubsection{Transport Solenoid}

The S-shaped transport solenoid (TS) as shown in Figure 3.6 is designed to eliminate positively charged particles and line-of-sight neutral particles, as well as high energy, negatively charged particles to ensure the required low-energy negatively charged muon beam is produced and transported to the detector solenoid. This solenoid is designed in five distinct sections: TS1 (1 m straight section), $\mathrm{TS} 2\left(90^{\circ}\right.$ curved section, $3 \mathrm{~m}$ major radius), TS3 (2 $\mathrm{m}$ straight section), TS4 (90 ${ }^{\circ}$ curved section, $3 \mathrm{~m}$ major radius) and TS5 (1 $\mathrm{m}$ straight section). The inner warm bore diameter of the transport solenoid cryostat is $0.5 \mathrm{~m}$. Each section consists of superconducting solenoids and toroids that produce a $2 \mathrm{~T}$ toroidal magnetic field allowing for the separation of negative and positively charged particles drifting in opposite directions perpendicular to the toroidal axis. This magnetic field along with collimators and absorbers along the beamline ensure the necessary elimination of unwanted particles and to ensure selection of the low-momentum muons. To reduce unnecessary transport time the straight sections (TS1, TS3, TS4) have a permanent negative gradient which accelerates the charged particles and eliminates the so-called traps that scatter trapped particles into the detector solenoid late compared to the pulse beam.

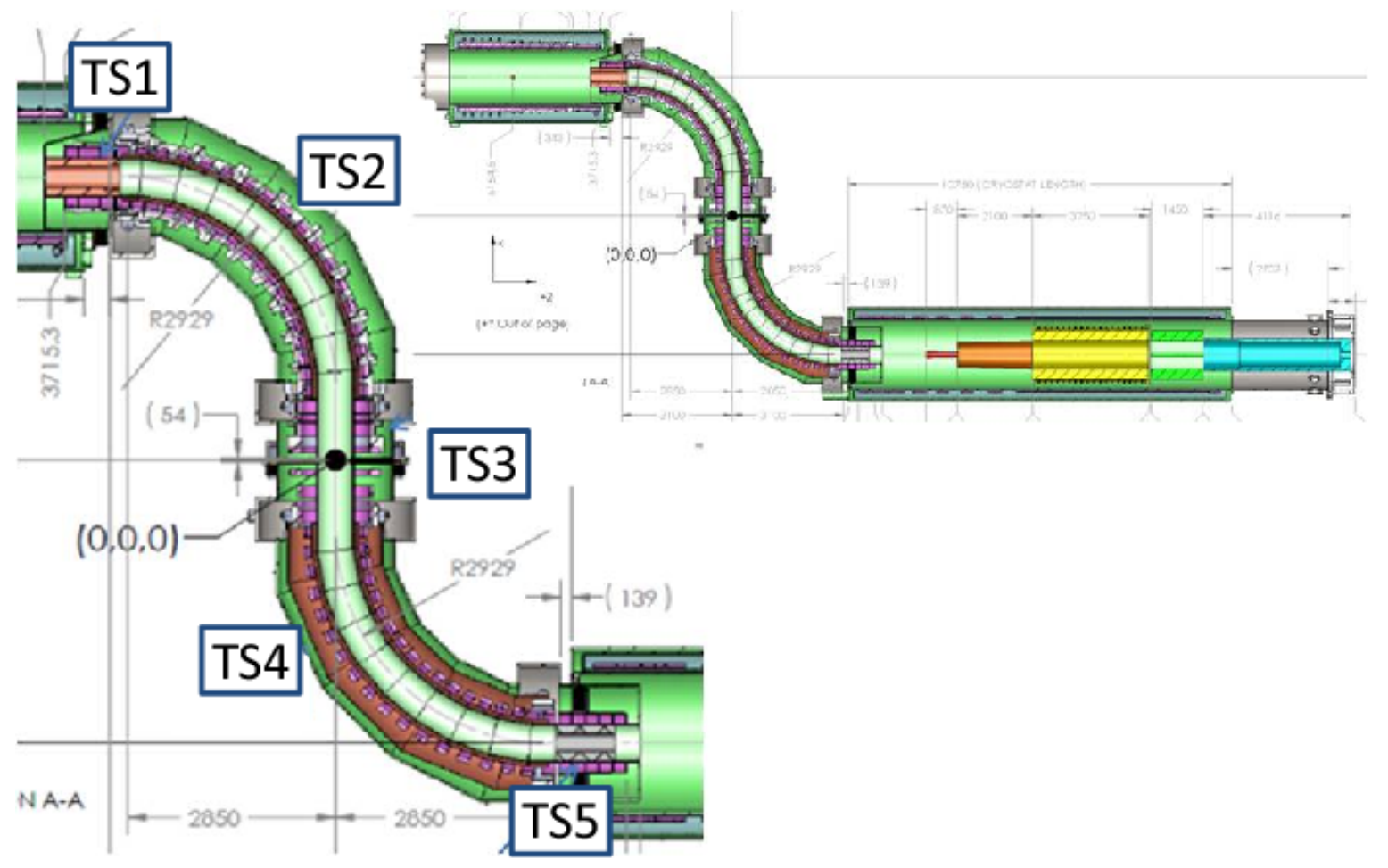

Figure 3.6: Cross-section design of the Mu2e transport solenoid [41]. 


\subsubsection{Detector Solenoid}

The detector solenoid as shown in Figure 3.7 is a low-field magnet of aluminiumstabilized $\mathrm{NbTi}, 11 \mathrm{~m}$ in length with a clear bore diameter of about $2 \mathrm{~m}$ evacuated to $10^{-4}$ Torr. This solenoid houses the muon-stopping target (MST) and the components required to analyze and identify the conversion-electrons as well as the backgrounds including the tracker and calorimeter.

The Muon-Stopping Target is placed within a gradient field of $2 \mathrm{~T}$ to $1 \mathrm{~T}$ which is achieved by introducing spacers which effectively changes the winding density of the superconducting cable. This gradient field ensures back-scattered conversion-electrons are reflected towards the detector components as well as ensuring necessary background reduction of high-energy electrons by incrementally pitch-shifting as they are accelerated which, in turn, separates them from the conversion-electron pitch angle. The detector components are placed within a relatively uniform low-field of $1 \mathrm{~T}$.

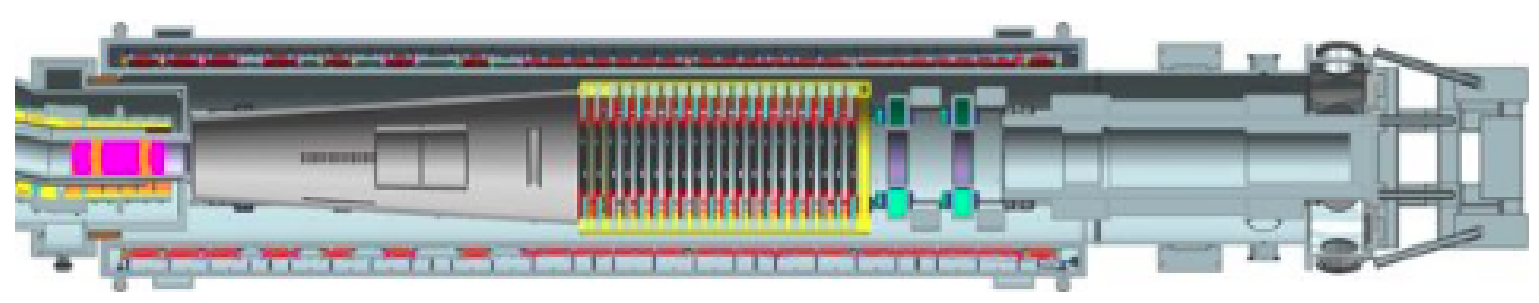

Figure 3.7: Cross-section design of the Mu2e detector solenoid [41].

\subsection{Muon-Stopping Target}

The Muon-Stopping Target consists of seventeen coaxial discs of aluminium, radii varying from 8.33-6.53 $\mathrm{mm}$ down the beamline axis, $0.02 \mathrm{~mm}$ thick, spaced with $50 \mathrm{~mm}$ separation, with a central hole to capture and stop the helically travelling muons. The helical path of the muons is induced by the magnetic field of the solenoid and can be described with an expression for the helix radius $R_{h}$ as follows:

$$
R_{h}=\frac{p_{T}}{q B}
$$

With $p_{T}$ being the transverse momentum, $q$ being the particle's charge and $B$ is the magnetic flux density. 
The stopping target discs are supported by tungsten wires to suppress the DIO background as a material's Z-value determines the DIO spectrum. The DIO endpoint energy decreases for higher Z-value materials which reduces the background; therefore a high Z-value material, such as tungsten, is imperative. The momentum spectrum for the beam muons compared with the spectrum of stopped muons can be seen in Figure 3.8 .

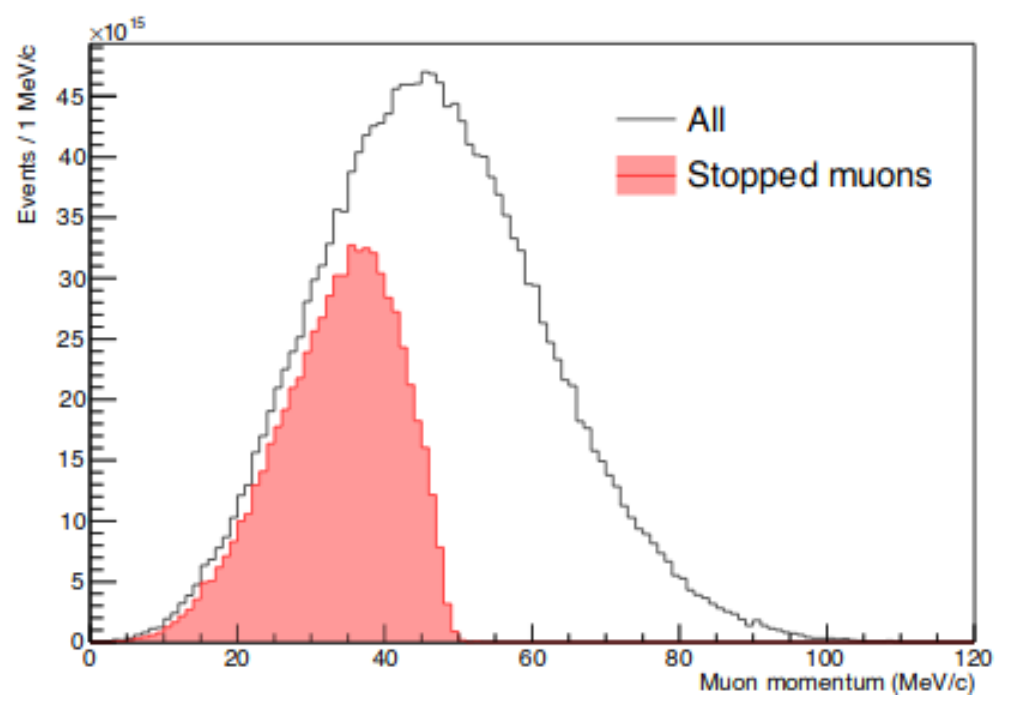

Figure 3.8: Muon-beam momentum spectrum compared with the spectrum of stopped muons [41].

\subsection{Tracker}

The Mu2e tracker provides the high precision momentum measurements necessary to reject the background caused by DIO muons. Muons that capture on aluminium atoms have a $39.1 \%$ chance of undergoing the DIO process in the proximity of the $\mathrm{Al}$ nucleus [44]. The tracker consists of approximately $\sim 20,000,15 \mu$ m metalized Mylar straws in a vacuum, each with a diameter of $5 \mathrm{~mm}$, containing a $25 \mu \mathrm{m}$ sense-wire. The straws are oriented as panels, transverse to the solenoid axis with a $120^{\circ}$ arc. Each panel consists of two staggered layers of 96 straws to help resolve the so-called 'left-right ambiguity' (which side of the sense wire a track passes). A station consists of 12 panels oriented in successive $30^{\circ}$ rotations and the Mu2e tracker consists of 18 consecutive stations with a total length of $\sim 3.2 \mathrm{~m}$ as shown in Figure 3.9. The straws tracker acceptance is limited to a minimum radius of $38 \mathrm{~cm}$; this limit makes the 
tracker blind to the majority of the beam flash and low momentum DIO electrons [43]. Only approximately $3 \%$ of the total DIO rate is observed (electrons of energies greater than $53 \mathrm{MeV}$ ) [41].

The tracker records a digital waveform via ADCs, allowing for $\mathrm{dE} / \mathrm{dx}$ determination to distinguish between electrons and protons that produce highly ionising hits. Transverse and longitudinal hit positions are determined by TDCs and preamplifiers at both ends of the straws to measure the differential drift time. Digitization is performed at the detector, with readout via optical fibres to ensure minimal penetration and a liquid cooling system is in place to ensure optimal operating temperature for the electronics.

The high-side resolution of the tracker is necessary to distinguish between backgrounds and conversion-electrons as the high-side tail would smear background candidates into the signal region. The requirement for the high-side resolution is $\sigma<$ $180 \mathrm{keV}$ [45]. A two-Gaussian sum model of the high-side resolution from simulations determines the core component: $\sigma=115 \mathrm{keV} / \mathrm{c}$, and a significant tail component: $\sigma=176 \mathrm{keV} / \mathrm{c}$ [41]. The low-side resolution is less significant as the tail smears background away from the signal region.

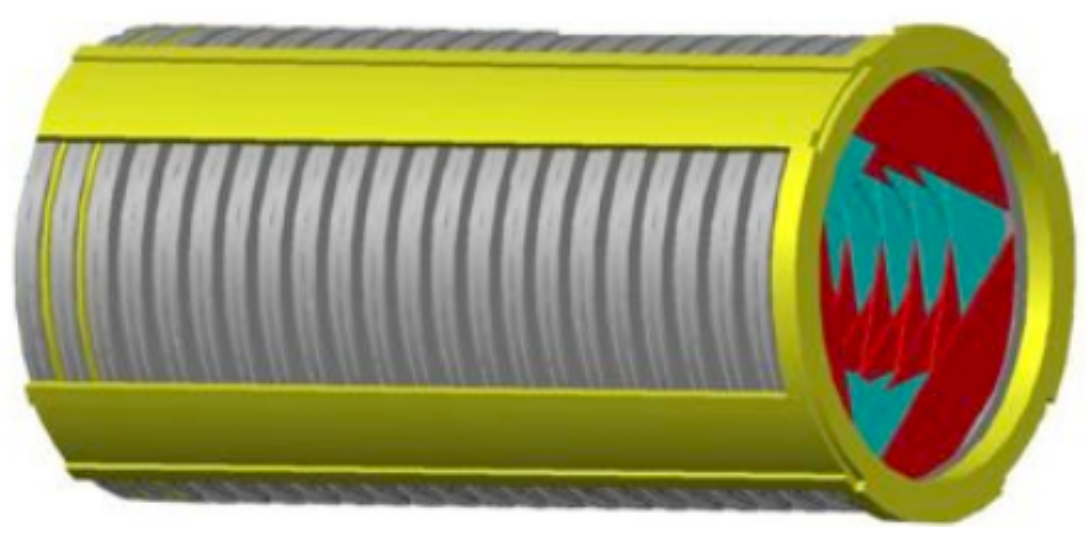

Figure 3.9: Design of the Mu2e straw-tube Tracker [41].

\subsection{Electromagnetic Calorimeter}

The Mu2e Calorimeter as shown in Figure 3.10 consists of 674 undoped CsI scintillating crystals. Each crystal has dimensions of $3.4 \times 3.4 \times 20 \mathrm{~cm}^{3}$ and has a hexagonal shape. 
The calorimeter provides precision energy and time measurements which are critical for particle identification. These precise measurements can help reduce backgrounds from non-vetoed cosmic-ray processes such as trapped muons in the magnetic field or subsequent electrons produced by trapped muons from cosmic rays interacting with the detector material and it can also help to alleviate any misidentification in the tracker due to high hit rates causing the aforementioned high-side tail smearing.

The high precision energy and time measurements are achieved via a complex system of components. Each crystal has a readout via large surface area, solid-state silicon photo-detectors (SiPTs). A laser-flasher system is put in place for each crystal to ensure relative calibration and monitoring is maintained. Absolute calibration is obtained via a circulating, radioactive source, liquid system. To provide the necessary identification of muons and electrons, the calorimeter is required to have an energy resolution of $\sim 5 \%$ and a time resolution of better than 500 ps [43].

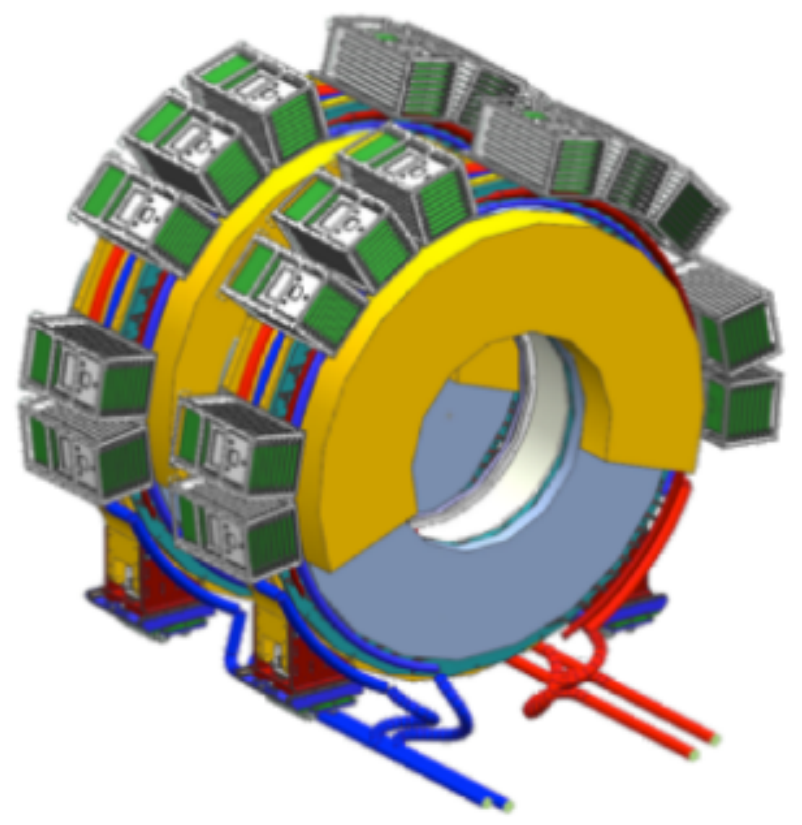

Figure 3.10: CAD drawing of the Mu2e electromagnetic calorimeter [43].

\subsection{Cosmic-Ray Veto}

As mentioned in the previous sections one of the most significant and constant backgrounds comes from processes due to high energy cosmic-ray muons interacting with 
detector material, decaying in flight or subsequently producing electrons and positrons through delta-ray production or as secondary particles which produce $105 \mathrm{MeV}$ conversion like signals. From simulations the estimated amount of cosmic-ray induced background is $0.082 \pm 0.018$ events, assuming a particle identification, muon-rejection factor of 200 and a Cosmic-Ray Veto inefficiency of $10^{-4}$ [41]. Therefore, it is of high importance that the experiment has an active Cosmic-Ray Veto (CRV) system in place to ensure this background is suppressed. The Cosmic-Ray Veto encapsulates the downstream end of the transport solenoid and the entirety of the Detector Solenoid and consists of a shielding wall of concrete as well as a passive shielding detector. The passive shielding consists of four layers of scintillating strips with a $2.0 \mathrm{~cm}$ thickness to ensure a sufficient amount of light to achieve the high light intensity threshold necessary to suppress the backgrounds. The scintillation light is then captured by embedded wavelength-shifting fibres; where, it is subsequently detected by Silicon photomultipliers (SiPMs) at either end of the CRV, neglecting counters placed in close proximity to the transport solenoid downstream region. Between the scintillating layers, an aluminium absorber layer is placed to ensure the reduction of punch-through from the high-energy electrons.

\subsection{Trigger and Data Acquisition}

To ensure necessary efficiency of data acquisition from the Mu2e detectors trigger and data acquisition (TDAQ) subsystems are put in place. The data-acquisition (DAQ) subsystem has an off-detector bandwidth requirement estimated to be $\sim 100$ GBytes/s [46]. The subsystem provides the recording, transferring and filtering (triggers) of the data to ensure necessary rate reduction and data delivery to the offline and online storage systems; where, the data can be further analysed. Readout controllers are used to buffer the beam data from the digitizers during the so-called "live gate" period. The live gate width is nominally the last 1000 ns of each 1695 ns micro-bunch period but it is programmable. The detector will generate an estimated 120 KBytes of zero-suppressed data per micro bunch, for an average data rate of 70 GBytes/sec when beam is present. The calorimeter DAQ system readout produces approximately 25 ADC (analogue-to-digital converter) values (12 bits each) per hit. The cosmic ray 
veto system readout generates approximately 12 bytes for each hit. CRV data is used in the offline reconstruction, so readout is only necessary for timestamps that have passed the tracker and calorimeter filters. The average rate depends on threshold settings. The tracker subsystem readout produces two TDC (time-to-digital converter) values (16 bits each) and typically six ADC values (10 bits each) per hit by reading out each straw at both ends to determine the hit position. The ADC values are the analogue sum from both ends of the straw.

All data filtering and triggering in the Mu2e DAQ architecture is done in software. The production DAQ will use 36 dual-CPU servers. The online processing system must handle a total rate of 192,000 micro-Bunches per second, an average of 5400 events per second per server. The Level-1 trigger is generated when an energy cluster in the calorimeter is detected above a preset energy threshold, the event is then reconstructed in an event builder in a time-ordered manner allowing for background rejection by using space and time information to match the clusters with tracks. The full detailed requirements and operations of the DAQ system can be found in [41]. 


\section{Chapter 4}

\section{Stopping Target Monitor}

Mu2e must achieve a sensitivity to $R_{\mu e}$ greater than that of previous $\mu \rightarrow e$ CLFV searches. Due to the complexity of the processes that deliver the muons from the initial pion decays in the production solenoid to the detector solenoid, a system must be put in place to monitor the rate of negative muons that are stopped in the target material to a precision of $\sim 10 \%$ [47] as well as setting criteria for the fine-tuning of the proton beam. This system is the stopping target monitor (STM). A high-intensity electron beam flash is produced primarily via $\pi^{0}$ production followed by the conversion of decay photons and, also via decays or interactions of beam particles upstream of the stopping target. This beam-flash poses challenges in accurately determining the rate as the electrons can subsequently produce bremstrahlung photons at a rate calculated to be $51 \mathrm{MHz} / \mathrm{cm}^{2}$ with a mean energy of $1.4 \mathrm{MeV}$ [41]. The first possibility to determine the rate of stopped muons is to measure the prompt x-ray photons produced via the orbital transitions of muons that are captured in the atomic orbit of the target material nucleus. Detection of these prompt x-ray photons due to stopped muon capture is not possible with standard solid-state germanium or standard commercial detectors as they are produced within $\mathcal{O}(\mathrm{ps})$ of the initial capture [41] 48] and standard commercial detectors only have timing capabilities of $\mathcal{O}(\mathrm{ns})$ [55]. Due to this timing factor, as well as the high background rate for the prompt x-rays, it is necessary to use a high resolution detector such as a High-Purity Germanium (HPGe) detector.

A second possibility to determine the rate is to instead measure the rate of the semi-prompt photons produced via nuclear capture of the muon,

$$
\mu^{-}+p \rightarrow n+\nu_{\mu}+\gamma
$$


These photons are produced after a time approximate to the muon lifetime in aluminium $(\tau=864 \mathrm{~ns})$. Subsequently, a third, delayed type of $\gamma$-photon can be measured which are produced from activated daughters in the aluminium stopping target due to the aforementioned muonic nuclear capture. These processes have fewer restrictions on timing efficiency of the detector and therefore can be measured more easily compared with the prompt photons; however, the candidate processes for the semi-prompt and delayed production have large error bars on their intensities [49].

There are four major candidates for the normalising rates of the stopped muons [49].

- Prompt: $2 p-1 s, 347 \mathrm{keV}$, x-ray: $79.8(8) \%$ of $\mu$-stops

- Prompt: $3 d-2 p, 66 \mathrm{keV}$, x-ray: $62.5(1.8) \%$ of $\mu$-stops

- Semiprompt: $1.809 \mathrm{MeV}, \gamma$-photon: 51(5)\% of $\mu$-captures

- Delayed: $844 \mathrm{keV}, \gamma$-photon: $10-13 \% \times 71.8 \% \simeq 9.3 \%$ of $\mu$-captures

Due to the high intensity and correspondence with stopped muons the prompt $347 \mathrm{keV}$ is an ideal candidate for detection; however, this candidate has many neighbouring peaks, such as the $3 p-1 s$, and $4 p-1 s$ transitions. Due to the aforementioned highintensity bremstrahlung flash, the detector would suffer a so-called 'dead time'. These factors make HPGe a good candidate for the detector due to the attainable high resolution.

The $66 \mathrm{keV}$ photon is less optimal as it sits in a low-energy regime where rates drop from absorption either within the target material itself or in material between the target and the detector, background rates are also high in this regime and HPGe calibration is difficult.

The Semi-Prompt $1809 \mathrm{keV}$ signal, produced in the ${ }^{27} \mathrm{Al}+\mu^{-} \rightarrow \nu_{\mu}+n+\gamma+{ }^{26} \mathrm{Mg}$ reaction, has been determined to have no significant neighbouring peaks from data acquired at AlCap [50] an experiment designed to determine the photon spectrum from stopped muon processes, based at Paul Scherrer Institut in Switzerland, using the continuous $\mu^{-}$beamline of the $\pi \mathrm{E} 5$ experiment. [51] The lack of neighbouring peaks allows for the use of a detector that can withstand higher rates while having lower resolution such as $\mathrm{LaBr} 3(\mathrm{Ce})$.

The delayed $844 \mathrm{keV}$ peak is unique to the aluminium target and is produced when 
the target material is activated to an excited ${ }^{27} \mathrm{Mg}$ state, this occurs in $17 \%$ of stopped muon captures. This process is outlined as:

$$
{ }^{27} \mathrm{Al}+\mu^{-} \rightarrow{ }^{27} \mathrm{Mg}+\nu_{\mu}
$$

The excited ${ }^{27} \mathrm{Mg}$ subsequently undergoes gamma decay with a lifetime of approximately: $\tau=13.6$ mins producing the $844 \mathrm{keV}$ peak [49]. Stopped muons can also undergo similar processes in nuclei of trapped impurities in the aluminium layer such as oxygen, and therefore, due to the uniqueness of the $\gamma$-photon energy being dependent on the stopped-muon nucleus it is essential for the detector to be of high resolution to resolve the $844 \mathrm{keV}$ peak against the background. This, again, makes HPGe a good candidate [47]. The large lifetime of the beta decaying ${ }^{27} \mathrm{Mg}$ to produce the $844 \mathrm{keV}$ candidate allows the $\gamma$-spectrum to be attained during the idle, beam-off, $1.08 \mathrm{~s}$ period of the pulsed proton beam which is relatively background-free as well as during the beam-on time.

The estimated rate of photons that reach the STM-detector during the so-called extraction period is $\sim 20 / \mathrm{cm}^{2} / \mathrm{s}\left(\sim 90 / \mathrm{cm}^{2} /\right.$ spill-second $)$ with an average energy of $\sim 1.86 \mathrm{MeV}$. Accounting for time-of-flight between the stopping target and the detector, the flash occurs within $~ 200-490$ ns [49]. 

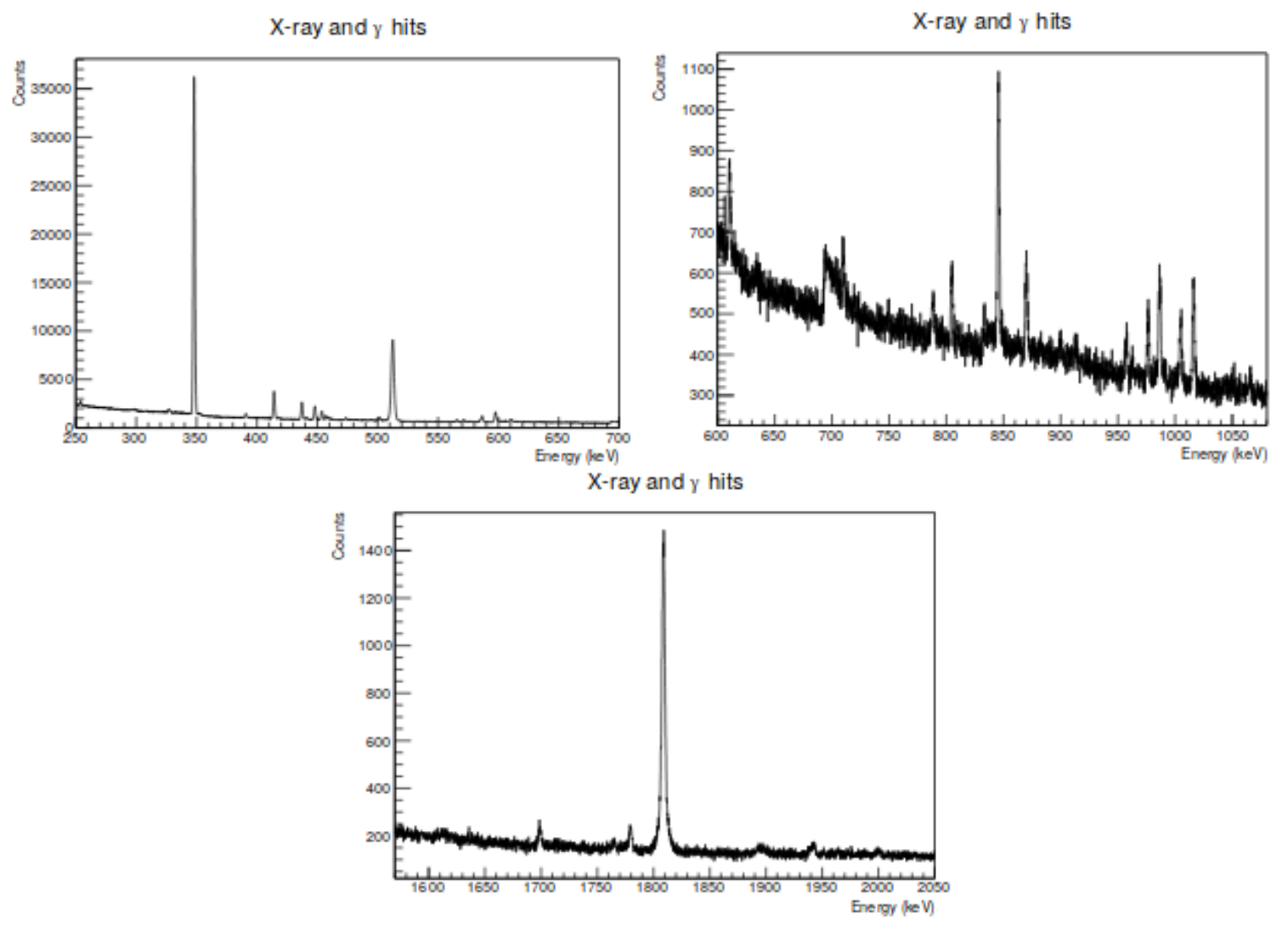

Figure 4.1: Data from the AlCap experiment, detailing the prominent, $347 \mathrm{keV}$, $844 \mathrm{keV}$ and $1809 \mathrm{keV}$ peaks respectively [50].

\subsection{STM Beamline}

To precisely determine the rates of the stopped muon capture, stringent requirements are needed for the detector and beamline components, a detailed account of which can be found in [49].

\subsubsection{Muon-Beam-Stop Endplug}

The requirements for a muon-beam-stop can be found in [52]. The Muon-Beam-Stop component is located down-stream of the calorimeter in the bore of the Detector Solenoid. It is designed such that beam particles and cosmic-ray particles that reach the downstream end of the warm bore are absorbed to minimise the rates of possible backscattered or secondary particles in the tracker and calorimeter. The muon-beamstop requires a $24.0 \mathrm{~cm}$ thick, high-density polyethene (HDPE) endplug with a maximal inner radius of $91 \mathrm{~mm}$ to ensure the x-rays and photons can pass unmoderated from the stopping target to the stopping target monitor detectors. 


\subsubsection{Instrumentation-Feedthrough-Bulkhead (IFB) Vacuum Window}

The instrumentation-feedthrough-bulkhead (IFB) shown in Figure 4.2, is supported at the downstream edge of the muon-beam-stop and is required to have a vacuum window to allow for the transmission of signal photons that ensures maximal beam-background suppression. The recommended material given in [49] for the vacuum window is Mylar with a minimum thickness of $0.212 \mathrm{~mm}$ and a $9.7 \mathrm{~cm}$ radius to maintain the downstream vacuum. The window is located at $\mathrm{z}_{\mathrm{mu} 2 \mathrm{e}}=17321 \mathrm{~mm}$. Simulations have shown that even a $10 \times$ thicker Mylar window will provide the necessary transmission and background suppression [49].

\subsubsection{End Cap Shielding (ECS)}

At the absolute downstream edge of the detector solenoid, a shielded end-cap is placed. The only requirements for this component are to ensure the inner radius exceeds that of the inner radius hole in the Muon-Beam-Stop, such that it accounts for the placement precision, in case the hole is slightly off-axis.

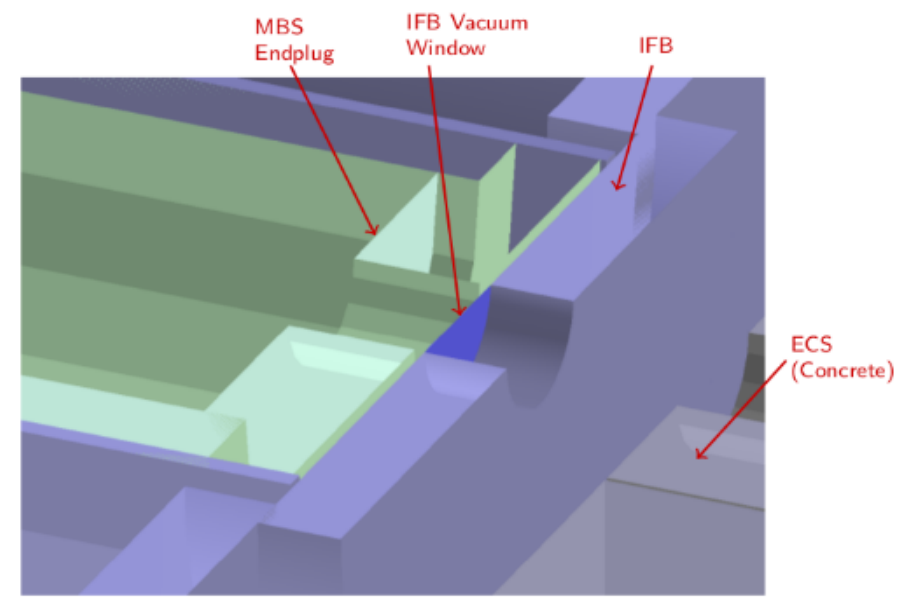

Figure 4.2: Geant4 geometry design detailing the muon-beam stop (MBS), instrumentfeedthrough-bulkhead (IFB) and the end-cap shielding [49].

\subsubsection{Cosmic-Ray Veto (CRV) Shielding}

To allow the necessary rates for the Stopping Target Monitor detectors there cannot be an absorber placed directly downstream of the end-cap. This lack of an absorber 
means muons can escape into the air and can subsequently be stopped within the air itself or in the materials of the downstream components. These stopped muons can then decay into Michel electrons which can cause dead-time in the cosmic-ray veto; therefore, it is necessary to place a wall directly downstream of the endcap and a stainless steel shield surrounding the beamline axis to minimise the possibility of the so-called dead-time. This shielding can be seen in Figure 4.3.

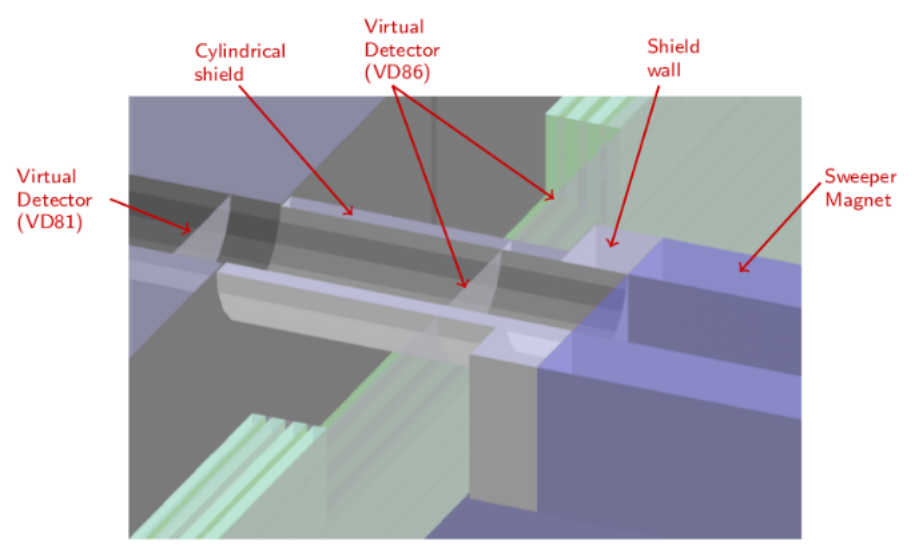

Figure 4.3: Geant4 geometry design detailing the CRV-shielding and sweeper magnet [49].

\subsubsection{Sweeper Magnet}

Charged particles that have escaped beyond the cosmic-ray veto shielding could produce signal like events, reduce the rates of the stopping-target monitor detector as well as causing significant radiation damage. Therefore, a $500 \mathrm{G}$, permanent sweeper magnet (SM), $1.0 \mathrm{~m}$ in length, is placed directly downstream of the cosmic-ray veto stainless steel shield to sweep away the charged particles.

\subsubsection{Field-of-View Collimator}

The stopping-target monitor detectors are required to see only the stopping target at all times. Therefore, it is necessary to block from view all of the aforementioned components downstream from the stopping target. To achieve this a lead collimator is placed directly downstream of the sweeper magnet that is $45.0 \mathrm{~cm}$ in length, $43.2 \mathrm{~cm}$ in width and $45.7 \mathrm{~cm}$ in height, with a collimating aperture that has a radius of $15 \mathrm{~cm}$ in the upstream-most end and $7.8 \mathrm{~cm}$ in the downstream-most end. To suppress any 
signals from muons that have transcended the Sweeper Magnet a $15.0 \mathrm{~cm}(\mathrm{z})$ central region of the collimator is lined with HDPE with a $2.0 \mathrm{~cm}$ thick HDPE absorber at the downstream end of the lining. The maximal inner radius for the collimator is required to be $7.1 \mathrm{~cm}$, to allow for a full view of the stopping target, accounting for misalignments [53]. The design for this collimator can be seen in Figure 4.4 with the line-of-sight requirements shown in Figure 4.5.

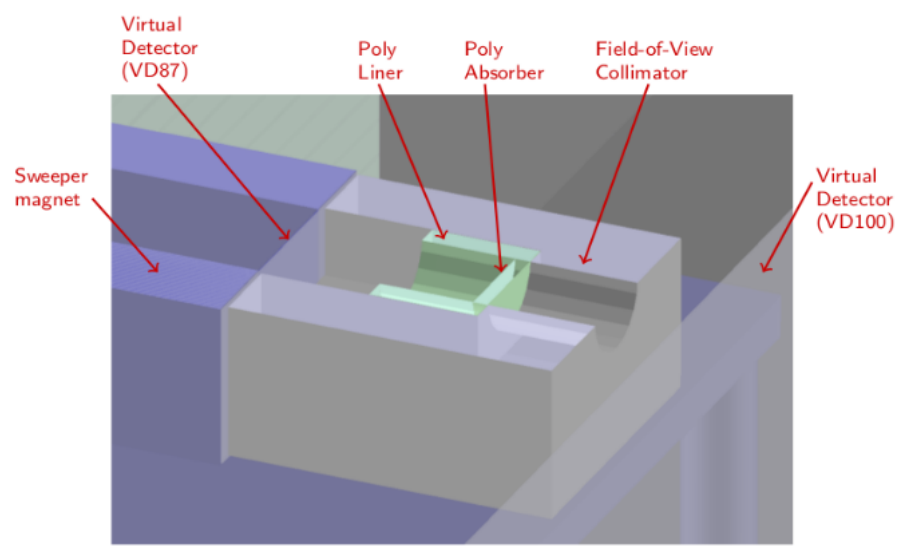

Figure 4.4: Geant4 geometry design detailing the field-of-view collimator [49].
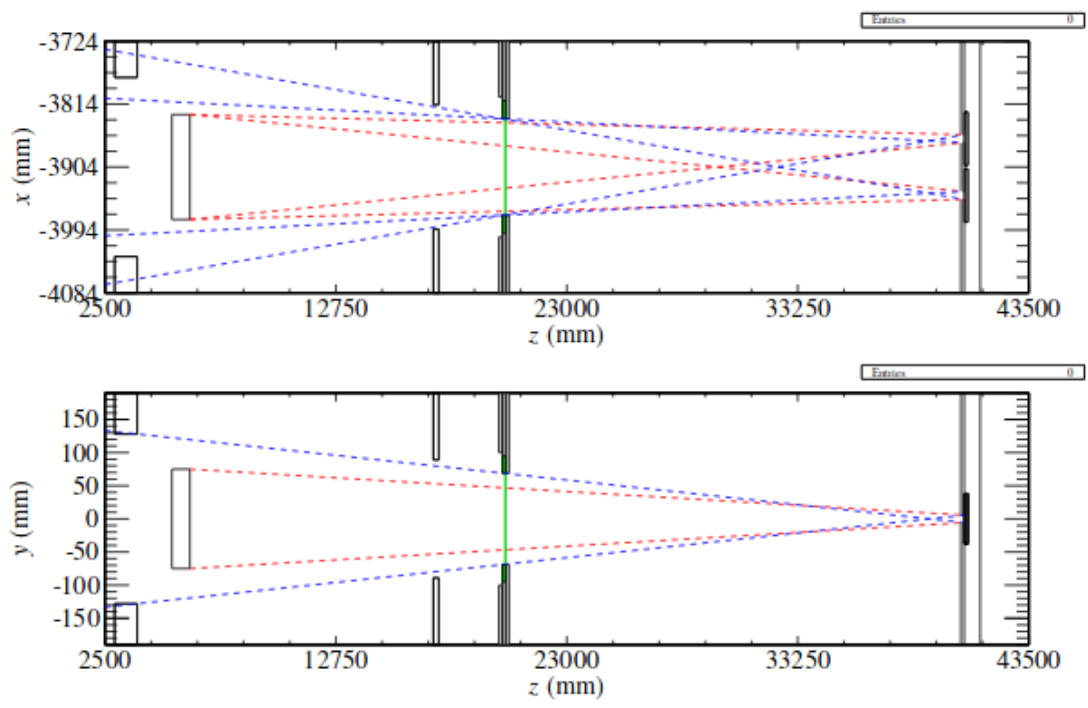

Figure 4.5: Line-of-sight requirements for the field-of-view collimator to ensure full view of stopping target 53 . 


\subsubsection{Spot-Size Collimator and Shielding House}

To ensure the necessary rate reduction for the ttopping target monitor detectors as well as the necessary suppression of any so-called edge-effects from the upstream components, it is necessary to have two collimating apertures of radius $5.642 \mathrm{~mm}$ (to achieve a spot of $\mathrm{A}=1.0 \mathrm{~cm}^{2}$ on the HPGe crystal) directly upstream of the detector crystals. These collimating apertures are drilled within a tungsten block $\sim 20 \mathrm{~m}$ downstream of the field-of-view collimator; that is, $15.24 \mathrm{~cm}$ in length, $43.18 \mathrm{~cm}$ in width and $45.72 \mathrm{~cm}$ in height. $5.08 \mathrm{~cm}$ thick, lead shielding will also be placed above, below and to the sides of the detectors to ensure necessary room background suppression. This is referred to as the shielding house. The design for the spot-size collimator can be seen in Figure 4.6.

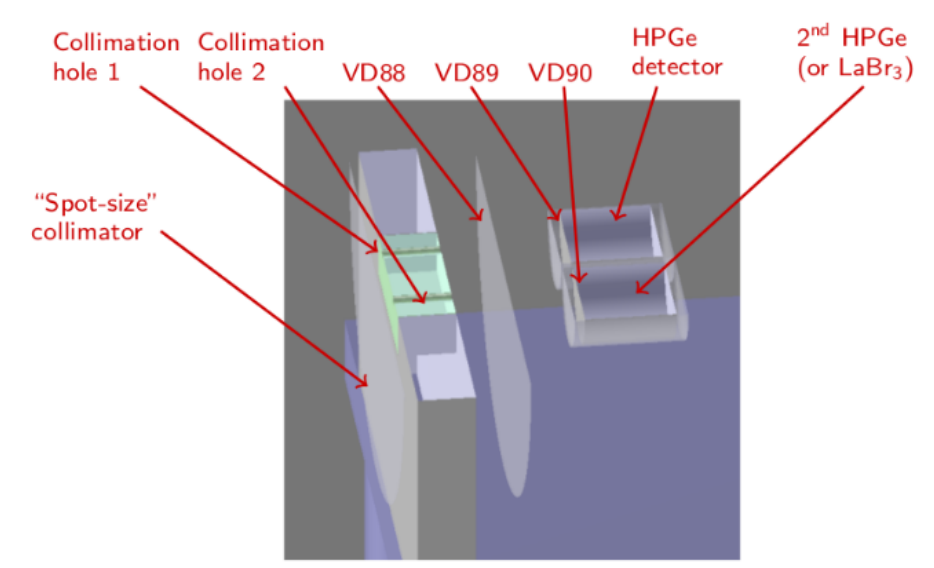

Figure 4.6: Geant4 geometry design detailing the spot-size (SS) collimator and detector positions (shielding house not shown) [49].

\subsection{Stopping Target Monitor (STM) Detectors}

To ensure the rate of stopped muons in the stopping target is measured to the desired $10 \%$ precision it is important that the detectors satisfy the following, stringent, criteria: 54

- The detectors must be able to perform within a high-radiation environment at an optimal rate.

- The detectors must be of high enough energy resolution to resolve any neighbouring background peaks close to the aforementioned normalisation photon peaks. 
- Generally must be capable of minimizing detector acceptance to the large flux of lower-energy charged particles emanating from the stopping target.

- The detectors must suffer minimal dead-time.

- The detector must be able to measure the signals as a function of time, which is required for measuring the $347 \mathrm{keV}$ x-ray photons.

\subsubsection{Crystal Choice}

To achieve the requirements for gamma-ray spectroscopy, a material with a large depletion depth is essential. The depletion depth is defined as the area about the P-N junction in semiconductor materials that is essentially void of charge carriers due to diffusion.

An expression for the depletion depth can be given as [55]

$$
d=\left(\frac{2 \epsilon V}{e N}\right)^{1 / 2}
$$

Where $\epsilon$ is the dielectric constant, $e$ is the electronic charge, $V$ is the reverse bias voltage and $N$ is the net impurity concentration in the bulk semi-conductor material.

To achieve depletion depths of $10 \mathrm{~mm}$ at a fixed voltage of less than $1000 \mathrm{~V}$, the value of the net impurity concentration would need to be reduced to approximately $10^{10}$ atoms $/ \mathrm{cm}^{3}$ (approximately 1 part in $10^{12}$ [55]). Germanium crystals with this level of purity are known as High Purity Germanium (HPGe).

For a HPGe detector to detect a full-energy photon peak all of the photon energy must be deposited in the crystal as a single photoelectric interaction or as multiple events of three possible interaction modes: Compton scattering, pair production or via the photoelectric effect. Due to the fall-off for the photo-electric cross-section as shown in Figure 4.7, the efficiency of detecting photons of energies greater than $200 \mathrm{keV}$ falls off with the total absorption cross-section of Ge [57]. 


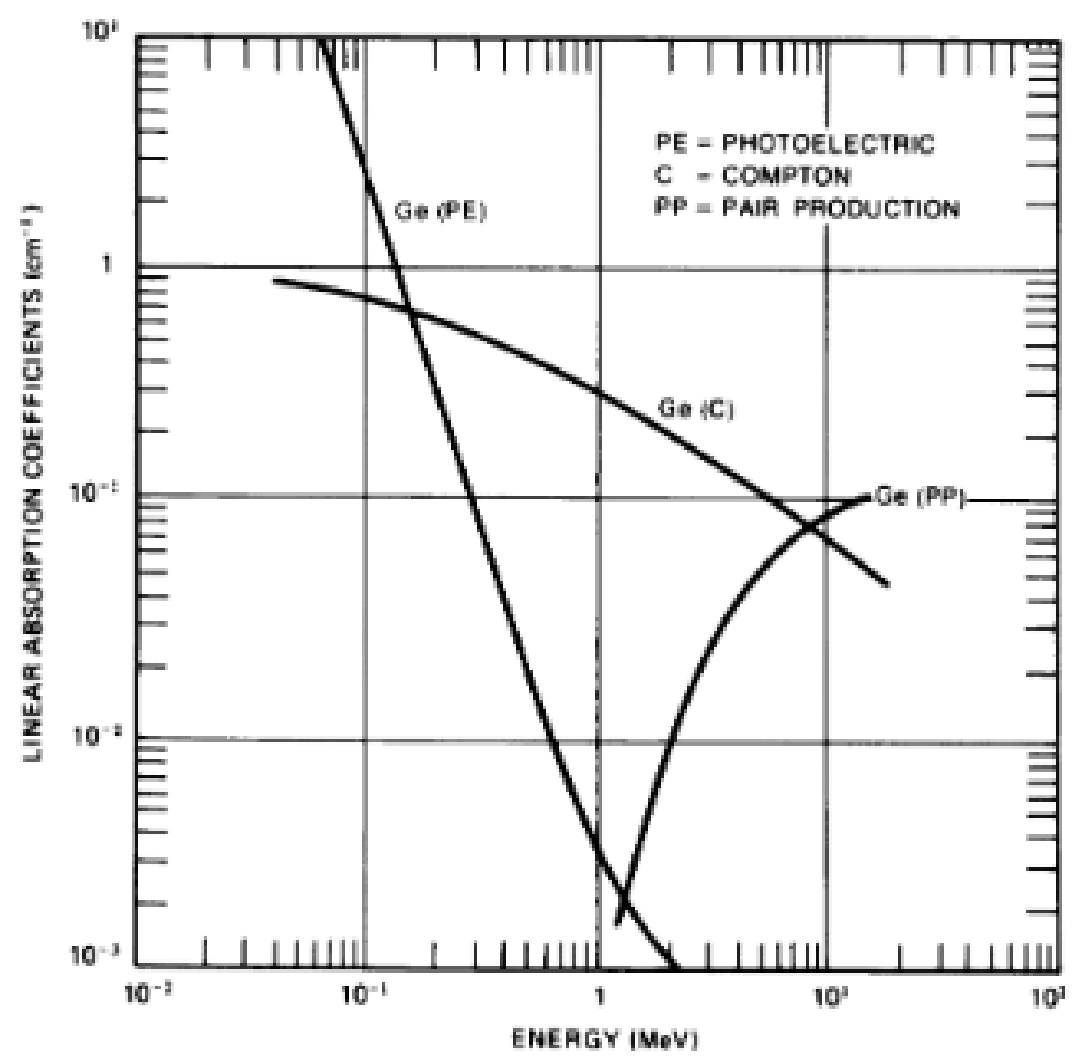

Figure 4.7: Linear absorption coefficients versus gamma-ray energy for Ge [57].

It has been determined, due to the high intensity, high energy neutron environment, that the crystal of choice for the first of the STM detectors would be n-type, coaxial HPGe with an inner $\sim 700 \mu$ m-thick contact of diffused lithium and, an outer, ultra-thin, $0.3 \mu \mathrm{m}$-thick contact of ion-implanted boron [47]. The Ortec product guide for the Gamma-X(GMX) series (coaxial n-type HPGe) lists the following features [56]: Spectroscopy from $3 \mathrm{keV}$ to $10 \mathrm{MeV}$, ULTRA thin boron ion implanted radiation window ideal for Compton Suppression systems, neutron damage resistant (user selfrepair neutron damage option), excellent energy resoluton and peak symmetry, harsh environment (-HE) option, low-background carbon fiber endcap option, PLUS preamplifier option for ultra-high-rate applications and, with huge configuration flexibility including, PopTop, Streamline, and mechanically cooled options.

Due to the harsh radiation environment the detector will be placed in, the necessity for a neutron damage resistant crystal is pivotal; therefore, the n-type HPGe is required. Moreover it can be seen from Figure 4.8 the GMX series detector provides better efficiency at both lower and higher energies. 
The performance for detectors is determined at high energies by its relative efficiency, peak-to-Compton ratio and relative energy resolution. Where the relative efficiency is taken from the method described within the IEEE Standard Test Procedures for Germanium Detectors for Ionizing Radiation [58]. The efficiency is taken at $1.33 \mathrm{MeV}$ relative to the $1.2 \times 10^{-3}$ absolute efficiency of a standard $\mathrm{NaI}(\mathrm{Tl})$ scintillator that is 3 inches in length and diameter placed $25 \mathrm{~cm}$ from a National Institute of Standards ${ }^{60}$ Co source with known intensity. The ${ }^{60}$ Co source is placed $25 \mathrm{~cm}$ from the endcap of the detector and the ratio of the number of counts in the $1.33 \mathrm{MeV}$ photopeak divided by the number of $\gamma$-rays emitted from the source during the same period is taken. This is then divided by the absolute efficiency of the $\mathrm{NaI}(\mathrm{Tl})$ scintillator. The 1.33 MeV peak resolution height is divided by the average Compton plateau between 1.040 MeV and 1.096 MeV to find the peak-to-Compton ratio. The Compton plateau is a result of photons escaping the detector volume due to energy loss via Compton interactions. The product of the absolute resolution and the peak-to-Compton ratio for two detectors of equal dimensions is constant; thus, a detector with a $10 \%$ higher peak-to-Compton ratio has a $10 \%$ better resolution [57]. At low energies, the performance of the detector is determined by its detector window, active surface area and resolution at $5.9 \mathrm{keV}[56]$.

HPGe detectors begin to experience increases in electronic noise and leakage current at temperatures above $\sim 110 \mathrm{~K}$. Temperatures below $\sim 40 \mathrm{~K}$ may introduce trapping effects that can cause deterioration in the energy resolution of the detector. The average energy necessary to create an electron/hole-pair (2.96 eV at $77 \mathrm{~K}$ [57]), varies with temperature at a rate of $2.53 \times 10^{-4} \mathrm{eV}$ per degree $\mathrm{K}$ [59]; therefore, any fluctuations in temperature during measurement would result in peak shifts that would impact the energy resolution. Due to the temperature dependence of the energy resolution of the HPGe detector, it is necessary to cool the detector as close as possible to the optimal $77 \mathrm{~K}$ temperature, using a liquid nitrogen cryogenic system that can be controlled remotely.

The energy resolution as a function of energy for ORTEC detectors is predicted using the following approximate expression [57]

$$
R=\left(N^{2}+2 E\right)^{1 / 2}
$$

where, $\mathrm{R}$ is the full-width at half maximum (FWHM) energy resolution at the desired 
energy peak, $\mathrm{N}$ is the noise-line width and $\mathrm{E}$ is the desired energy (all given in $\mathrm{eV}$ ).

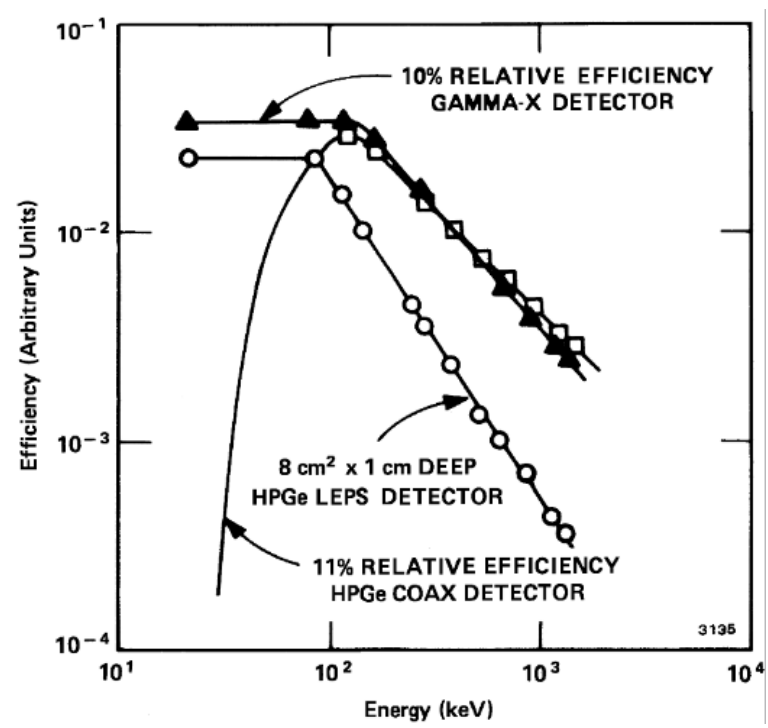

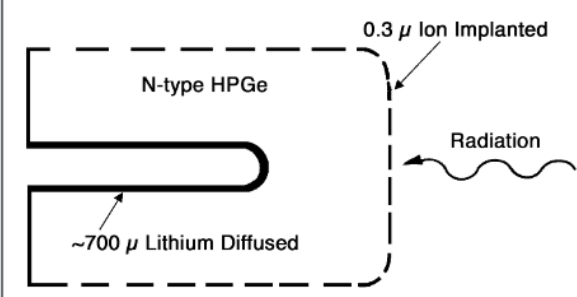

Fig. 2. Configuration of GMX Coaxial Germanium Crystal.

Figure 4.8: Efficiency-Energy curve comparison of the Low-Energy Photon Spectrometer (LEPS) (GLP planar), GEM (p-type coaxial) and GMX (n-type coaxial, shown on the right) crystal detectors [56].

There are two current considerations for the secondary STM detector. The first consideration is a second n-type coaxial HPGe detector, the second consideration is a caesium doped lanthanum bromide $(\mathrm{LaBr} 3(\mathrm{Ce}))$ scintillator. These scintillators do not require cryogenic cooling, are radiation hard, have a timing resolution of $260 \mathrm{ps}$ and have a fast decay time ranging from 15-23 ns and a rise time ranging from 0.7-3.5 ns depending on the concentration of Ce [60].

The LaBr3(Ce) detector has a significantly lower resolution than the HPGe detector as shown in Figure 4.9. This lower-resolution would mean that the lower energy normalisation peaks could not be measured; however, for the $1809 \mathrm{keV}$ it is estimated that a $\sim 2 \%$ resolution could be achieved and as the AlCap data shows no discernible neighbouring peaks at this energy, this peak could be measured with a higher rate in the same time frame as the measure of the conversion signal which could alleviate systematic uncertainties in the measurements [62].

The final consideration for the detectors is the necessity to perform energy and acceptance calibration. To perform these calibrations, Eu-152 and Y-88 sources with calibrated strengths of $\sim 3 \times 10^{5} \mathrm{~Bq}$ (known to $5 \%$ ) will be placed $2 \mathrm{~m}$ upstream of the Spot-Size collimator, which will produce a good calibration of both scales in approximately two hours. These sources have been chosen due to the large span of the 


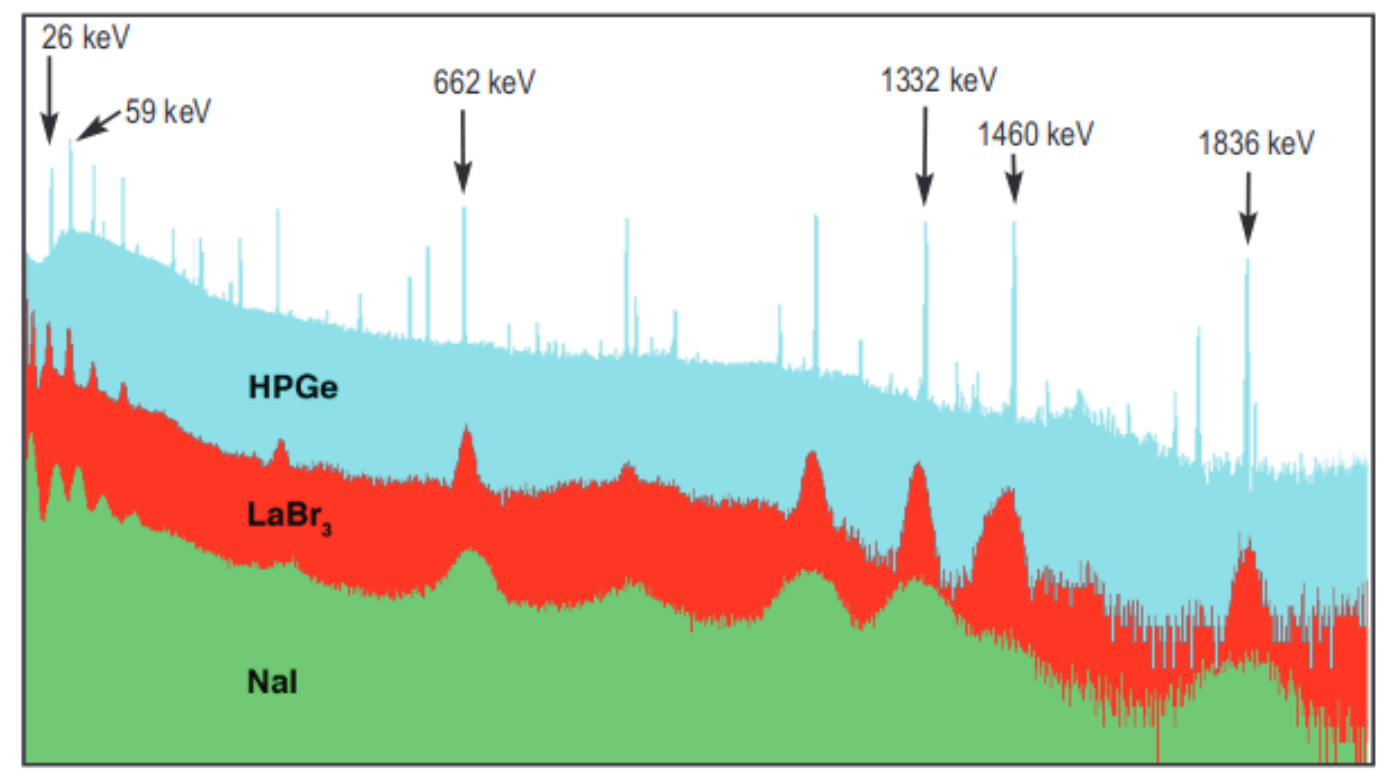

Figure 4.9: Resolution spectra comparison of $\mathrm{NaI}(\mathrm{Tl}), \mathrm{LaBr} 3(\mathrm{Ce})$ and HPGe [61].

$\gamma$-photon spectra produced by the Eu-152 source, as well as the good coverage of the region around the $1809 \mathrm{keV}$ from the Y-88 source. During the data collection period calibration corrections will be performed on the scale of a few hours using the $347 \mathrm{keV}$ $\mathrm{x}$-ray line, $\gamma$-ray lines, and background lines (e.g. the $511 \mathrm{keV}$ line) that emanate from the Stopping Target [49]. 


\section{Chapter 5}

\section{STM Detector Simulation Studies in Mu2e Offline}

\subsection{Radiation Damage to HPGe}

In a high-radiation environment, one has to consider the processes in which particles interact with the detector material and subsequently lead to degradation of the detector quality.

Photons interact with matter via three major processes: Compton scattering, pairproduction and via the photoelectric effect. This leads primarily to possible ionization damage. Charged particles interact primarily with matter via Coulomb scattering. As well as ionization damage this can also lead to subsequent nuclear interactions which can result in displacement damage. Neutrons interact with matter primarily through inelastic and elastic collisions or by transmutation. This also leads to displacement damage. It is therefore useful to separate the radiation damage into the two sub-areas of ionization damage and displacement damage.

\subsubsection{Ionization Damage}

Ionization effects occur when charged particles or high-energy photons interact with matter. The incident particles transfer their energy to the valence electrons within the detector material atoms. This can lead directly to fast electrons, which have been excited across the bandgap into the conduction band, leaving behind a hole. The excited 
electron and hole subsequently lose their excess kinetic energy via lattice scattering. Due to the newly introduced charge separation between the electron-hole pair, if an electric field is present then an electric current is induced. These photocurrents can subsequently lead to a build-up of charge in the detector material which can lead to degradation of performance over time.

A high-radiation test was conducted at Brookhaven National Laboratory (BNL) Solid State Gamma-Ray Irradiation Facility for a p-type, coaxial HPGe detector 663. The detector was placed as close downstream as possible to a 1.5-inch-diameter collimator in front of a ${ }^{60} \mathrm{Co}$ source, shielded by 4.0 inch lead walls as shown in Figure 5.1 . The conclusion of the test was that the detector remained stable after approximately 90hours of exposure with a total of at least $7.5 \mathrm{krad}$ Total-Ionizing-Dose (TID) of gamma radiation.

It was calculated in [64] that by taking the approximate STM rate to be $48 \times 10^{3} \mathrm{MeV} / \mathrm{cm}^{2} / \mathrm{s}$ and by taking $1 \mathrm{krad}=6.66 \times 10^{13} \mathrm{MeV}$, and $3.15 \times 10^{7} \mathrm{~s} /$ year; then, $0.023 \mathrm{krad} /$ year exposure is estimated for the STM detectors. This estimate suggests that the experiment could run for $>\mathcal{O}\left(10^{2}\right)$ years before reaching the $7.5 \mathrm{krad}$ dose deposited in the BNL study.

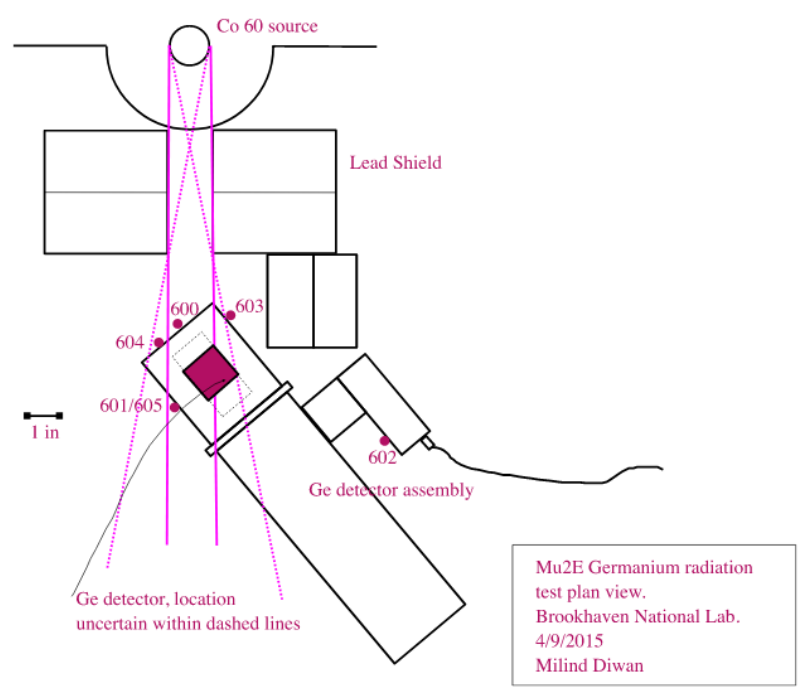

Figure 5.1: Diagram of the BNL HPGe detector setup in the irradiation area 63 .

\subsubsection{Displacement Damage}

The second type damage one has to consider when dealing with crystal detectors in high-radiation environments is displacement damage. 
Displacement damage in ionic lattices of crystals occurs when a fast, high-energy particle interacts with an atom within the lattice, displacing it from its original position. This displaced atom in a non-lattice position is known as an interstitial and the now vacant position the atom had been displaced from is known as a vacancy. The formation of these vacancy-interstitial pairs, known more commonly as Frenkel pairs, has a knock-on effect that can lead to the displacement of further atoms within the crystal lattice or cause ionization [49. The vacancies in the crystal lattice can be interpreted as hole-like such that they are mobile in the lattice structure at room temperature as shown in Figure 5.2 and the presence of such vacancies translates to deep or shallow energy-trap states within the bandgap of the crystal atoms [65].

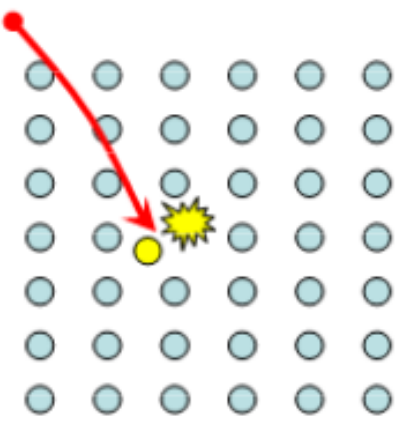

(a)

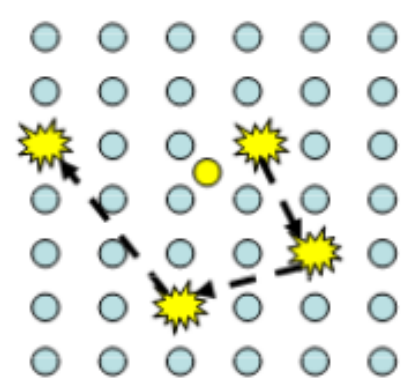

(b)

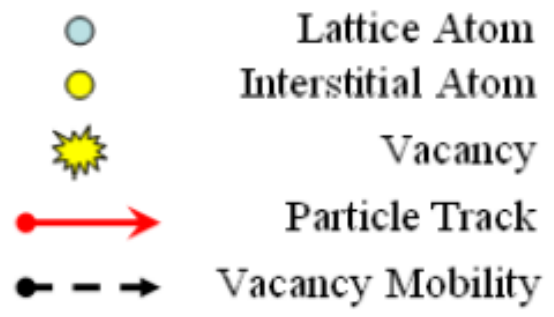

Figure 5.2: Diagram to show displacement effects in a crystal lattice (a) energetic particle creates a Frenkel pair (b) vacancy migrates throughout the lattice 65 .

Building on the model proposed by Kinchin and Pease [66], Norgert, Robinson and Torrens proposed a new model for calculating the number of defects formed within a crystal lattice from an initial primary knock-on atom (PKA) [67]. The model can be taken in the form as given in [68] to be

$$
N_{N R T}=\kappa \frac{\xi(T) T}{2 E_{t h}}
$$

where $N_{N R T}$ is the number of defects, $\kappa=0.8$ is the displacement efficiency, $T$ is the kinetic energy of the PKA, $\xi(T)$ is the Lindhard partition function according to the LSS theory and finally $E_{t h}$ is the displacement damage threshold. $\xi(T)$ takes the form

$$
\xi(T)=\frac{1}{\left.1+F_{L} \cdot 3.4008 \cdot \epsilon(T)^{1 / 6}+0.40244 \cdot \epsilon(T)^{3 / 4}+\epsilon(T)\right)}
$$


with,

$$
\begin{aligned}
& F_{L}=30.724 \cdot Z_{1} \cdot Z_{2} \sqrt{Z_{1}^{2 / 3}+Z_{2}^{2 / 3}} \\
& \epsilon(T)=\frac{T}{0.0793 \frac{Z_{1}^{2 / 3} \cdot \sqrt{Z_{2}}}{\left(Z_{1}^{2 / 3}+Z_{2}^{2 / 3}\right)^{3 / 4}} \cdot \frac{\left(A_{1}+A_{2}\right)^{3 / 2}}{A_{1}^{3 / 2} \sqrt{A_{2}}}}
\end{aligned}
$$

where subscript 1 and 2 represent the projectile and target respectively. We finally obtain the number of defects $v(T)$ to be

$$
\begin{array}{ll}
v(T)=0 & \text { for } 0<T \leq E_{t h} \text { (phonons) } \\
v(T)=1 & \text { for } E_{t h}<T \leq 2 E_{t h} \\
v(T)=T / 2 E_{t h} & \text { for } 2 E_{t h}<T \leq E_{c} \\
v(T)=E_{c} / 2 E_{t h} & \text { for } T>E_{c}
\end{array}
$$

These conditions are obtained, based on the assumptions that: (a) the secondary cascade is initiated by a sequence of two-body elastic collisions between atoms in the lattice; (b) in the collision process, the energy transferred to the lattice is zero; (c) only atomic collisions take place for energies below a so-called cut-off energy $\left(E_{c}\right) ;(\mathrm{d})$ no additional displacement occurs once the cut-off energy is exceeded, (e) the cross section of energy transfer is given by the so-called hard-sphere model.

The partition function $\xi(T)$ becomes important for the displacement damage estimation proposed by Dale et al. [69], by defining a quantity named Non-Ionizing Energy Loss (NIEL). The so-called NIEL gives an estimation that the bulk radiation damage is proportional to the kinetic energy imparted on displaced silicon atoms and is expressed, by convention, relative to the radiation damage caused by a given flux of 1.0 MeV neutrons as formalised in ASTM E722-14 [71]. We find an expression for the NIEL from [70] as

$$
\operatorname{NIEL}(E)=\frac{N_{A}}{A} \int_{T_{\min }}^{T_{\max }} \xi(T) T\left(\frac{d \sigma}{d T}\right)_{E} d T
$$

where, $N_{A}$ is Avagadro's number, $A$ is the atomic mass of the crystal lattice atom, $A_{1}$ is the atomic mass of the projectile, $T_{\max }=4 T_{0} A A_{1} /\left(A+A_{1}\right)^{2}, T_{\min }=2 E_{t h}$ and finally, $\left(\frac{d \sigma}{d T}\right)$ is the differential cross-section for creating a recoil with kinetic energy $\mathrm{T}$. 


\subsection{Mu2e Offline Software and Simulation Studies}

Mu2e Offline is an experiment-specific software package using the Fermilab Scientific Computing Division developed, art $(\gamma)$ [72], event-processing based framework. The Offline software package is based in $\mathrm{C}++$ and uses a complex system of physics condition lists, GEANT4 solid geometry, analyzer modules, and physics interaction libraries to perform primary event-generating Monte Carlo simulations. The software is also used to perform secondary level simulations, using the primary simulations as an input, with Fermilab specific FHiCl files.

The input files used for the simulations in this dissertation were generated by Yaqian Wang from stage 2 data recorded as hits on a virtual detector at the downstream end of the transport solenoid [73]. From these simulations the hit data was recorded at a virtual detector with a $2 \mathrm{~m}$ radius, positioned at $z_{m u 2 e}=38377.59 \mathrm{~mm}$ to cover the full solid angle from the field-of-view collimator, approximately $2 \mathrm{~m}$ upstream of the spotsize collimator (VD115) and the virtual detector positioned on the downstream edge of the spot-size collimator (VD101), the virtual detectors located at the STM detectors (VD89-90) are hidden within the shielding house.as shown in Figure 5.3. This data corresponds to $5.098 \times 10^{9}$ POT (Protons On Target), with a 131 pulse corresponding to a time of $222 \mu \mathrm{s}$. This corresponds to $3.89 \times 10^{7}$ POT over the $1695 \mathrm{~ns}$ extraction period. The kinetic energy spectrum for the hits associated with the relevant particles for this study, detected at VD115 can be seen in Figure 5.4. The plot for the photons has an expected number of high entries due to the high gamma-radiation environment, the plot for neutrons shows that there are only 336 neutron events detected across the entire $2.0 \mathrm{~m}$ radius virtual detector. Due to loss of solid-angle from the virtual detector to the spot-size collimator and geometric considerations from this input detector it can be determined that the statistics for neutrons will be low at the stopping-target monitor detectors. Figure 5.3 shows the virtual detector used for the input data set (VD115)

\subsubsection{Estimation of Neutron-Induced Radiation Damage}

Following from the work detailed within [64] an updated estimate for neutron-induced radiation damage is determined. Using the formula given for NIEL (5.5) originally 

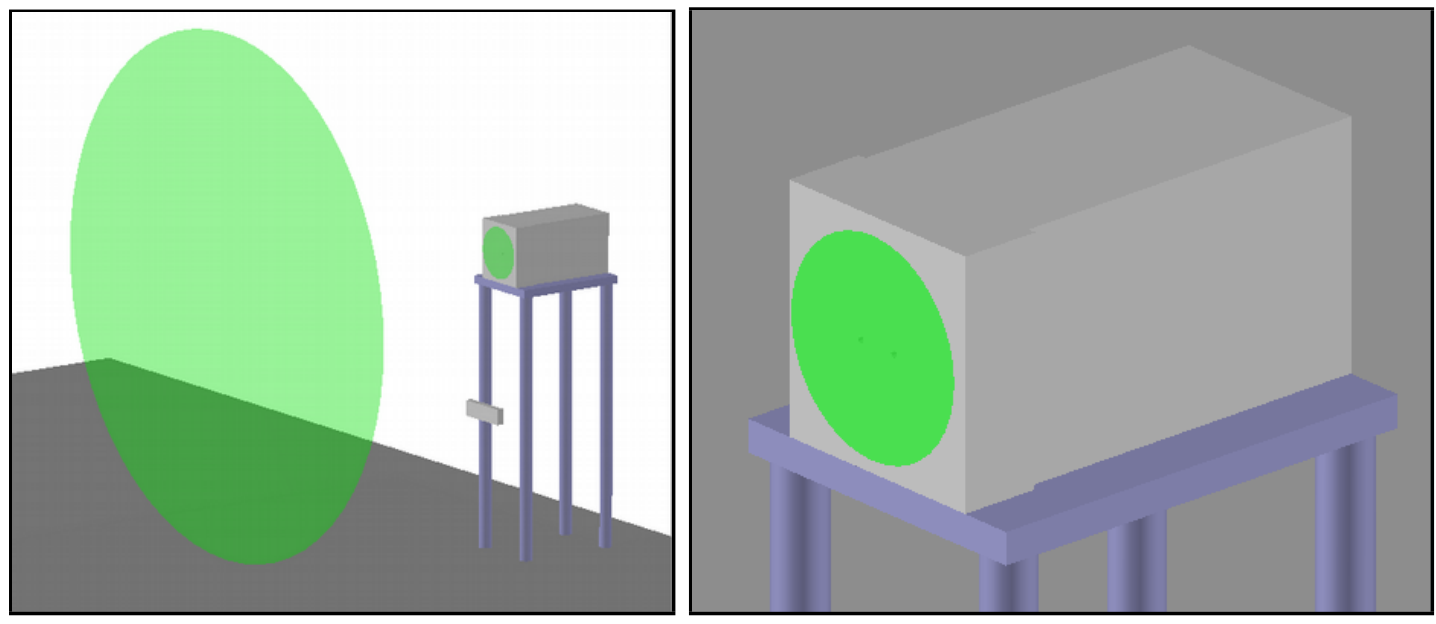

Figure 5.3: Geant4 geometry in Mu2e Offline with the Virtual Detectors VD115 and VD101 shown in green.
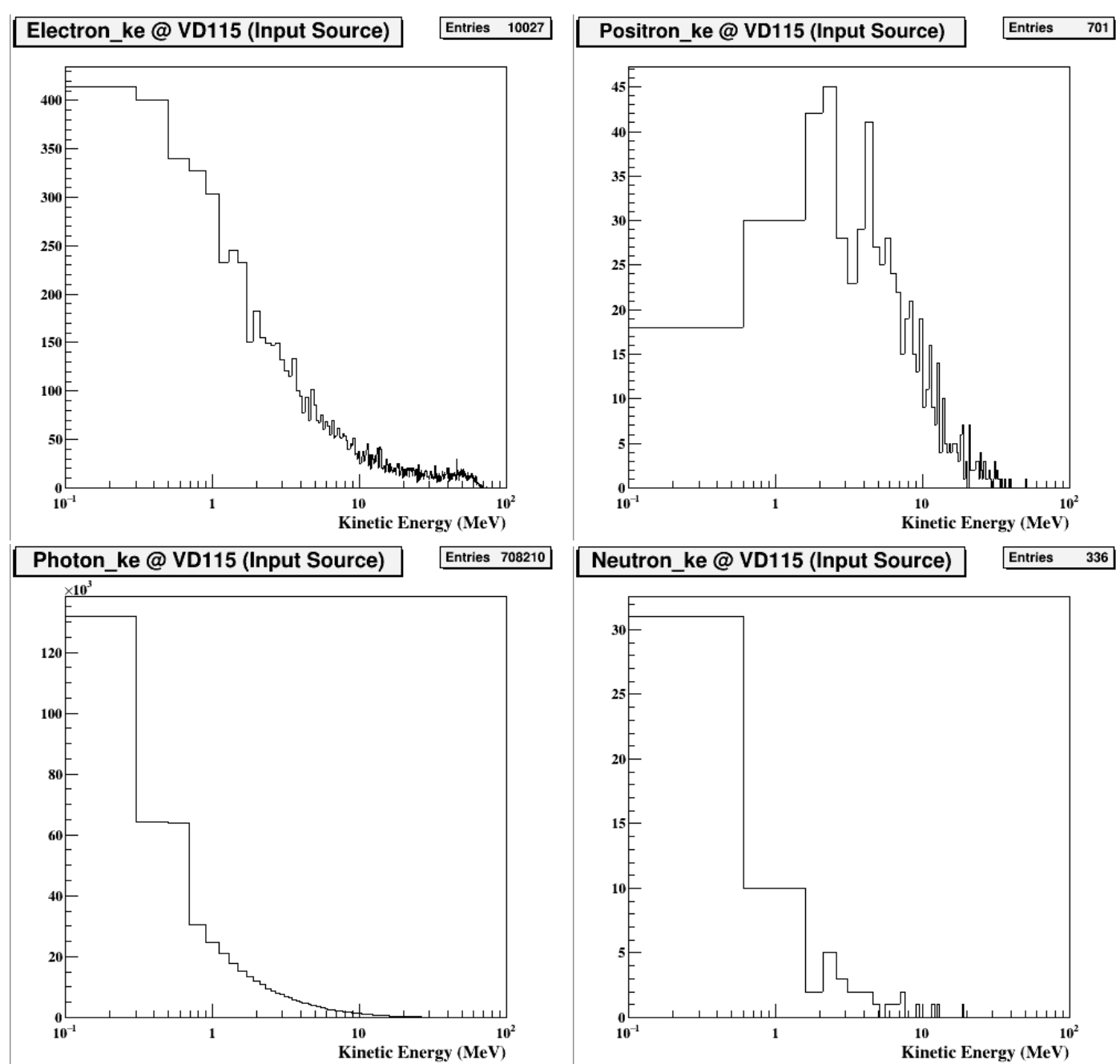

Neutron_ke @ VD115 (Input Source) Entries 336

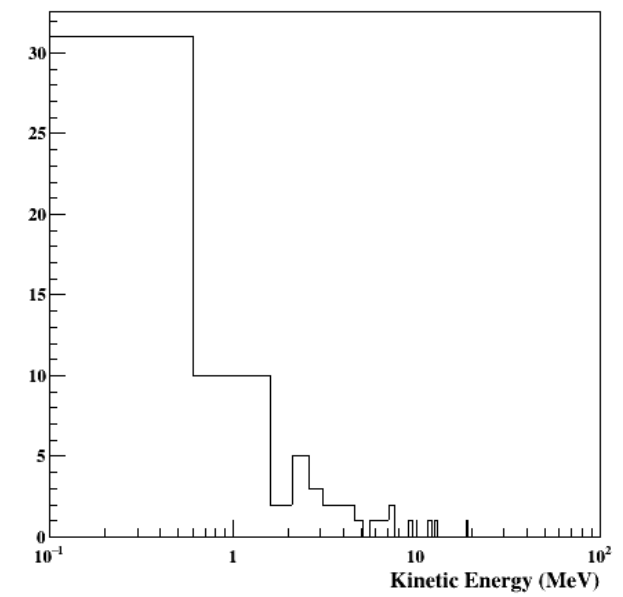

Figure 5.4: Kinetic energy spectrum for electrons and positrons (top row: left to right) and photons and neutrons (bottom row: left to right) detected at VD115 used as input source for simulations. 
intended for silicon and by extending it to germanium instead, a new quantity can be defined as the Kinetic-Energy Released in Matter (KERMA). KERMA takes into account the energy dependence of the incident flux and the cross-section information for the incident particle on the material which is critical for the estimation of radiation damage.

The expression for KERMA is given as [64]

$$
\operatorname{KERMA}_{\mathrm{n}}(\mathrm{keV}) \equiv K_{\mathrm{n}}=\operatorname{NIEL}_{\mathrm{n}}\left(\frac{\mathrm{keV} \cdot \mathrm{cm}^{2}}{\mathrm{~g}}\right) \times \phi\left(\frac{N_{\mathrm{n}}}{\mathrm{cm}^{2}}\right) \times m(\mathrm{~g})
$$

Where, $\phi$ is the incident particle flux given in $\frac{N_{\mathrm{n}}}{\mathrm{cm}^{2}}$ and $m(g)$ is the mass of the Germanium in grams. This can then be extended as a function of incident particle energy in the form

$$
\frac{\mathrm{d} K_{\mathrm{n}}\left(E_{\mathrm{n}}\right)}{\mathrm{d} E_{\mathrm{n}}}=\operatorname{NIEL}_{\mathrm{n}}\left(E_{\mathrm{n}}\right) \times \frac{\mathrm{d} \phi_{\mathrm{n}}\left(E_{\mathrm{n}}\right)}{\mathrm{d} E_{\mathrm{n}}} \times m
$$

Therefore to find the total energy released in the detector $\left(E_{R}\right)$, the integral of the KERMA function with respect to the incident particle energy is calculated as:

$$
E_{R}=\int \frac{\mathrm{d} K_{\mathrm{n}}\left(E_{\mathrm{n}}\right)}{\mathrm{d} E_{\mathrm{n}}} \mathrm{d} E_{\mathrm{n}}
$$

By measuring the energy resolution of a detector the KERMA function can then be determined. A study was carried out at the Lawrence Livermore Laboratory on a conventional p-type HPGe detector and a reverse bias (n-type) coaxial GMX HPGe detector fabricated from the same Ge crystal grown by ORTEC [74]. The crystals were placed $25.0 \mathrm{~cm}$ from a ${ }^{252} \mathrm{Cf}$ source, both, in their own separate $\mathrm{LN}_{2}$ cryostats maintained at approximately the same temperature. The ${ }^{252} \mathrm{Cf}$ source emitted $1.26 \times$ $10^{9}$ neutrons/s giving the detectors an approximate flux of $5.778 \times 10^{8} \mathrm{n} / \mathrm{cm}^{2} / \mathrm{s}$. The experimental procedure was to irradiate both detectors simultaneously with a known, measurable neutron flux and to measure the resolution of the $1332 \mathrm{keV} \gamma$-ray line for $\mathrm{a}^{60} \mathrm{Co}$ source. The detectors would then be irradiated again by the same neutron flux and, again, the resolution for the $\gamma$-ray line would be measured. This procedure was repeated until the detectors had a clear magnitude of difference in their sensitivities. // The results as shown in Figure 5.5 indicate the n-type GMX detector maintained a stable resolution of $\sim 1.8 \mathrm{keV}$ FWHM without damage to $3 \mathrm{keV}$ FWHM after being irradiated by $\mathcal{O}\left(10^{10}\right)$ neutrons. Due to the energy dependence of the radiation damage and the nature of the energy distribution of the neutrons generated at Mu2e 
being significantly to the energy distribution of the ${ }^{252} \mathrm{Cf}$ source, the same order of flux cannot be used for the Mu2e damage estimate, therefore it is necessary to calculate the KERMA function for the ORTEC study as this takes into account the cross sections for the respective incident particles.

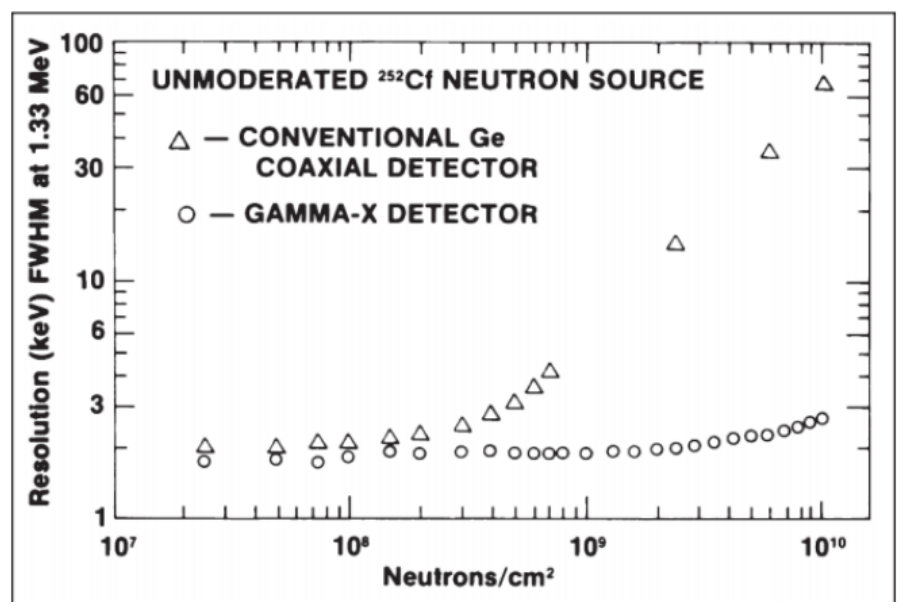

Figure 5.5: ORTEC study results of Resolution(FWHM) vs Neutron rate for a conventional p-type coaxial detector and a GMX n-type coaxial detector for neutrons from a ${ }^{252} \mathrm{Cf}$ source [56]. 
The bare neutron energy spectrum for neutrons from a ${ }^{252} \mathrm{Cf}$ source is then extracted (see Figures 5.6 and 5.7) and the NIEL for neutrons is extracted from the NIEL distributions for several incident particles (see Figure 5.8). This data was extracted using the plot digitization tool, WebPlotDigitizer [75]. Using the data from these two extractions and by taking a simple model of the mass of Germanium to be

$$
m_{G e}(g)=d_{G e}\left(\mathrm{~g} / \mathrm{cm}^{3}\right) \times \pi r^{2}\left(\mathrm{~cm}^{2}\right) \times h(\mathrm{~cm})=15.9 \mathrm{~g}
$$

where we have used; $d_{G e}=5.3 \mathrm{~g} / \mathrm{cm}^{3}, \pi r^{2}=1.0 \mathrm{~cm}^{2}$ and $h=3.0 \mathrm{~cm}$. Using the same procedure as 64, an expression for the neutron flux distribution can be obtained in the form

$$
\int \frac{\mathrm{d} \phi\left(E_{\mathrm{n}}\right)}{\mathrm{d} E_{\mathrm{n}}} \mathrm{d} E_{\mathrm{n}}=\frac{10^{10} \text { neutrons }}{\mathrm{cm}^{2}}
$$

We can then use the distributions shown in Figures 5.7 and 5.8 to normalise the KERMA as a function of neutron energy for the ORTEC study as shown in Figure 5.9. This takes the form

$$
E_{R}=\int \frac{\mathrm{d} K_{\mathrm{n}}\left(E_{\mathrm{n}}\right)}{\mathrm{d} E_{\mathrm{n}}} \mathrm{d} E_{\mathrm{n}}=\int \mathrm{NIEL}_{\mathrm{n}}\left(E_{\mathrm{n}}\right) \times \frac{\mathrm{d} \phi_{\mathrm{n}}\left(E_{\mathrm{n}}\right)}{\mathrm{d} E_{\mathrm{n}}} \times m=2.385 \times 10^{12} \mathrm{keV}
$$

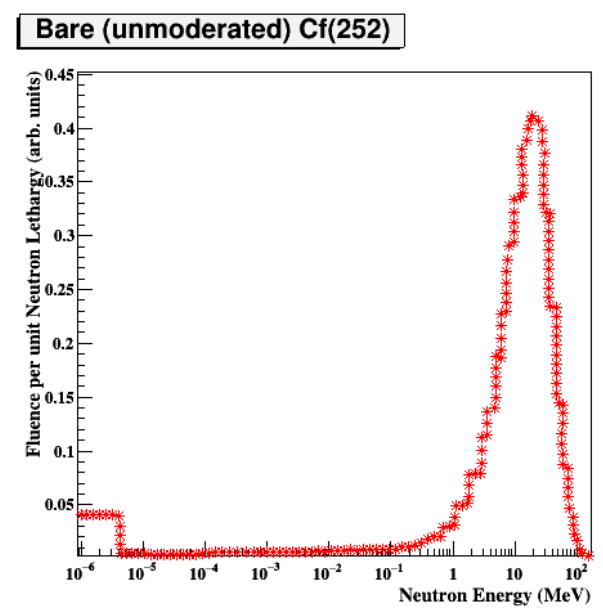

Figure 5.6: Neutron flux distribution from a bare ${ }^{252} \mathrm{Cf}$ source [64]. 

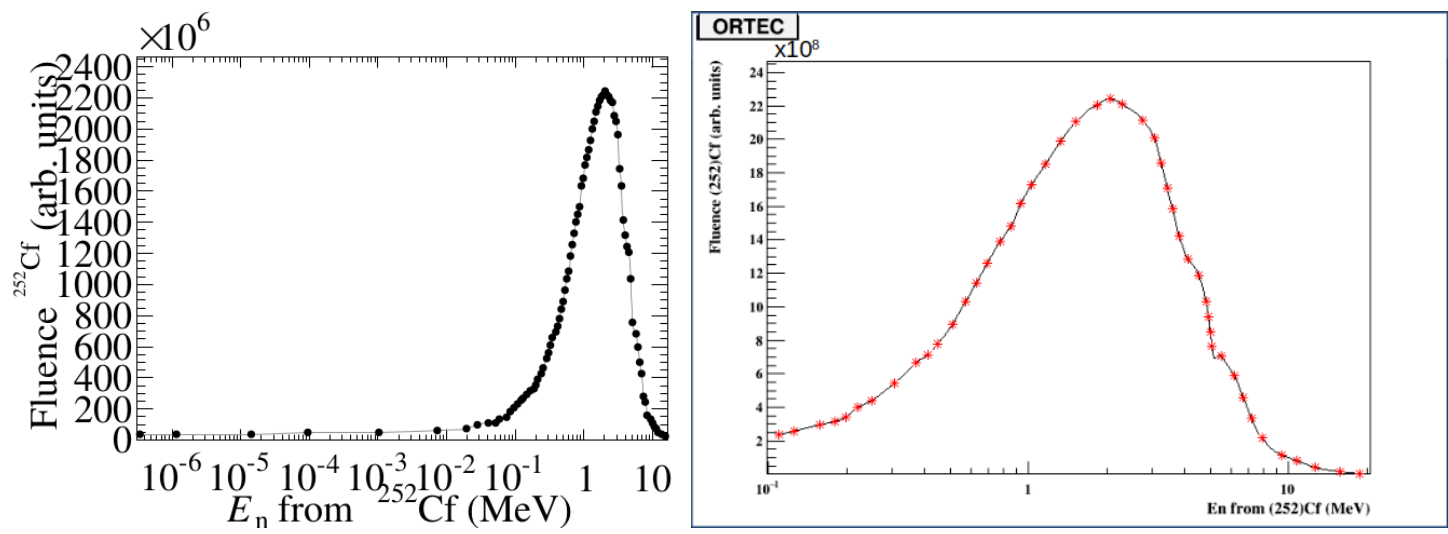

Figure 5.7: Extraction of Figure 5.6 obtained from [64 (left) and extraction used for calculations (right).
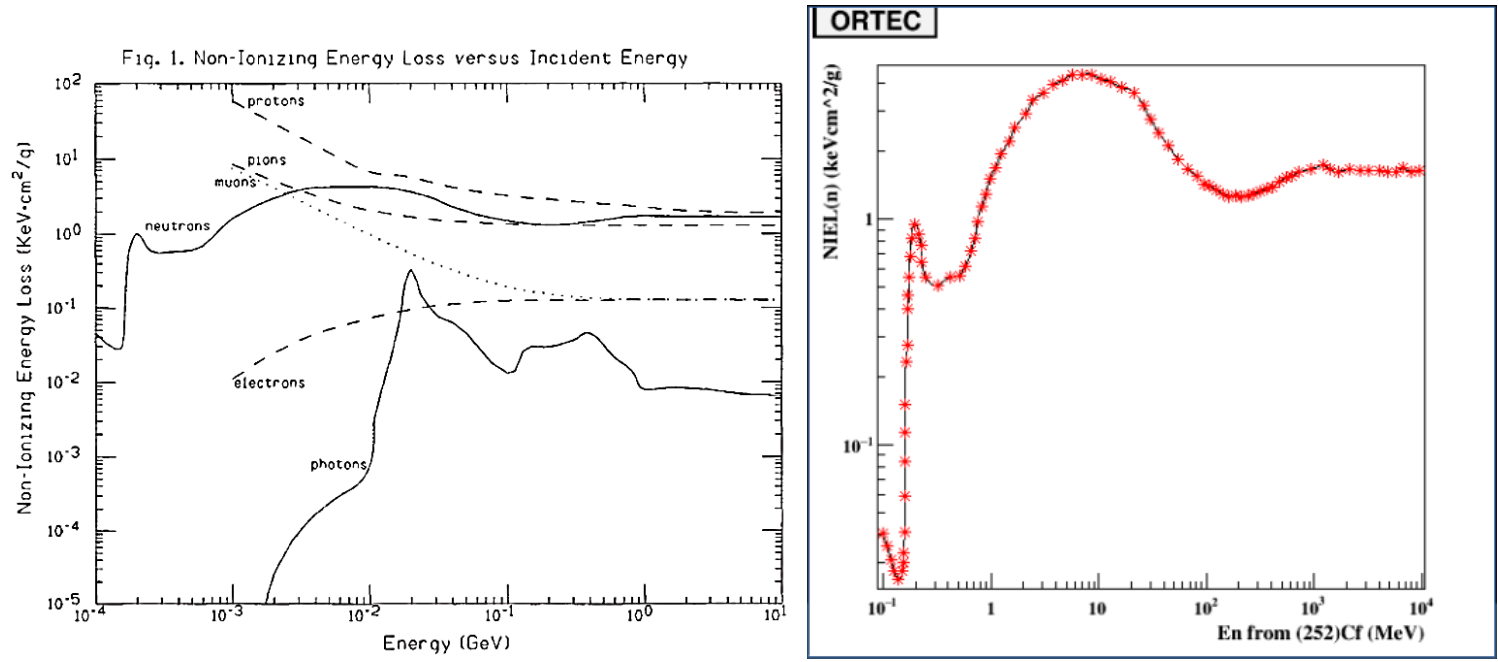

Figure 5.8: NIEL distribution for several particles as a function of incident energy ${ }^{252} \mathrm{Cf}$ source [64] (left) and extraction of neutron NIEL used for calculations (right).

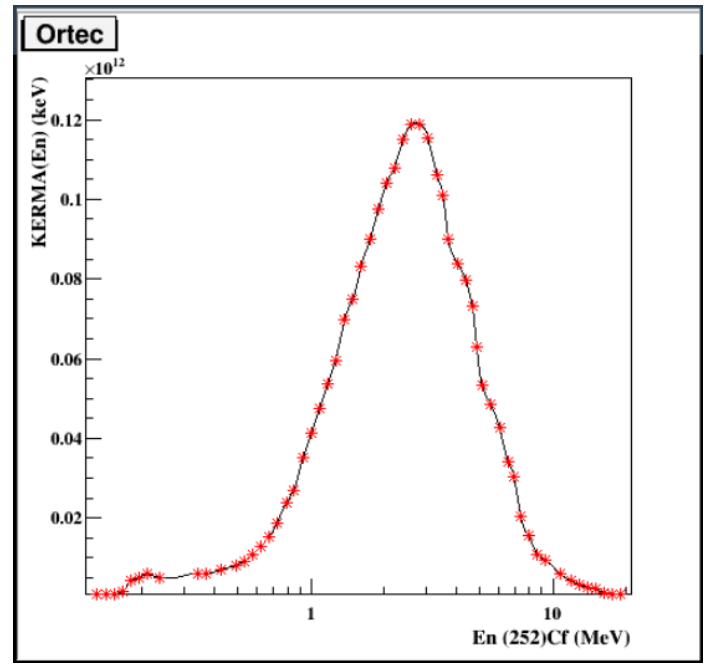

Figure 5.9: Calculated KERMA distribution as a function of energy for the ORTEC ${ }^{252} \mathrm{Cf}$ source radiation test. 
In the Mu2e beamline, the random processes responsible for the creation of the majority of fast neutrons happens within the Detector Solenoid with very few neutrons being created in the downstream area between the end-plug and the spot-size collimator. Because of this, it is therefore not possible to simply resample the neutrons exiting the detector solenoid as this would lead to the same events being resampled again and again with no new statistics generated. It is therefore more appropriate to take the data at a virtual detector that is directly on the upstream edge of the spot-size collimator (VD101). However, the statistics for fast neutrons measured at VD101 are extremely low in the simulation software due to geometric considerations and also due to the loss of solid angle [64] and it is therefore necessary to resample the events by applying a small Gaussian offset to the momentum in the z-direction $\left(p_{z}\right)$ on an event-by-event basis to get a smoother, more realistic approximation for the neutron distribution that would affect the detectors. 
The first resampling scheme that was used for estimating the KERMA function for Mu2e was the resampling scheme from [64] as follows

$$
\begin{array}{r}
\text { For }\left(1.0<p_{z}<175.0\right) \rightarrow p_{z(\text { new })}=p_{z}+\operatorname{rndm} \rightarrow \operatorname{Gaus}\left(0.0, p_{z} / 4.0\right) \\
\qquad \begin{array}{r}
\text { For }\left(p_{z}>175.0\right) \rightarrow p_{z(\text { new })}= \\
p_{z}+\operatorname{rndm} \rightarrow \operatorname{Gaus}(0.0,20) \\
\text { Otherwise } \rightarrow p_{z(\text { new })}=p_{z}
\end{array}
\end{array}
$$

The results for the resampled $z$-direction momentum distribution can be seen in Figure 5.10. By ensuring the Gaussian offset was only applied when the momentum was above $1 \mathrm{MeV}$ the resampling scheme only factored in forward going neutrons as well as ensuring only the fast neutrons were resampled and not the low energy thermal neutrons. Only the momentum in the $z$-direction was resampled due to the momentum in the $x$ and $y$ direction being highly peaked around $1 \mathrm{MeV}$ and the change in the Gaussian offset from $p_{z} / 4$ to 20 when $p_{z}>175.0$ is due to the decreased spread of data in this region. The resampling scheme was repeated for two sets of primary event data sets generated using separate input files to ensure that the results were as close to being invariant as possible for two separate seeds. From Figure 5.10 it can be seen that the resampled scheme follows the same distribution for the backwards going and thermal neutrons $(<1 \mathrm{MeV})$. Beyond the $1 \mathrm{MeV}$ point where the resampling scheme begins to apply the offset the momentum can be seen to follow a smoothed distribution of the primary event momentum data with a small peak between 0 and $50 \mathrm{MeV}$ due to the density of the data in this region as a feature of the Gaussian smearing. Using the newly generated, resampled, momentum data the kinetic energy could then be calculated as follows

$$
E_{k}=\sqrt{\left(\left(p_{x}^{2}+p_{y}^{2}+p_{z(n e w)}^{2}\right)+m_{n}^{2}\right)}-m_{n}
$$

Where $p_{x}$ and $p_{y}$ are the original non-resampled momentum data in the respective planes, $p_{z(\text { new })}^{2}$ is the newly resampled neutron momentum data and, $m_{n}$ is the rest mass of the neutron. From this kinetic energy function the resampling and smoothing could then be set to the form

$$
\int \frac{\mathrm{d} K_{\mathrm{n}}\left(E_{\mathrm{n}}\right)}{\mathrm{d} E_{\mathrm{n}}} \mathrm{d} E_{\mathrm{n}}(\mathrm{Mu} 2 \mathrm{e})=\int \frac{\mathrm{d} K_{\mathrm{n}}\left(E_{\mathrm{n}}\right)}{\mathrm{d} E_{\mathrm{n}}} \mathrm{d} E_{\mathrm{n}}\left(\text { ORTEC }{ }^{252} \mathrm{Cf}\right)
$$



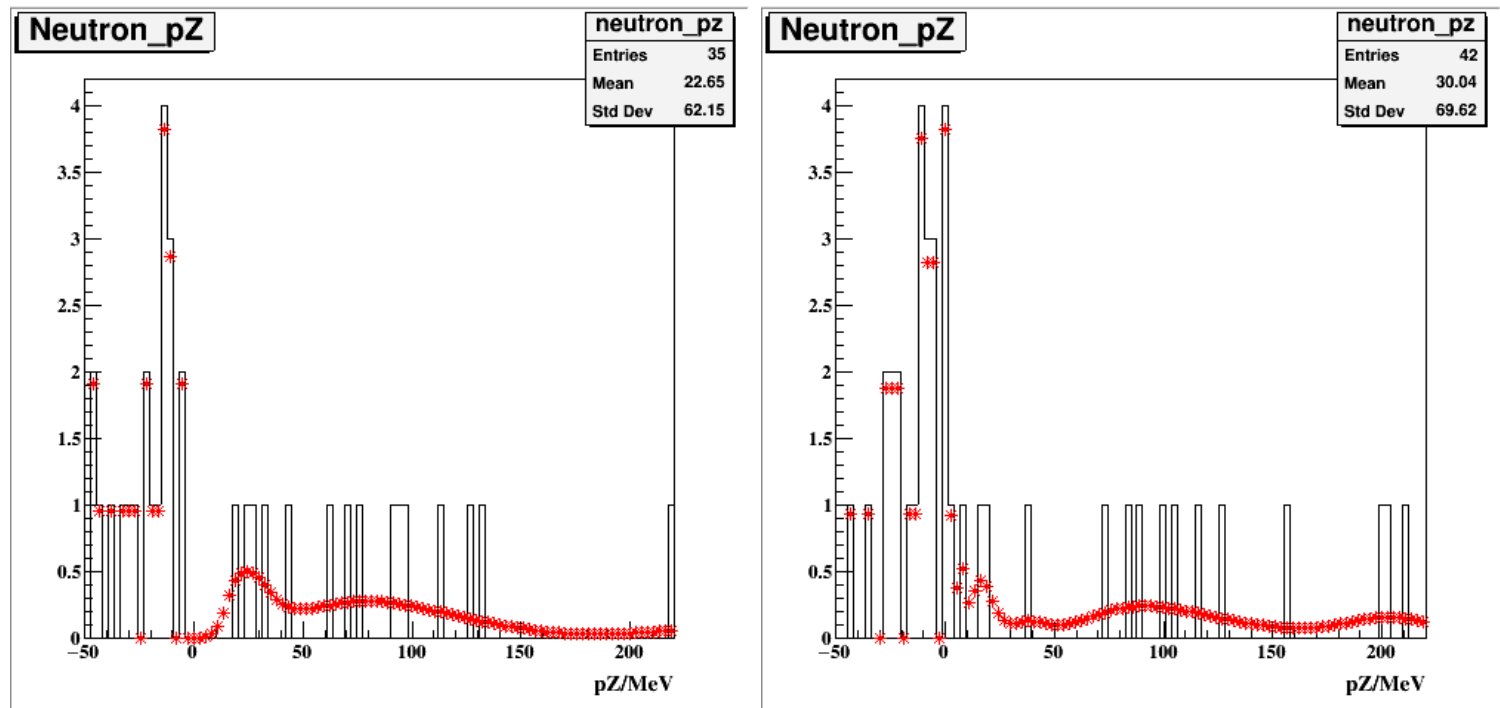

Figure 5.10: Momentum distribution of primary neutron data with the resampled data plotted in red for 35 events and 42 events respectively, demonstrating the smoothing of the resampling scheme.

Using this normalisation function the KERMA can then be calculated from

$$
\begin{array}{r}
\int \frac{\mathrm{d} K_{\mathrm{n}}\left(E_{\mathrm{n}}\right)}{\mathrm{d} E_{\mathrm{n}}} \mathrm{d} E_{\mathrm{n}}(\mathrm{Mu} 2 \mathrm{e})=\int \operatorname{NIEL}_{\mathrm{n}}\left(E_{\mathrm{n}}\right) \times \frac{\mathrm{d} N_{\mathrm{n}}^{\mathrm{Mu} 2 \mathrm{e}}\left(E_{\mathrm{n}}\right)}{\mathrm{d} E_{\mathrm{n}}}\left(\mathrm{cm}^{-2}\right) \times m(g) \\
=2.385 \times 10^{12} \mathrm{keV}
\end{array}
$$

From this expression, using the newly calculated KERMA function, the normalization of the distribution for the number of neutrons $\mathrm{d} N_{\mathrm{n}}^{\mathrm{Mu} 2 \mathrm{e}}\left(E_{\mathrm{n}}\right)$ can be found. Due to the extremely low statistics generated in the simulations and the nature of the resampling scheme, it becomes difficult to resample up to the number of neutrons that would be required to produce the amount of KERMA measured; therefore, the scaling behaviour of the KERMA with respect to the number of neutrons generated has been adopted to approximate the normalization for the number of neutrons. The primary data was resampled to create $10^{6}$ and $10^{7}$ neutrons, from this the scaling behaviour was extracted and then the KERMA for the $\mathcal{O}\left(10^{6}\right)$ resampled data was scaled up to the KERMA obtained from the ORTEC extraction. From this the normalization for the number of neutrons that would be required to impart this level of KERMA could be solved for. Using this method it was found that for 35 primary neutrons resampled the rate of neutrons required to impart the same level of KERMA as the ORTEC extraction was $6.8 \times 10^{10}$ neutrons and for 42 primary neutrons a rate of $7.5 \times 10^{10}$ neutrons. Here it can be seen that there is not perfect invariance in the results; however, this process of resampling has high levels of uncertainty and therefore this level of difference is 
to be expected. Finding the average of these two values to be $7.2 \times 10^{10}$ neutrons and by taking the neutron flux rate on the detectors for $5 \times$ beam flash statistics as given in [64] to be 354 neutrons $/ \mathrm{cm}^{2} / \mathrm{s}$. It is found that the time before annealing is absolutely necessary is approximately 78 months of run time assuming an average month is 30 days, this is $\sim 4.6$ times greater than the previously calculated 17 months indicating that there is significant improvements to the reduction of radiation damage with the updated geometry and simulation process since the 2016 study [64].
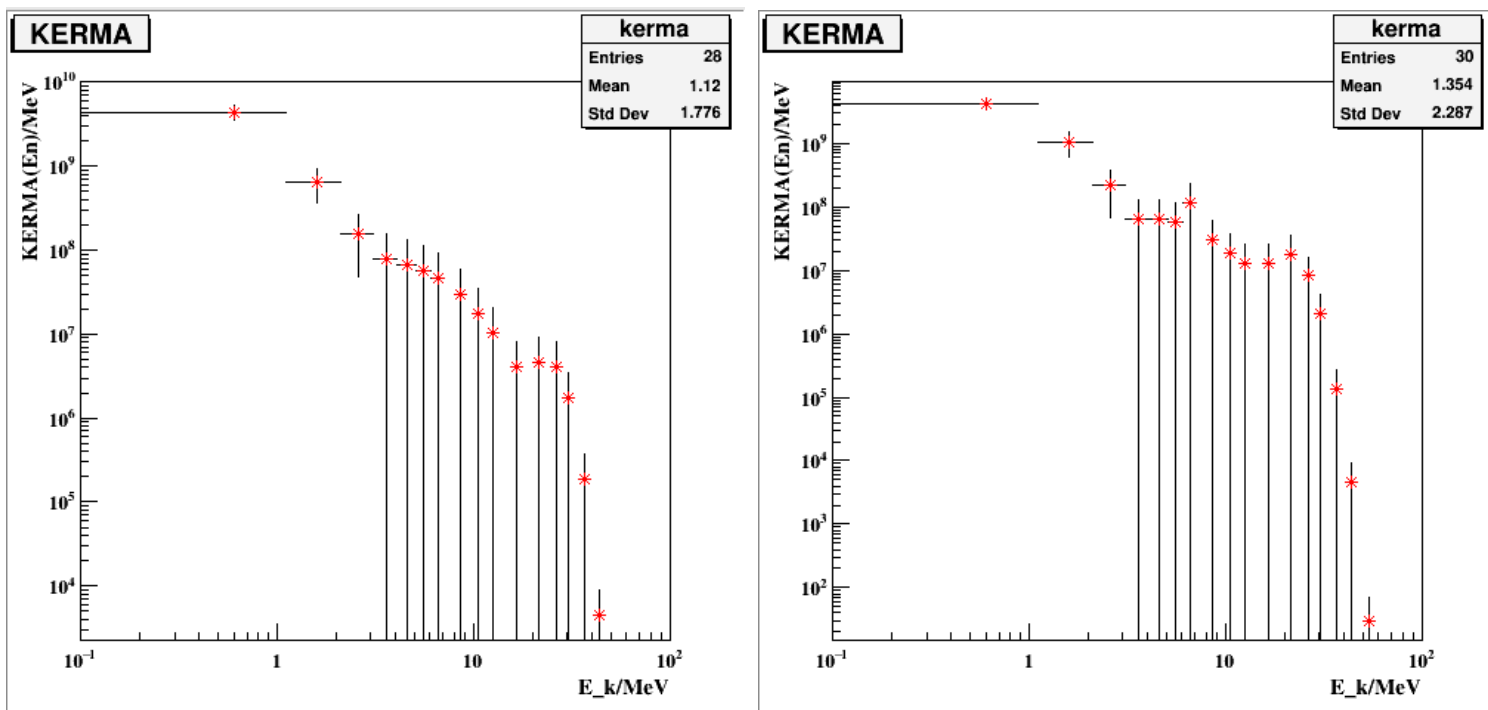

Figure 5.11: KERMA as a function of neutron energy from 35 and 42 events resampled respectively using the 2016 resampling scheme'.

Due to the nature of the simulations being a staged process with input data, it was necessary to ensure that neutrons with negative momenta that had reached VD101 (virtual detector placed directly on the upstream edge of the Spot-Size Collimator) did not have any significant impact on the results due to the possibility of them being forward-going in an earlier stage. To ensure this as well as an attempt to fit the resampling scheme more accurately to the input data, a new four-Gaussian resampling scheme was tested by selecting three cuts on the dataset: the first selects events with high backwards going momentum around the mean of the dataset $<-30 \mathrm{MeV}$ to capture very fast neutrons that may have been forwards-going in an earlier stage, the second cut is the central cut which determined the first cut region and allows the capture of neutron events with fast backwards going momenta centred around the mean $<1 \mathrm{MeV}$ and finally the third cut, like the previous resampling scheme selects 
the fast forwards going neutrons. The resampling scheme is as follows.

$$
\begin{array}{r}
\text { For }\left(p_{z}<-30.0\right) \rightarrow p_{z(\text { new })}=\operatorname{rndm} \rightarrow \operatorname{Gaus}\left(\mu_{1}, \sigma_{1}\right) \\
\text { For }\left(-30.0<p_{z}<1.0\right) \rightarrow p_{z(\text { new })}=\operatorname{rndm} \rightarrow \operatorname{Gaus}\left(\mu_{2}, \sigma_{2}\right) \\
\text { For }\left(1.0<p_{z}<175\right) \rightarrow p_{z(\text { new })}=\operatorname{rndm} \rightarrow \operatorname{Gaus}\left(\mu_{3}, \sigma_{3}\right) \\
\text { Otherwise } \rightarrow p_{z(\text { new })}=p_{z}+\operatorname{rndm} \rightarrow \operatorname{Gaus}(0.0,20.0)
\end{array}
$$

where $\left(\mu_{i}, \sigma_{j}\right)$ denotes the mean and standard deviation of $p_{z}$ for the data sets calculated within the respective cut regions, with $p_{z}$ given in $\mathrm{MeV}$.

\begin{tabular}{|l|l|l|l||l|l|l|}
\hline \multicolumn{5}{|c|}{35 events resampled } & \multicolumn{4}{|c|}{42 events resampled } \\
\cline { 1 - 3 } $\mathrm{i}$ & $\mu_{i}$ & $\sigma_{i}$ & $\mathrm{i}$ & $\mu_{i}$ & $\sigma_{i}$ \\
\hline$i=1$ & -41.0 & 6.29 \\
\hline$i=1$ & -44.6 & 7.50 \\
\hline$i=2$ & -16.4 & 7.77 \\
\hline$i=3$ & 72.3 & 38.8 \\
\end{tabular}$\quad$\begin{tabular}{lllll}
$i=2$ & -12.1 & 8.97 \\
\hline
\end{tabular}

Table 5.1: Values of parameters calculated for the Gaussian functions used in fourGaussian resampling scheme

Once again the input data for 35 and 42 primary neutron events was resampled with the parameters for the Gaussian functions as shown in Table 5.1. The same scaling behaviour as previously outlined was used to scale up to the normalised neutron rate required to impart the same level of KERMA as the ORTEC study. This can be seen in 5.12 . Using this resampling scheme the number of neutrons was found to be $6.5 \times 10^{10}$ neutrons and $7.3 \times 10^{10}$ neutrons for the 35 and 42 primary events respectively. Taking the average to be $6.9 \times 10^{10}$ neutrons and using the same flux rate of 354 neutrons $/ \mathrm{cm}^{2} / \mathrm{s}$ the time before annealing would be required is calculated to be approximately 75 months assuming an average month to be 30 days. This gives between the two resampling schemes an estimated time of 75 and 78 months. Due to the high level of uncertainty in both the resampling process and the extraction of the ORTEC data, as well as geometric considerations and the staged nature of the simulations, this time is only an estimate and annealing should be carried out on a much more regular basis to ensure full, optimal detector performance is maintained during the experiment run-time. 

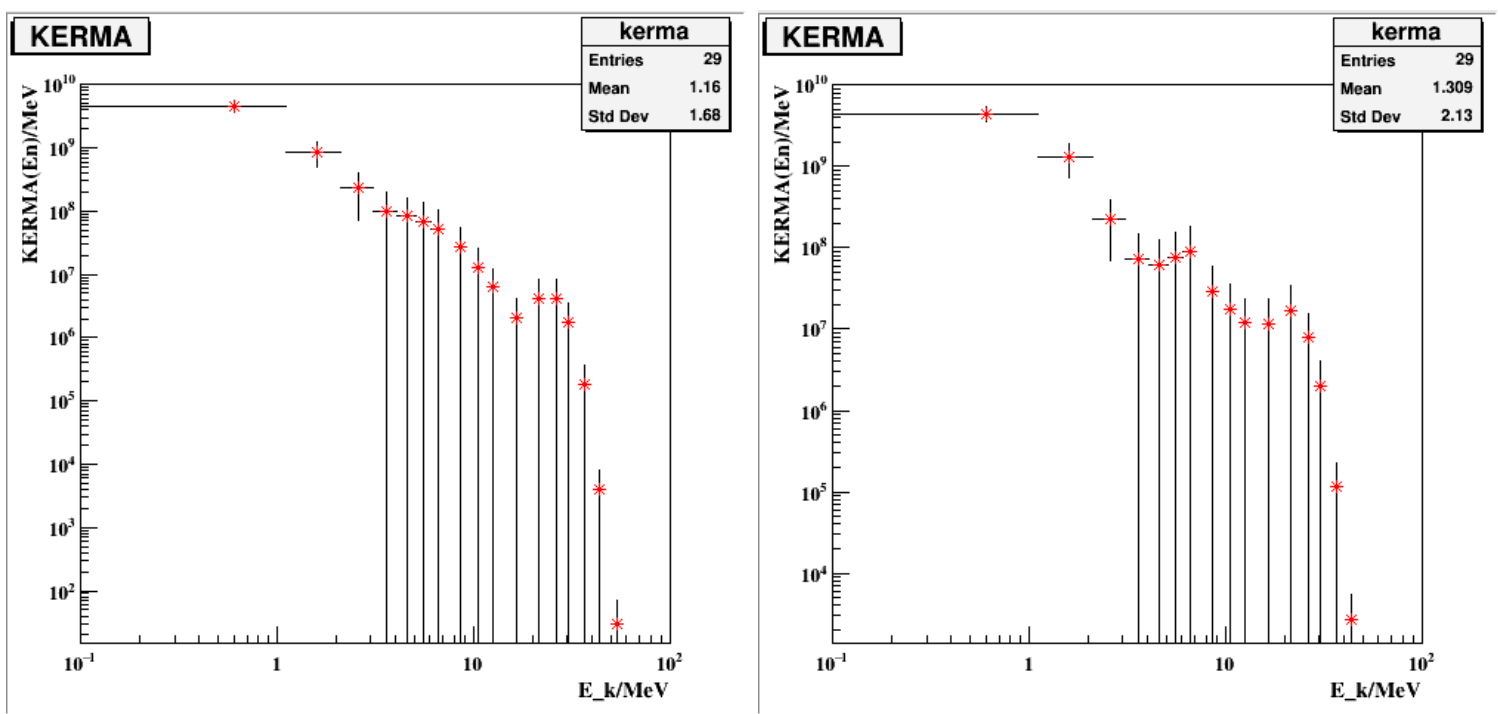

Figure 5.12: KERMA as a function of neutron energy from 35 and 42 events resampled respectively using four-Gaussian resampling scheme'.

\subsubsection{Shielding Effects of Spot-Size Collimator for Fast Neutrons}

As the statistics for the neutron flux in the simulations is so low it is not possible to determine the rate at which neutrons pass through the spot-size collimator and reach the detectors; therefore, a Geant4 neutron particle gun is placed at in the geometry at coordinates - Pos $_{\mathrm{mu} 2 \mathrm{e}}=(-3904 \mathrm{~mm}, 0 \mathrm{~mm}, 38350 \mathrm{~mm})$, corresponding to the centre of the stopping target and approximately $2 \mathrm{~m}$ upstream of the Spot-Size Collimator. The momentum is set at $43.360 \mathrm{MeV}$ which corresponds to $E_{k}=1 \mathrm{MeV}$ in accordance with ASTM E722-14. [71] A $5 \mathrm{keV}$ upper and lower limit is set and the momentum is distributed with a Gaussian distribution, using the FWHM rule to find the $\sigma$-parameter for the Gaussian function, with a full-width of $2 \mathrm{keV}$.

We first take the general equation for a Gaussian function in the form

$$
f(x)=C e^{\left(-x^{2} / 2 \sigma^{2}\right)}
$$

If we then let $x=h$ at half the maximum height of the Gaussian peak then

$$
0.5 C=C e^{\left(-x^{2} / 2 \sigma^{2}\right)} .
$$

By taking the natural logarithm of both sides we arrive at the relationship:

$$
h^{2}=-2 \ln (0.5) \sigma^{2}=2 \ln 2 \sigma^{2} .
$$


as $x=h$ at half the maximum height the full width $\Gamma$ is equal to $2 h$ and thus:

$$
\Gamma=2 \sqrt{2 \ln 2} \sigma=2.355 \sigma \rightarrow \sigma=2 \mathrm{keV} / 2.355
$$

To test the rates two sets of data were generated the first with $\mathcal{O}\left(10^{7}\right)$ and the second with $\mathcal{O}\left(10^{8}\right)$ neutrons generated to ensure invariance except for a scaling factor. The time parameters for the neutron generation were set to $t=0.0$ and the rate of neutron flux at VD101 and VD89|90 was recorded. A cut on momentum of $\left(p_{z}>0.0\right)$ was made to separate forward going neutrons from those that recoil from the collimator surface the results from this can be seen in Figure 5.13 .
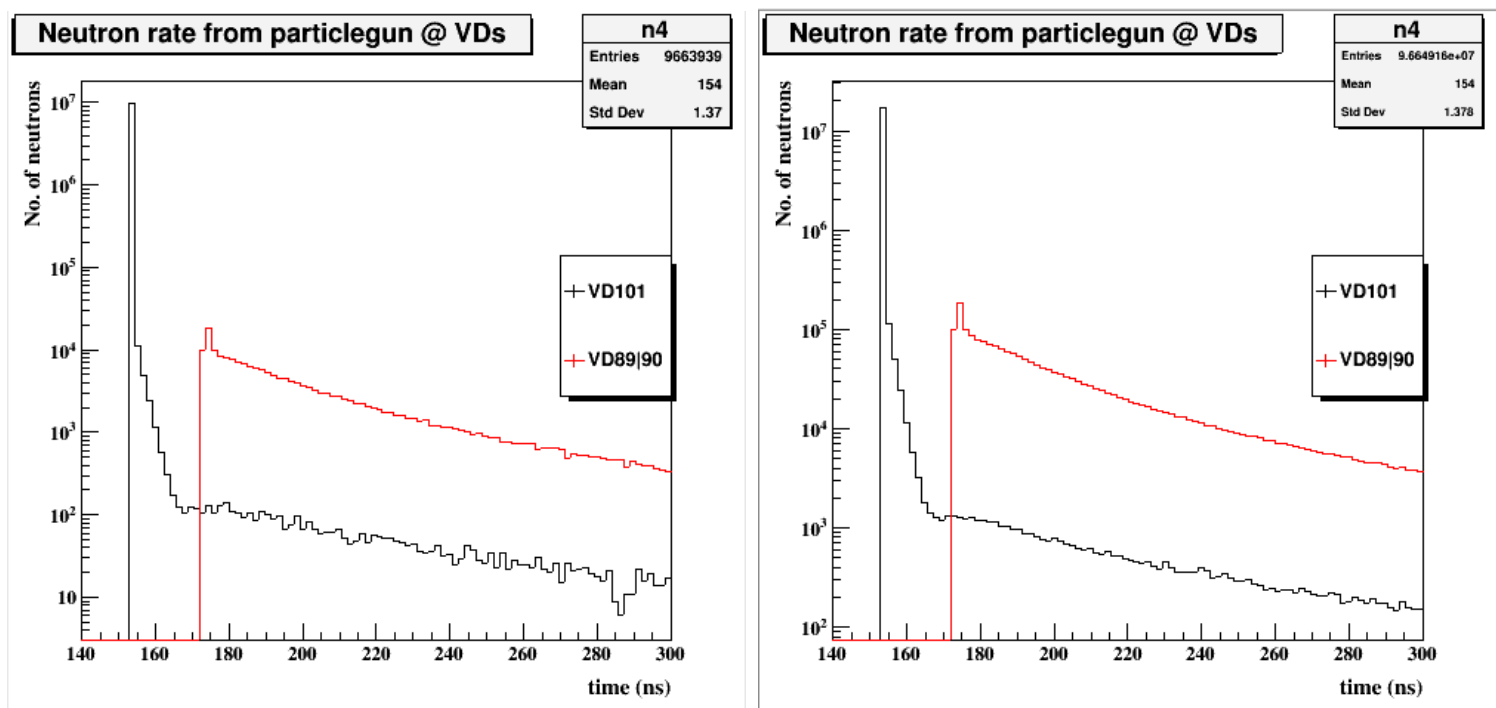

Figure 5.13: $1 \mathrm{MeV}$ neutron flux rate at virtual detectors from G4 particleGun for $\mathcal{O}\left(10^{7}\right)$ and $\mathcal{O}\left(10^{8}\right)$ events respectively.

It can be seen from these results that the results are indeed only affected by a scaling factor and thus it is no longer necessary to use the $\mathcal{O}\left(10^{8}\right)$ data. The fast neutrons reach the pot-Size collimator approximately 150ns after generation, the rate then rapidly drops off by $4-5$ orders of magnitude and continues to decline as time increases. The likely source of the neutrons after the initial peak is primarily from elastic scattering effects with atoms in the air with contributions also from inelastic scattering effects. The neutrons then reach the Virtual Detectors located at the STM detector positions approximately $10 \mathrm{~ns}$ later with an initial magnitude of 2-3 orders lower than at VD101 with a slow decline in the rate as time increases due to the effects of interaction within the collimator apertures reducing the energy of the neutrons.

To test this the kinetic energy of the forward-going neutrons was also measured as 
shown in Figure 5.14 .
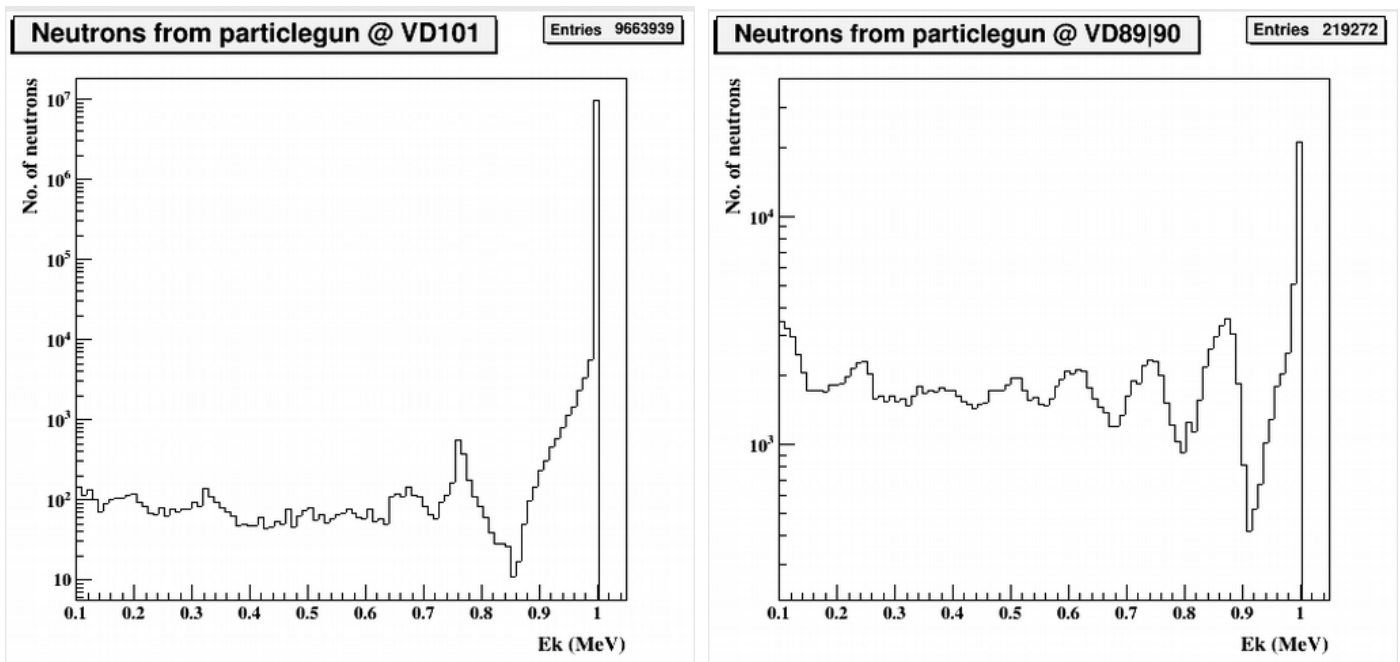

Figure 5.14: $1 \mathrm{MeV}$ neutron kinetic energy spectrum at virtual detectors from G4 particleGun for $10^{7}$ events at VD101 and VD89-90 (Left and right respectively).

The kinetic energy spectra for VD101 show that the majority of neutrons maintain the initial $1 \mathrm{MeV}$ energy and the rate for the slowed neutrons due to the scattering effects is fairly consistent at four orders of magnitude for all energies below $1 \mathrm{MeV}$. We can see that the kinetic energy spectra for virtual detectors VD89 - 90 still have the largest peak at $1 \mathrm{MeV}$; however, the rates for the slowed neutrons is much higher with less than an order of magnitude difference, due to the slowing effects of both the scattering processes with atoms in the air as well as interactions with the collimator as they pass through the apertures. Up to $1 \mathrm{MeV}$ the elastic scattering cross sections for light elements, such as those in air, are more-or-less independent of the neutron energy. These results show that $2.27 \%$ of the $1 \mathrm{MeV}$ neutrons passed through the Spot-Size collimator; therefore these neutrons are not the primary cause of significant damage to the detectors.

The kinetic energy distribution from the data used with the resampling schemes has neutrons with kinetic energy up to a maximal value between $50 \mathrm{MeV}$ and $60 \mathrm{MeV}$. These very fast neutrons behave differently to $1 \mathrm{MeV}$ neutrons, therefore it was necessary to test the effects of the shielding from the collimator within this extrema. The momentum for the particle gun therefore was set to $326.155 \mathrm{MeV}$ which corresponds to a kinetic energy of $55 \mathrm{MeV}$. This was then distributed again with a FWHM Gaussian function however this time a $2 \mathrm{MeV}$ full-width was used, to increase the spread, corresponding to $\sigma=2 \mathrm{MeV} / 2.355$. A $5 \mathrm{MeV}$ limit was set on the kinetic energy to 
ensure the distribution was spread between $50 \mathrm{MeV}$ and $60 \mathrm{MeV}$. With a minimum limit on the momentum of $310.574 \mathrm{MeV}$ and a maximum limit of $341.098 \mathrm{MeV}$. The rates for these very fast neutrons can be seen in Figure 5.15 .

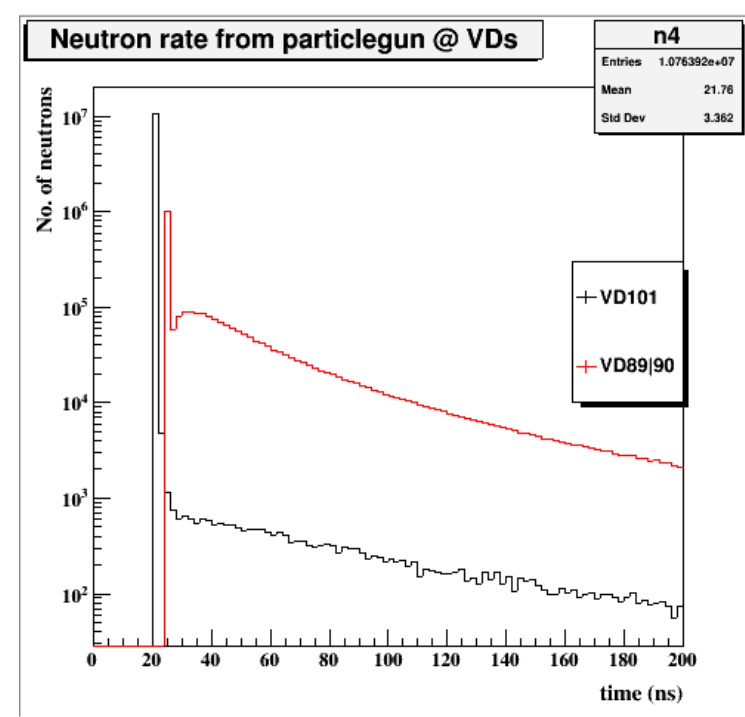

Figure 5.15: High energy neutron flux rate at virtual detectors from G4 particleGun for $\mathcal{O}\left(10^{7}\right)$ events.

These neutrons reach VD101 in approximately 20 ns and traverse the collimator aperture in approximately 2.5 ns with $27.12 \%$ of the neutrons passing successfully reaching VD89 - 90. The kinetic energy spectra for these neutrons can be seen in Figure 5.16 .
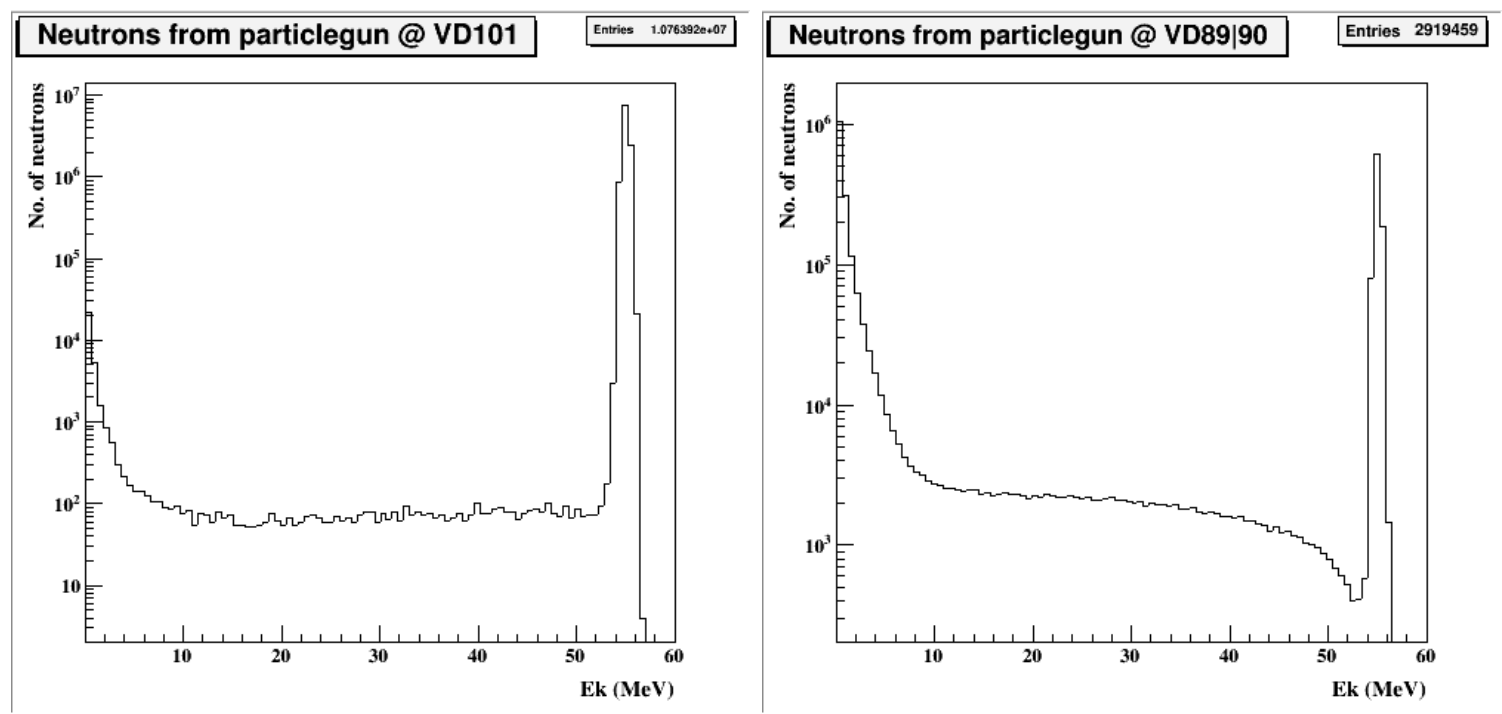

Figure 5.16: High energy neutron kinetic energy spectrum at VD101 and VD89-90 (left and right respectively) from G4 particleGun for $\mathcal{O}\left(10^{7}\right)$ events.

These spectra show that the rate is maximal at $55 \mathrm{MeV}$ with an even spread either 
side, the rate then drops off significantly due to small energy losses and stays at a consistent rate down to $<10 \mathrm{MeV}$ where the rate increases again. This is due to the dominant process for these high energy neutrons being inelastic scattering where the slowing process rate is significantly increased due to the loss of energy from the emission of a neutron from the excited atomic nucleus as well as the emission of a photon. The slowing effects are even more significant after traversing the Spot-Size apertures as the neutrons are more likely to interact with the tungsten atoms in the collimator wall.

The dominating inelastic scattering can be seen in the order of difference in the kinetic energy spectra of the photons produced by the neutrons, where an $\mathcal{O}(10)$ and $\mathcal{O}(30)$ increase in the number of photons produced can be seen from Figure 5.17. These very fast neutrons are the most likely to cause significant radiation damage therefore it is important to stress that the time calculated from the resampling data is a conservative estimate and annealing should take place much more regularly than the estimated time with respect to the ORTEC data.

\subsubsection{Estimation of Positron-Induced Radiation Damage}

As well as the radiation damage caused by neutrons it is also important to highlight the fact that there is a high energy gamma background, due to the high energies pair production can occur within close proximity to the STM detectors and therefore it is also important to make an estimate on the time before annealing would be required due to these processes. As the NIEL takes into account cross-sections for various particle species it is possible to extract the NIEL from Figure 5.8 for electrons and extend the KERMA formula from the ORTEC study to the case of positrons. This extraction can be seen in Figure 5.18a. We therefore get the expression

$$
\int \frac{\mathrm{d} K_{\mathrm{e}+}\left(E_{\mathrm{n}}\right)}{\mathrm{d} E_{\mathrm{n}}} \mathrm{d} E_{\mathrm{e}+}(\mathrm{Mu} 2 \mathrm{e})=\int \frac{\mathrm{d} K_{\mathrm{n}}\left(E_{\mathrm{n}}\right)}{\mathrm{d} E_{\mathrm{n}}} \mathrm{d} E_{\mathrm{n}}\left(\text { ORTEC }{ }^{252} \mathrm{Cf}\right)
$$

It is important to note that the rate of photons was calculated in [64], which should take into account the gammas that pair-produce and the NIEL from those electrons and positrons. This study provided an estimate of $\sim 9.1 \pm 0.9$ years before annealing would be required. However due to the large number of positrons formed in updated simulations it was important to test that there had not been any significant decrease 

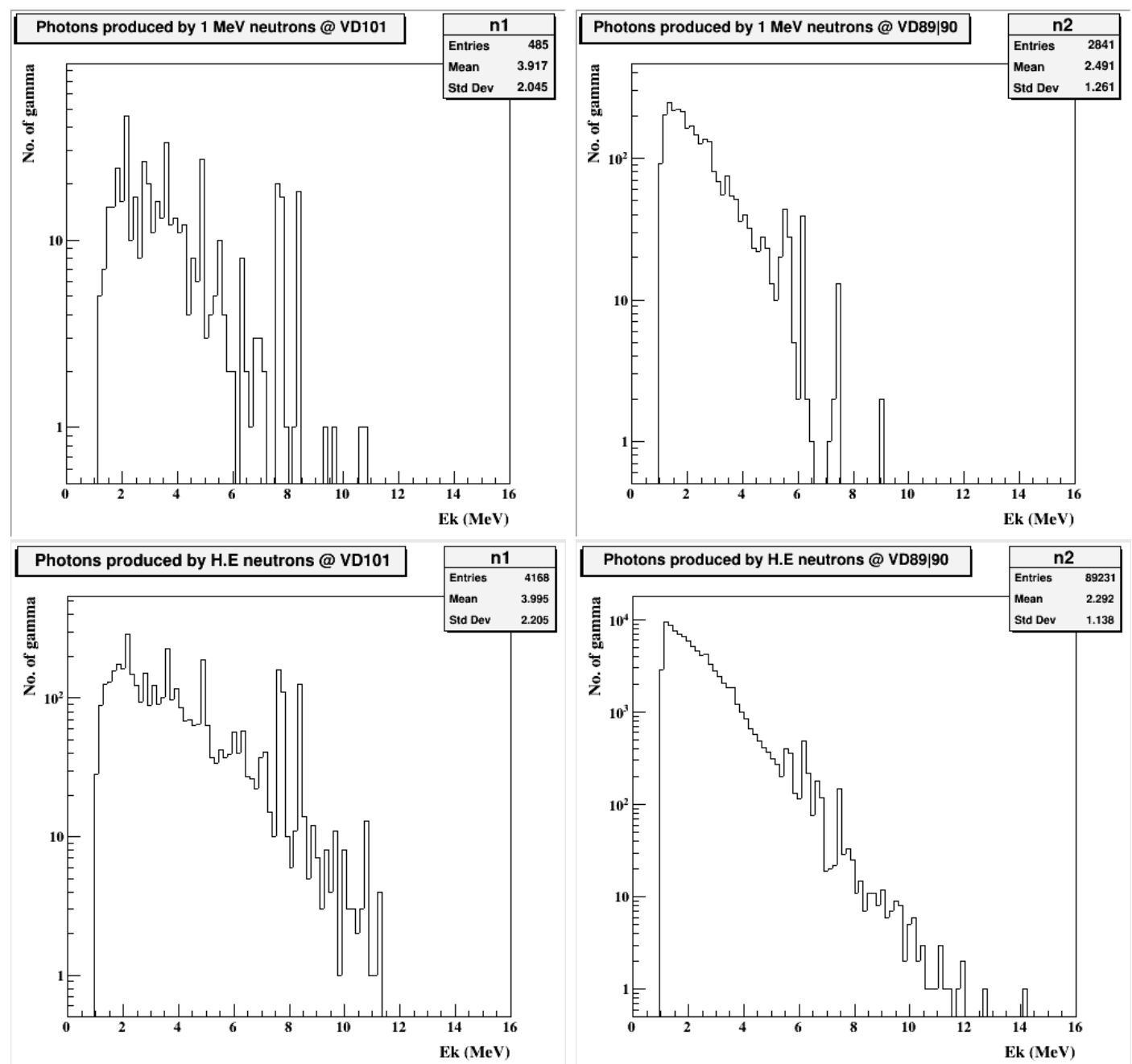

Figure 5.17: Kinetic energy spectrum of photons at virtual detectors VD101 and VD89 (left and right respectively) produced by $1 \mathrm{MeV}$ and high-energy neutrons (top and bottom row respectively) from $\mathrm{G} 4$ particleGun for $\mathcal{O}\left(10^{7}\right)$ events.

in this time. The 23,936 primary positrons from the simulation are distributed, using the same Gaussian offset as the first resampling scheme in the previous section, in the form

$$
\begin{array}{r}
\int \frac{\mathrm{d} K_{\mathrm{e}+}\left(E_{\mathrm{n}}\right)}{\mathrm{d} E_{\mathrm{n}}} \mathrm{d} E_{\mathrm{e}+}(\mathrm{Mu} 2 \mathrm{e})=\int \mathrm{NIEL}_{\mathrm{e}+}\left(E_{\mathrm{e}+}\right) \times \frac{\mathrm{d} N_{\mathrm{e}+}^{\mathrm{Mu} 2 \mathrm{e}}\left(E_{\mathrm{e}+}\right)}{\mathrm{d} E_{\mathrm{e}+}}\left(\mathrm{cm}^{-2}\right) \times m(g) \\
=2.38537 \times 10^{12} \mathrm{keV}
\end{array}
$$

Once again by solving for the normalization $\left(\mathrm{d} N_{\mathrm{e}+}^{\mathrm{Mu} 2 \mathrm{e}}\left(E_{\mathrm{e}+}\right)\right)$ and integrating we get the positron rate required to impart the same level of KERMA as the ORTEC study to be

$$
\int \frac{\mathrm{d} N_{e+}^{\mathrm{mu} 2 \mathrm{e}}\left(E_{e+}\right)}{\mathrm{d} E_{e+}} \mathrm{d} E_{e+}=1.3423 \times 10^{13} \text { positrons }
$$


With a rate of 17097 (e+/e-)/ $\mathrm{cm}^{2} / \mathrm{s}$ it would take approximately 24 years 11 months before the HPGe crystal would require annealing. This is a significant increase to the rate obtained from the previous photon study and the detector would definitely need to be annealed much more regularly than this.
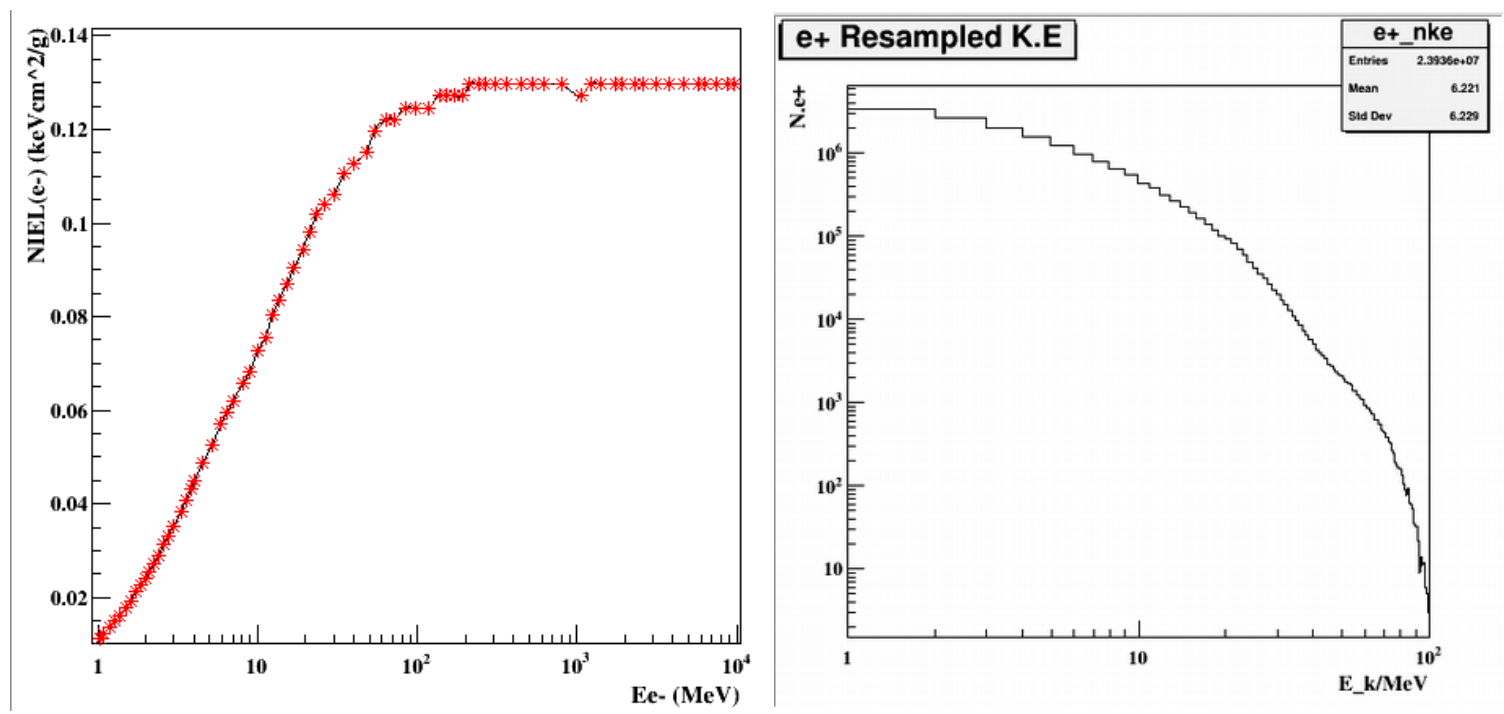

(a) Extracted NIEL for electrons/positrons (b) Kinetic energy spectrum for resampled from Figure 5.8 used in the calculations. positron data.

Figure 5.18: Positron extraction and data used for KERMA calculation.

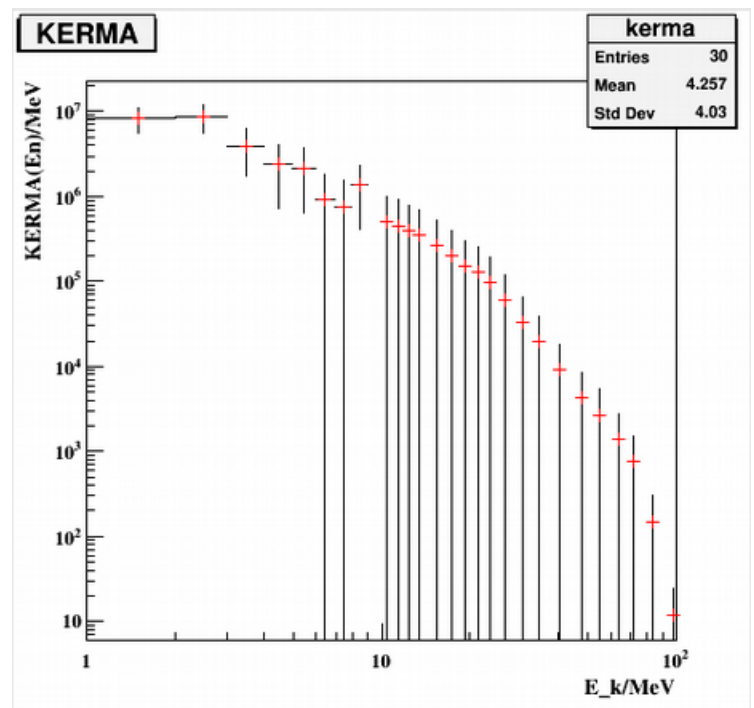

Figure 5.19: KERMA distribution as a function of positron energy for Mu2e. 


\section{Chapter 6}

\section{Conclusion}

This thesis has outlined the theoretical framework in which CLFV effects can appear in $\mu \rightarrow e$ transitions within and beyond the Standard Model. It has outlined prior limits set by CLFV experiments and has covered a detailed account of the Mu2e experiment and how it aims to improve the current limits on the $R_{\mu e}$ ratio. Finally it has offered a range of methods to calculate estimations for radiation damage to the STM, which is crucial for measuring the rate of stopped muons. Methods for establishing radiation damage of HPGe crystals are, in general, limited. This dissertation offers assessment of radiation damage caused by the harsh radiation environment of the STM beamline in Mu2e including that of fast neutrons and pair-produced positrons caused by the high energy gamma flash.

By extracting the NIEL distribution for incident particles and, by extending the KERMA parameter to the case of HPGe as a function of energy using data from the ORTEC study, allowed for an estimation of the neutron rate that would cause substantial damage to the HPGe crystals. Utilising the Geant4 based, Mu2e Offline software, by producing simulations based on input files and by resampling the data an estimation of the time before the crystal would need annealing could be obtained. It was found that in the case of neutrons the time before annealing was estimated to be 75 and 78 months and in the case of pair-produced positrons the time before annealing was estimated to be $\tau \sim 24$ years, 11 months, which shows an increase from the estimates from [64].

It was then established by generating neutrons that the Spot-Size collimator was ef-

fective at shielding the detector for fast neutrons $(\mathcal{O}(1 \mathrm{MeV}))$; however, the neutrons 
with energy in the extreme limits from the data $(\mathcal{O}(50-60 \mathrm{MeV}))$ passed through at a rate of $27.12 \%$. It can be concluded that the damage caused by photons via ionization damage, as well as the damage caused by pair-produced positrons, is small compared to the damage caused by neutrons. The damage caused by neutrons in the updated geometry shows a $\sim 4.6 \times$ improvement over the 2016 data showing the progress of design specifications to protect the STM from radiation damage, ensuring optimal performance. Due to the uncertainties in the resampling, the geometrical considerations, loss of solid angle and data extraction, as well as the rate for the very fast neutrons on the HPGe, being much greater, further research must be conducted to fully understand the damage caused by neutrons. 


\section{Bibliography}

[1] Carlson, J.F., and Oppenheimer, J.R., "On Multiplicative Showers", Physical Review, 51 (1936). pp.220-221.

[2] Neddermeyer, S.H., and Anderson, C.D., "Note on the Nature of Cosmic-Ray Particles", Physical Review, 51 (1937), pp.884-886.

[3] Street, J.C., Stevenson, E.C., "Penetrating Corpuscular Component of the Cosmic Radiation", Physical Review, 51 (1937), p.1005.

[4] Bellerive, A., Klein, J., McDonald, A., Noble, A., and Poon, A., "The Sudbury Neutrino Observatory", Nucl.Phys.B908,30(2016).

[5] van der Schaaf, A., "Forbidden Muon Decays", Prog.Part.Nucl.Phys.31(1993)1.

[6] Baldini, A., et al., "A submission to the 2020 update of the European Strategy for Particle Physics on behalf of the COMET, MEG, Mu2e and Mu3e collaborations", arXiv:1812.06540 [hep-ex].

[7] Bhattacharya, B., Morgan, R., Osborne, J., Petrov, A.A., "Studies of lepton flavor violation at the LHC", Physics Letters B, vol. 785(2018), 165-170, https://doi.org/10.1016/j.physletb.2018.08.037 (http://www.sciencedirect.com/science/article/pii/S0370269318306506)

[8] Cei, F., and Nicolo, D., "Lepton Flavour Violation Experiments", Advances in High Energy Physics, vol. 2014, 282915, 2014. doi:10.1155/2014/282915.

[9] Baldini, A.M., et al., "Search for the lepton flavour violating decay $\mu^{+} \rightarrow e^{+} \gamma$ with the full dataset of the MEG experiment", Eur.Phys.J C(2016) 76:434., doi:10.1140/epjc/s10052-016-4271-x 
[10] Bertl, W., et al., "A search for $\mu \rightarrow e$ conversion in muonic gold", Eur.Phys.J. C47, 337(2006), doi:10.1140/epjc/s2006-02582-x.

[11] Dohmen, C., et al., "Test of lepton flavor conservation in $\mu \rightarrow e$ conversion on titanium", Phys. Lett., B317 (1993), p. 631, doi:10.1016/0370-2693(93)91383-X

[12] Honecker, W. et al., "Improved limit on the branching ratio of $\mu \rightarrow e$ conversion on lead", Phys. Rev. Lett., 76 (1996), p.200, doi:10.1103/PhysRevLett.76.200

[13] Glashow, S.L, Nucl.Phys.22(1961) 579. Weinberg, S., Phys.Rev.Lett.19(1967)1264. Salam, A., Originally printed in "Svartholm: Elementary Particle Theory,Proceedings of the Nobel Symposium Held 1968 at Lerum, Sweden", Stockholm 1968, 367-377

[14] Nguyen, K., "Higgs Phenomenon, Renormalization Group Theory and Critical Phenomena Seminar Notes, LMU Munich, July 2009, https://www.theorie.physik.uni-muenchen.de/lsfrey/teaching/archiv/ sose_09/rng/higgs_mechanism.pdf

[15] Buchalla, G., "Introduction to The Standard Model", Lecture Notes in Theoretical Physics 6, LMU Munich, (2008)

[16] Calibbi, L., and Signorelli, G., "Charged Lepton Flavour Violation: An Experimental and Theoretical Introduction", Riv. Nuovo Cim.41 no. 2, (2018) 71174, arXiv:1709.00294 [hep-ph].

[17] Majorana, E., Nuovo Cimento 5 , 1937: p. 171184

[18] Bilenky, S.M., Giunti, C. and Grimus, W., "Phenomenology of neutrino oscillations", Prog. Part. Nucl. Phys.,43 (1999) 1 [hep-ph/9812360].

[19] Nagashima, Y., "Appendix C: Dirac Matrix and Gamma Matrix Traces", Elementary Particle Physics, 817-824, John Wiley \& Sons (2010), https: //onlinelibrary.wiley.com/doi/abs/10.1002/9783527630097.app3

[20] Wilczek, F., "Majorana returns". Nat Phys, 2009. 5(9): p. 614-618.

[21] Goldstone, J., Il Nuovo Cimento 19 (1961) 154 
[22] Zuber, K., "Double Beta Decay", Contemp. Phys. 45 (2004) 491-502

[23] Petcov, S., Shindou, T., and Takanishi, Y., "Majorana CP-violating phases, RG running of neutrino mixing parameters and charged lepton flavor violating decays", Nucl.Phys.B738 (2006) 219242, arXiv:hep-ph/0508243 [hep-ph].

[24] Cheng, T.P., and Li, L.F., "Gauge Theory Of Elementary Particle Physics", (OxfordScience Publications), 1984.

[25] Akhmedov, E.Kh., Branco, G.C., and Rebelo, M.N, "Seesaw mechanism and structure of neutrino mass matrix", hep-ph/9911364

[26] Fogliet, G.L, et al., "Global analysis of neutrino masses, mixings and phases: entering the era of leptonic CP violation searches", Phys. Rev. D86:013012, 2012.

[27] Cheng, T.P., and Li, L. (Dec 1980). " $\mu \rightarrow e \gamma$ in theories with Dirac and Majorana neutrino-mass terms". Physical Review Letters, 45(24), 1908-1911.

[28] Gell-Mann, M., Slansky, R., and Stephenson, G., un-published; Barger, V., Langacker, P., Leveille, J.P., and Pakvasa, S., University of Wisconsin Report No.COO-881-149, 1980 (unpublished); Cheng, T.P., and Li, L.F., "Gauge Theory Of Elementary Particle Physics" Ref.1

[29] Gell-Mann, M., Ramond, P., and Slansky, R., "in Supergravity", edited by Freedman, D.Z., Nieuwenhuizen, P.van, (North-Holland, Amsterdam, 1979).

[30] Bartolotta, A., and Ramsey-Musolf, M.J., "Coherent $\mu \rightarrow e$ conversion at nextto-leading order", Phys. Rev. C 98, 015208 (July 2018)

[31] Cirigliano, V., Kitano, R., Okada, Y., and Tuzon, P., "Model discriminating power of $\mu \rightarrow e$ conversion in nuclei", Phys. Rev. D80 (Jul, 2009) 013002.http://link.aps.org/doi/10.1103/PhysRevD.80.01300

[32] Shifman, M., Vainshtein, A., and Zakharov, V., "Remarks on higgs-boson interactions with nucleons", Physics Letters B78 no.4, (1978) 443-446. http: //www.sciencedirect.com/science/article/pii/0370269378904811. 
[33] De Gouvea, A.L., and Vogel, P., (2013). "Lepton flavor and number conservation, and physics beyond the standard model", Progress in Particle and Nuclear Physics, 71, 75-92. https://doi.org/10.1016/j.ppnp.2013.03.006

[34] Haber, H.E., and Haskins, L.S., "Supersymmetric Theory and Models", Anticipating the Next Discoveries in Particle Physics (2018), arXiv:1712.05926

[35] Ding, J.N., Qin, Q., and Fu-Sheng Y., "Heavy Neutrino Searches at Future Z-Factories", The European Physical Journal C 79.9 (2019), arXiv:1903.02570

[36] Branco, G.C., et al., "Theory and Phenomenology of Two-Higgs-Doublet Models", Physics Reports 516.1-2 (2012): 1102, arXiv:1106.0034

[37] Sannino, F., Strumia, A., Tesi A., and Vigiani, E., "Fundamental partial compositeness", JHEP11(2016): 029, arXiv:1607.01659

[38] Buchmuller, W., Ruckl, R., Wyler, D., "Leptoquarks in Lepton Quark collisions", Phys. Lett. B191 (1987): 442448, https://doi.org/10. 1016/S0370-2693(99)00014-3, https://doi.org/10.1016/0370-2693(87) 90637-X. [Erratum: Phys. Lett.B448,320(1999)]

[39] Nachtmann, O., Nagel, F., and Pospischil, M., "Anomalous Gauge-Boson Couplings and the Higgs-Boson Mass", The European Physical Journal C 42.2 (2005): 139161, arXiv:hep-ph/0404006

[40] Raidal, M. et al., "Flavour physics of leptons and dipole moments", Eur. Phys. J.C57(2008)13182, arXiv:0801.1826

[41] Bartoszek, L. et al., "Mu2e Technical Design Report", FERMILAB-TM-2594, FERMILAB-DESIGN-2014-01, arXiv:1501.05241 (2014)

[42] Marciano, W.J. et al, "Charged Lepton Flavor Violation Experiments", Annual Review of Nuclear and Particle Science, 58, 1, 315-341, 200

[43] Bonventre, R., "Searching for muon to electron conversion: The Mu2e experiment at Fermilab", SciPostPhysics Proceedings(2019) 038.

[44] Suzuki et al., Phys.Rev.C35, 2212 (1987) 
[45] Bernstein, R., "Requirements Document for Mu2e Tracker", Mu2e-doc-732.

[46] Lucchesi, L.(2017), "Design, thermal analysis and validation test of the Mu2e electromagnetic calorimeter cooling system at Fermilab" (Masters Thesis), Universit di Pisa, Mu2e-doc-15000-v1

[47] Miller, J., Bernstein, R., et al, "Requirements \& Specifications for WBS 5.6 Muon Stopping Target Monitor", Mu2e-doc-1438.

[48] Bernstein, R., "Stopping Target Monitor Rates", Mu2e-doc-4010.

[49] Palladino, A., Quirk,J., Miller,J., "Mu2e Stopping-Target Monitor baseline design", Mu2e-doc-6453

[50] Quirk, J., "AlCap Photon Study Update, Mu2e-doc-5126; Quirk,J., Mu2e-doc6192; "AlCap Update: Status and Plans, Quirk,J., "AlCap Photon Spectra, Mu2e-doc-6725.

[51] $\pi$ E5 Beam Line, The Paul Scherrer Institut (PSI), https://www.psi.ch/en/ sbl/pie5-beamline

[52] Mu2e Collaboration, "Requirements \& Specifications for WBS 5.8 - Muon Beam Stop", Mu2e-doc-1351.

[53] Palladino, A., "Stopping-Target Monitor Alignment", Mu2e-doc-731.

[54] Glenzinski, D., and Miller,J., "Mu2e Science Requirements, Mu2e-doc-4381v6.

[55] Knoll, G.F., "Radiation Detection and Measurement"., New York: John Wiley \& Sons, Inc. Second Edition(1989).

[56] ORTEC ${ }^{\circledR}$, "GMX Series Coaxial HPGe Detector Product Configuration Guide", https://www.ortec-online.com/-/media/ametekortec/brochures/ gamma-x.pdf

[57] ORTEC $^{\circledR}$, "Overview of Semiconductor Photon Detectors", https://www.ortec-online.com/-/media/ametekortec/other/ overview-of-semiconductor-photon-detectors.pdf 
[58] IEEE, "Standard Test Procedures for Germanium Gamma-Ray Detectors", in IEEE Std 325-1996, vol., no., pp.0_1-, 1997.

[59] Pehl, H., et al., "Accurate Determination of the Ionization Energy in Semiconductor Detectors, Nucl. Instr. and Meth.59(1988)45.

[60] Shah, K.S., Glodo,J., Klugerman,M., Moses,W.W., Derenzo,S., and Weber,M.J.(2004). "LaBr3:Ce Scintillators for Gamma-Ray Spectroscopy". Nuclear Science, IEEE Transactions on. 50. 2410 - 2413. 10.1109/TNS.2003.820614.

[61] ORTEC ${ }^{\circledR}$, "Lanthanum Bromide Scintillation Detectors", https://www. ortec-online.com/-/media/ametekortec/brochures/lanthanum.pdf

[62] Palladino, A., "Stopping Target Monitor design: using a fast scintillator to detect the $1.809 \mathrm{MeV}$ gamma for mu2e normalization", Mu2e-doc-5168

[63] Tishchenko, V., et al., "Gamma-ray Irradiation Test of a High Purity Germanium Detector", Mu2e-doc-7042

[64] Palladino, A., "Mu2e Stopping-Target Monitor: Radiation Damage", Mu2e-doc6415

[65] Sutton, A.,(2019). "Displacement Damage and Ionization Effects in Advanced Silicon-Germanium Heterojunction Bipolar Transistors", PhD thesis, Georgia Institute of Technology, 2005.

[66] Kinchin, G.H., Pease, R.S., "The Displacement of Atoms in Solids by Radiation", Rep. Progr. Phys., 18, 1 (1955).

[67] Norgett, M.J., Robinson, M.T., Torrens,I.M., "A Proposed Method of Calculating Displacement Dose Rates", Nucl. Eng Des., 33, 50 (1975).

[68] Fasso, A., Ferrari,A., Smirnov, G., Sommerer,F., Vlachoudis,V.,(2011). "FLUKA Realistic Modeling of Radiation Induced Damage". Progress in Nuclear Science and Technology. 2. 769-775.

[69] Dale,C.J., Marshall,P.W., Summers,G.P., Wolicki,E.A., Burke, E.A., "Displacement Damage Equivalent to Dose in Silicon Devices", Appl. Phys. Lett., 54 (1989), pp.451-453 
[70] Akkerman, A., Barak, J., Chadwick, M., Levinson, J., Murat, M., and Lifshitz, Y., "Updated NIEL calculations for estimating the damage induced by particles and $\gamma$-rays in Si and GaAs", Radiat. Phys. Chem.62(2001) 301-310

[71] ASTM E722-14, "Standard Practice for Characterizing Neutron Fluence Spectra in Terms of an Equivalent Monoenergetic Neutron Fluence for RadiationHardness Testing of Electronics", ASTM International, West Conshohocken, PA, 2014

[72] Green, C., et al (2012), "The Art Framework", J. Phys.: Conf. Ser.396 022020, https://doi.org/10.1088/1742-6596/396/2/022020

[73] Miller, J., Tran, M., and Wang, Y., "STM simulation update', Mu2e-doc-16136v1

[74] Pehl, R.H., Madden, N.W., Elliott, J.H., Raudorf, T.W., Trammell, R.C., and Darken, L.S., "Radiation Damage Resistance of Reverse Electrode GE Coaxial Detectors," in IEEE Transactions on Nuclear Science, vol. 26, no. 1, pp. 321-323, Feb. 1979 .

[75] Rohatgi, A., WebPlotDigitizer, version 4.2, (April 2019) https://automeris. io/WebPlotDigitizer 